\title{
Erik Garbacik
}

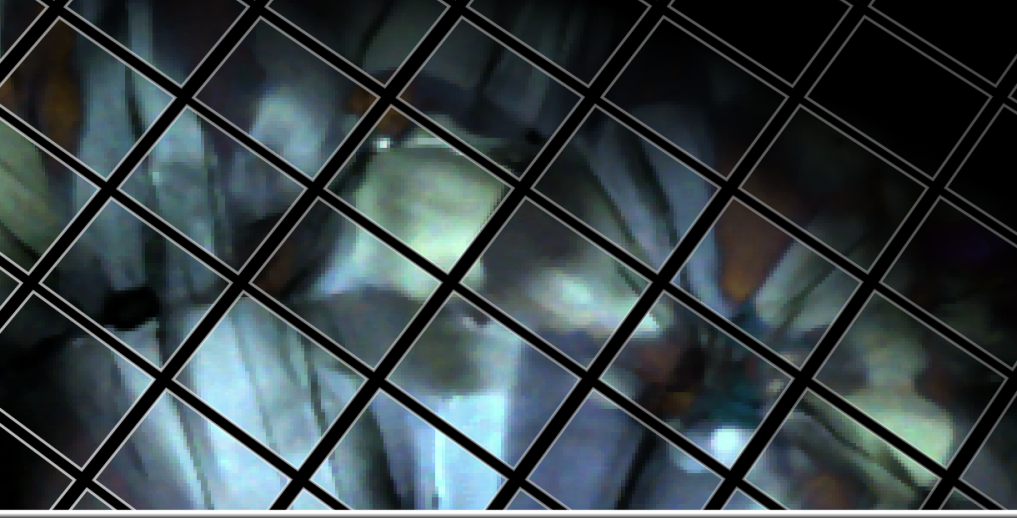

\section{Contrast in coherent Raman scattering microscopy}

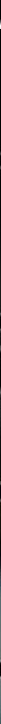




\section{CONTRAST IN COHERENT \\ RAMAN SCATTERING MICROSCOPY}


Thesis committee members

Supervisor

Assistant supervisor

Chair and secretary

Invited members
Prof. dr. J. L. Herek

Dr. ir. H. L. Offerhaus

Prof. dr. K. J. Boller

Prof. dr. W. L. Barnes

Prof. dr. J. F. de Boer

Prof. dr. J. R. Brandenberger

Dr. C. Otto

Prof. dr. W. S. Warren
Universiteit Twente

Universiteit Twente

Universiteit Twente

Universiteit Twente

Exeter University

Vrije Universiteit Amsterdam

Lawrence University

Universiteit Twente

Duke University

This work was carried out at the Optical Sciences group, which is a part of:

Department of Science and Technology

and $\mathrm{MESA}^{+}$Institute for Nanotechnology,

University of Twente, P.O. Box 217,

7500 AE Enschede, The Netherlands.

Financial support was provided by a VICI grant from the CW section of the Nederlandse Wetenschappelijk Organisatie (NWO) to Prof. Jennifer L. Herek. Additional funding was provided by FOM, NWO, and STW.

ISBN: 978-90-365-3674-5

Copyright (C)2014 by Erik Garbacik

All rights reserved. No part of the material protected by this copyright notice may be reproduced or utilized in any form or by any means, electronic or mechanical, including photocopying, recording or by any information storage and retrieval system, without the prior permission of the author. 


\section{CONTRAST IN COHERENT RAMAN SCATTERING MICROSCOPY}

\section{DISSERTATION}

to obtain

the degree of Doctor at the University of Twente, on the authority of the Rector Magnificus,

Prof. dr. H. Brinksma,

on account of the decision of the graduation committee,

to be publicly defended,

on Friday, May 23, 2014 at 16:45

by

Erik Thomas Garbacik

born on May 1, 1986

in Mountain View, CA, USA 
This manuscript has been approved by:

Supervisor: Prof. dr. J. L. Herek

Assistant supervisor: Dr. ir. Herman L. Offerhaus 


\section{Contents}

1. Introduction $\quad \mathbf{1}$

1.1. Concepts in light microscopy . . . . . . . . . . . . . . 1

1.2. Contrast mechanisms . . . . . . . . . . . . . . . . . 3

1.3. Coherent Raman scattering ............. . . 5

1.4. Overview .......................... 9

2. Hyperspectral CARS microscopy 11

2.1. Classical theory of coherent Raman scattering . . . . . . . . . . 13

2.1.1. Nonlinear optics . . . . . . . . . . . . . . . . 13

2.1.2. Coherent anti-Stokes Raman scattering . . . . . . . . 14

2.2. Experimental details . . . . . . . . . . . . . . . 17

2.2.1. Optical setup . . . . . . . . . . . . . . 17

2.2.2. Acquisition and synchronization . . . . . . . . . . . 19

2.2.3. Hyperspectral projection . . . . . . . . . . . . 21

2.3. Results . . . . . . . . . . . . . . . . . 23

2.3.1. Rapid identification of heterogeneous mixture components 23

2.3.2. In situ analysis of crystal polymorphs . . . . . . . . . . 27

2.3.3. In planta imaging of $\Delta^{9}$-tetrahydrocannabinol . . . . . . 37

3. Vibrational phase contrast CARS $\quad 47$

3.1. Theory . . . . . . . . . . . . . . . 47

3.1.1. The molecular rollercoaster . . . . . . . . . . . . . 47

3.1.2. Heterodyne phase detection . . . . . . . . . . . . . . 49

3.1.3. Homodyne phase detection . . . . . . . . . . . 50

3.2. Experimental apparatus . . . . . . . . . . . . 56

3.2.1. Initial version . . . . . . . . . . . . . . . . 57

3.2.2. Current version . . . . . . . . . . . . . 57

3.3. Results . . . . . . . . . . . . . . . 60

3.3.1. Epi-detection . . . . . . . . . . . . 60

3.3.2. Spectral unmixing of complex data . . . . . . . . . . 61

3.3.3. High-speed phase-sensitive imaging . . . . . . . . . . . 73

3.3.4. Dual-frequency VPC-CARS . . . . . . . . . . 73 
4. Vibrational molecular interferometry $\mathbf{7 9}$

4.1. Theory . . . . . . . . . . . . . . . . . . . 79

4.2. Experimental setup . . . . . . . . . . . . . 85

4.3. Results . . . . . . . . . . . . . . . 86

4.3.1. Resonant vibrational spectroscopy . . . . . . . . . 86

4.3.2. Resonant vibrational microscopy . . . . . . . . . . . . 87

4.3.3. Electrovibrational spectroscopy . . . . . . . . . . . . 89

$\begin{array}{ll}\text { 5. Conclusions and outlook } & 91\end{array}$

6. Acknowledgments $\quad 95$

$\begin{array}{lr}\text { A. Noise in VPC-CARS measurements } & 109\end{array}$

$\begin{array}{ll}\text { B. Summary } & 113\end{array}$

$\begin{array}{ll}\text { C. Samenvatting } & 117\end{array}$

$\begin{array}{ll}\text { D. List of publications } & 121\end{array}$ 


\section{Introduction}

Contrast is arguably the single most important concept in imaging. Defined most broadly as the difference between objects or entities, contrast can take many different forms. In visual terms, the most striking contrasts are those of luminance and hue, from which entire fields of study and hobby have been created. Luminance is a measure of the total amount of light radiated from an object. Strong luminance contrast can be observed by gazing at the bright full Moon in the clear night sky; the Moon's bright white disc stands out starkly from the dark black background of space. Hue, on the other hand, represents the color of the light being measured. A field of flowering tulips, each variety displaying its own particular color, has exceptional contrast due to the spectrum of different hues, even when the luminance is roughly uniform. Representative images of these examples of hue and luminance contrast are shown in Figs. 1.1. The remarkable ranges of these two parameters span orders of magnitudes of space; the information that can be extracted from every image, from those covering very small areas (less than the width of a human hair) to the very large (billions of light years), depends critically on contrast.

\subsection{Concepts in light microscopy}

The light microscope is a device that magnifies small objects using radiation in the near-ultraviolet, visible, and/or near-infrared regions of the electromagnetic spectrum. Ranging from simple and ubiquitous upright laboratory inspection microscopes to specialized multi-million dollar laser-based scanning systems, the usefulness of the microscope is subject to the contrast of the data it produces. Contrast itself is not a fixed parameter. For example, a typical mammalian epithelial cell is nearly transparent. An image of one of these cells taken with an ordinary light microscope allows the user to determine its shape and size, and perhaps identify a few of the more distinctive intracellular structures, but smaller and finer details are lost. The introduction of dye that stains 

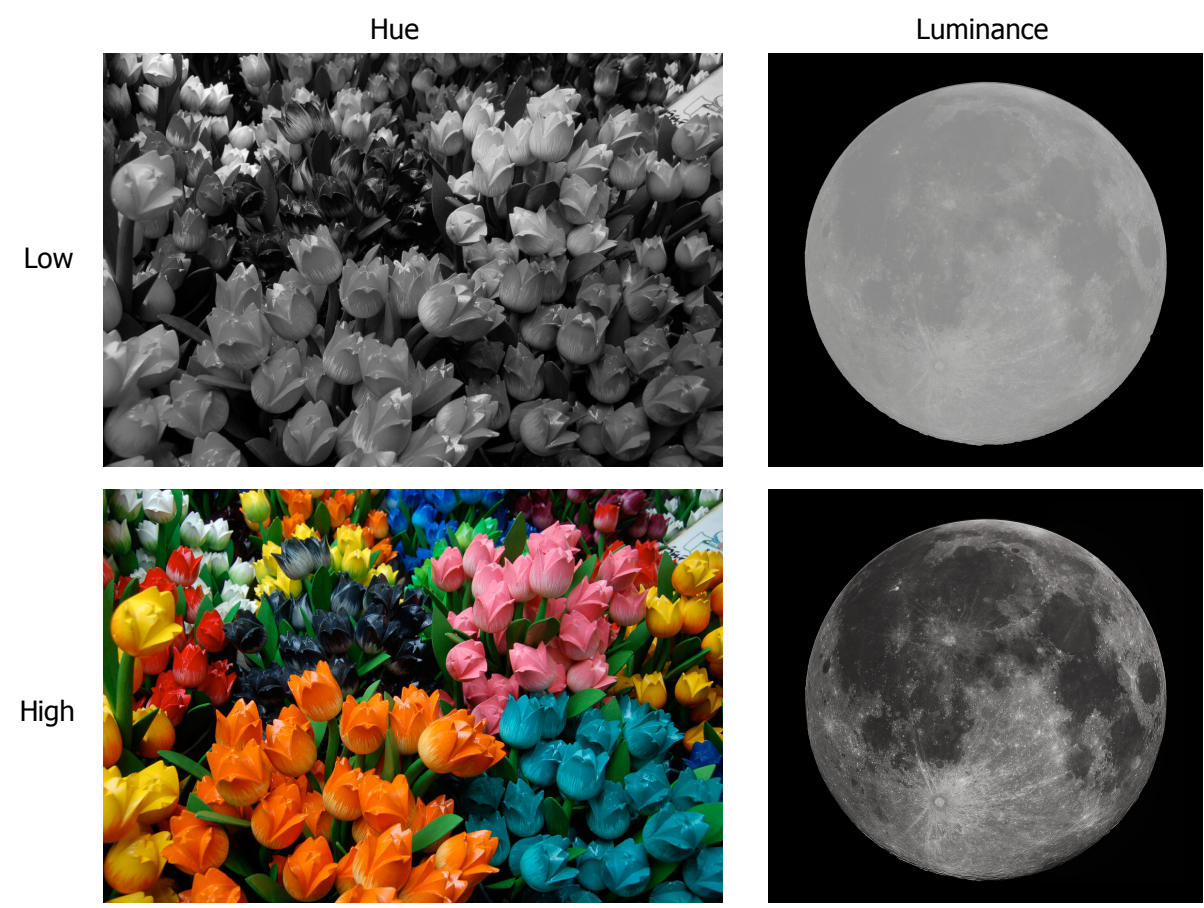

Figure 1.1. Representative images containing weak hue and luminance contrast (top row), and the same images with high contrast (bottom row). Tulip image courtesy of Bill Schleicher.

a certain cell organelles will dramatically increase the overall selectivity of the image for that particular organelle. The simultaneous application of multiple dyes, each targeting a separate organelle, creates the specificity of the image. A highly specific image can - in theory-be processed to select the individual organelles.

Both specificity and selectivity are somewhat qualitative terms that describe the contrast of the data in an image, and each can be enhanced via a variety of techniques at the sample preparation, image acquisition, and processing levels. However, there are two crucial parameters that can only be improved in very specific circumstances, and even then only with difficulty. The resolution of an optical system describes its ability to distinguish between two features in the sample. In conventional light microscopy the spatial resolution is limited by diffraction, which sets a lower limit on the distance between two objects to be individually distinguishable[1]. This minimum separation is typically 

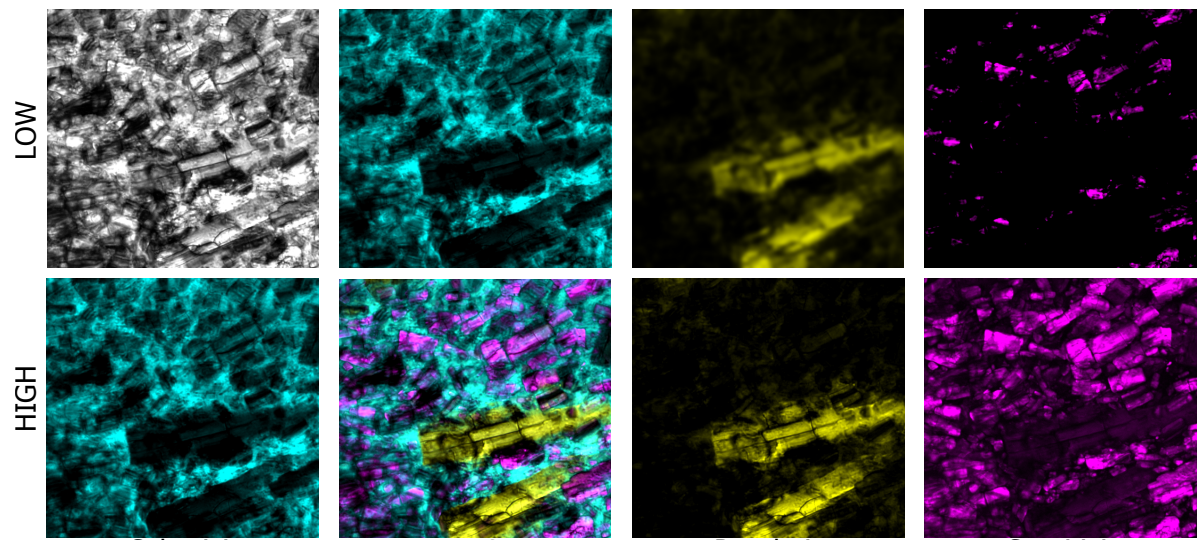

Specificity

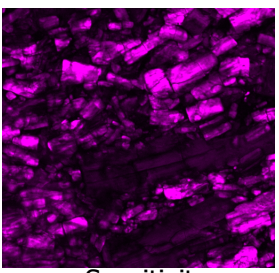

Resolution

Sensitivity

Figure 1.2. Visual definitions of concepts in light microscopy. From left to right: high specificity allows us to see that there are three different compounds in the sample. We can then select one of the compounds to visualize is more precisely. High sensitivity enables us to see fainter details. Finally, if the resolution is not sufficient the images experience blurring, masking fine structures.

about half the wavelength of the light used to perform the imaging with highmagnification objectives. The spectral resolution of a system is defined by the light source and/or detector, and can be almost limitlessly small.

In most optical experiments the data arrive in the form of photons. The sensitivity of the experiment is related to the number of photons required to register a signal. Detectors with excellent intrinsic noise characteristics can discriminate single photons in the absence of a significant optical background. In general, the sensitivity on the detection side of an optical microscope is fixed, and sensitivity can only be improved by increasing the amount of power on the excitation side. However, this approach is not without its drawbacks: high-power lasers are easily capable of damaging fragile samples!

\subsection{Contrast mechanisms}

In the vast majority of images, the three main optical processes by which contrast is created are absorption, emission, and scattering. Absorption and scattering are universal contrast mechanisms. Colors that we see around us are the result of a combination of both processes; the dark green color of leaves on a tree results from the strong absorption of red and blue light and the scattering 
of green light. Likewise, black objects tend to absorb strongly while white objects scatter efficiently across the visible spectrum. Since both absorption and scattering are natural contrast mechanisms, it is easy to see how they were first exploited in early microscopes for bright-field[2] and dark-field[3] configurations, respectively. In a similar vein the use of dyes to enhance image contrast by modulating the absorption of light across the sample is no surprise. However, some of these dyes are toxic, carcinogenic, affect the behavior of the sample, or are otherwise unsuitable for use in in vitro or in vivo experiments.

A major breakthrough in microscopy was the discovery of phase contrast microscopy by Fritz Zernike in 1942[4]. The wavefronts of light passing through a sample are delayed as a function of both the refractive index and the thickness of the sample. If different regions of the sample contain variations in either of these two parameters, then the wavefronts of the light passing through these regions are delayed relative to each other. In normal bright-field microscopy these phase delays cannot be detected, but the Zernike phase contrast microscope translates phase differences into intensities. Phase contrast microscopy provides rich information about structural morphology, but is limited to thin samples and cannot reveal details about their chemical compositions.

In 1923, Adolf Smekal predicted that a sample in the path of monochromatic light would spontaneously scatter new wavelengths of light corresponding to the vibrations of its constituent molecules[5]. This effect was observed a few years later by Sir C. V. Raman[6], who would receive the Nobel Prize in Physics and give his name to the process: spontaneous Raman scattering. In a simplified picture, when a photon with energy $\hbar \omega_{p}$ strikes a molecule in its ground state some of the energy of that photon can be transferred into a vibrational mode with energy quantum $\hbar \Omega$. The remaining energy will be scattered away from the molecule as a photon with energy $\hbar \omega_{s}$. Alternatively, light incident on a molecule already in a vibrationally excited state can be scattered with a shorter wavelength. These two processes are more specifically known as Stokes and anti-Stokes Raman scattering, respectively. The difference in optical frequencies between the incoming and outgoing light $\left|\omega_{p}-\omega_{s}\right|=\Omega$ is identical to the frequency of the excited molecular vibration. A full map of the vibrations in a molecule can be recorded by measuring the light scattered from the sample at all wavelengths away from than that of the incident field. 


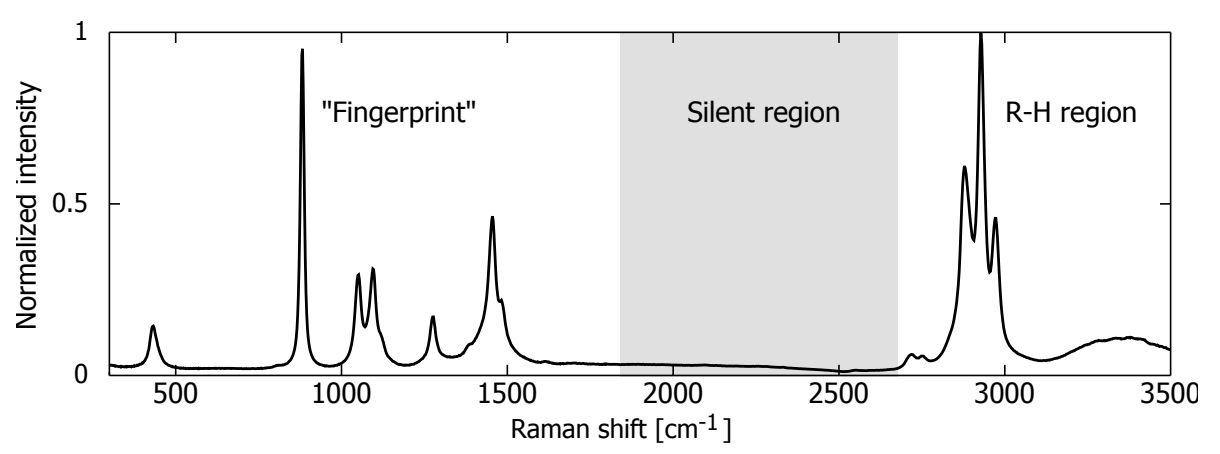

Figure 1.3. Spontaneous Stokes Raman scattering spectrum of ethanol. The peaks correspond to specific vibrational motions of the ethanol molecule. The peaks at frequencies below $1800 \mathrm{~cm}^{-1}$ are considered to be in the highly specific "fingerprint" region of the spectrum. Few molecular bonds oscillate in the silent region at frequencies between $1800 \mathrm{~cm}^{-1}$ and $2600 \mathrm{~cm}^{-1}$. The high-frequency region above $2600 \mathrm{~cm}^{-1}$ is home to a variety of resonances of carbon-hydrogen groups.

\subsection{Coherent Raman scattering}

Spontaneous Raman scattering is useful for determining the chemical makeup of a sample. Its primary disadvantage compared to other microscopy techniques is its lack of speed. The scattering process is omnidirectional and infrequent; only one photon in every or so trillion will be Raman scattered[7], and of these scattered photons fewer than $10 \%$ will be detected. Long integration times are therefore required to collect enough photons to build up a spectrum with sufficient contrast[8].

An alternative to spontaneous Raman scattering is a class of techniques collectively referred to as coherent Raman scattering (CRS)[9]. Rather than using a single monochromatic incident wave and collecting the spontaneously scattered light, CRS utilizes two or more synchronized lasers at different frequencies to actively excite the molecular vibrations. When these lasers are tuned so that the difference in their frequencies corresponds exactly to the natural frequency of a vibrational mode of the sample the molecule will be resonantly driven into oscillation. The resulting vibration can then be probed by a third laser beam; if the resulting emission is blue-shifted relative to the probe wavelength, then the entire process is referred to as coherent anti-Stokes Raman scattering, or CARS for short[10].

A CARS-capable microscope can be used to readily distinguish between ma- 

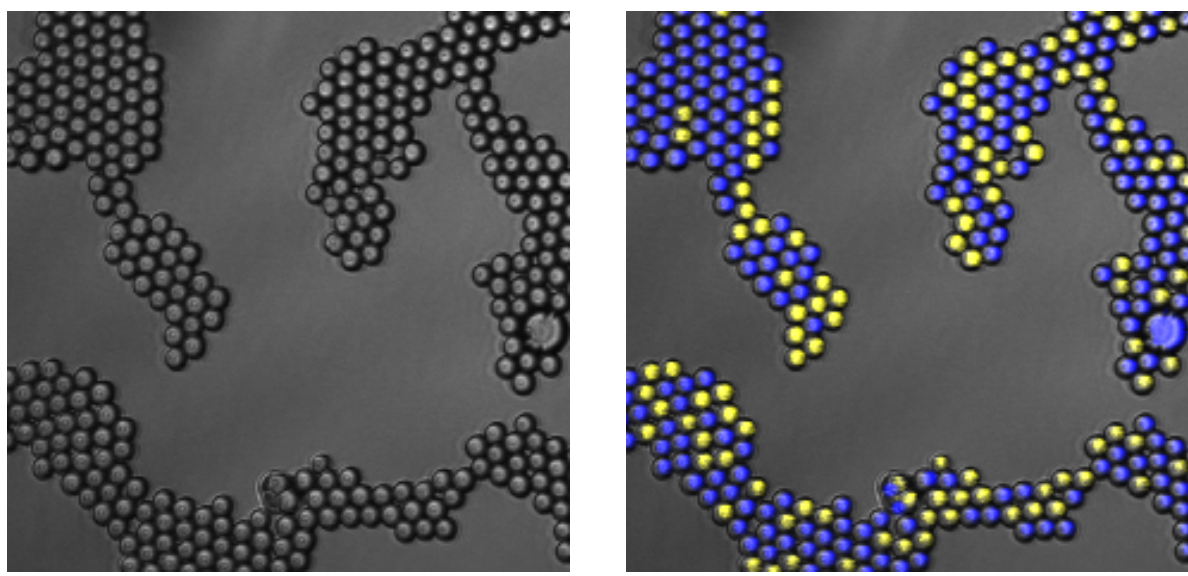

Figure 1.4. (left) Two types of transparent plastic beads, made of polystyrene (PS) and polymethylmethacrylate (PMMA), are indistinguishable under white light illumination. (right) Coherent anti-Stokes Raman scattering (CARS) microscopy allows easy discrimination between the two, with PS shown in blue and PMMA in yellow.

terials that appear identical under white-light illumination. The classic example is a distribution of two types of tiny plastic beads, as shown in Fig. 1.4. It is impossible to distinguish the two plastics with a traditional microscope, but tuning in to the characteristic vibrations of each plastic enables immediate and unambiguous identification. Due to the coherent nature of the optical process, CARS can yield orders of magnitude more signal than spontaneous Raman scattering[11], though with a few drawbacks. Current technology limits high-speed microscopy to a single vibrational frequency at a time, and a persistent nonresonant background is always present that can significantly decrease the spectral and spatial contrast of an image, particularly for weakly resonant samples.

Although the imaging component of a CARS microscope is limited to probing a single vibrational frequency at a time, certain laser systems can be tuned over wide frequency ranges with relative ease[12]. Coupling a fast imaging system with a sweeping narrowband laser source allows for hyperspectral imaging, wherein many individual images are recorded in sequence at slightly different vibrational frequencies. The resulting data is represented as a datacube, with two sides of the cube (height and width) representing the horizontal and vertical dimensions of the image and one side of the cube (depth) containing the 


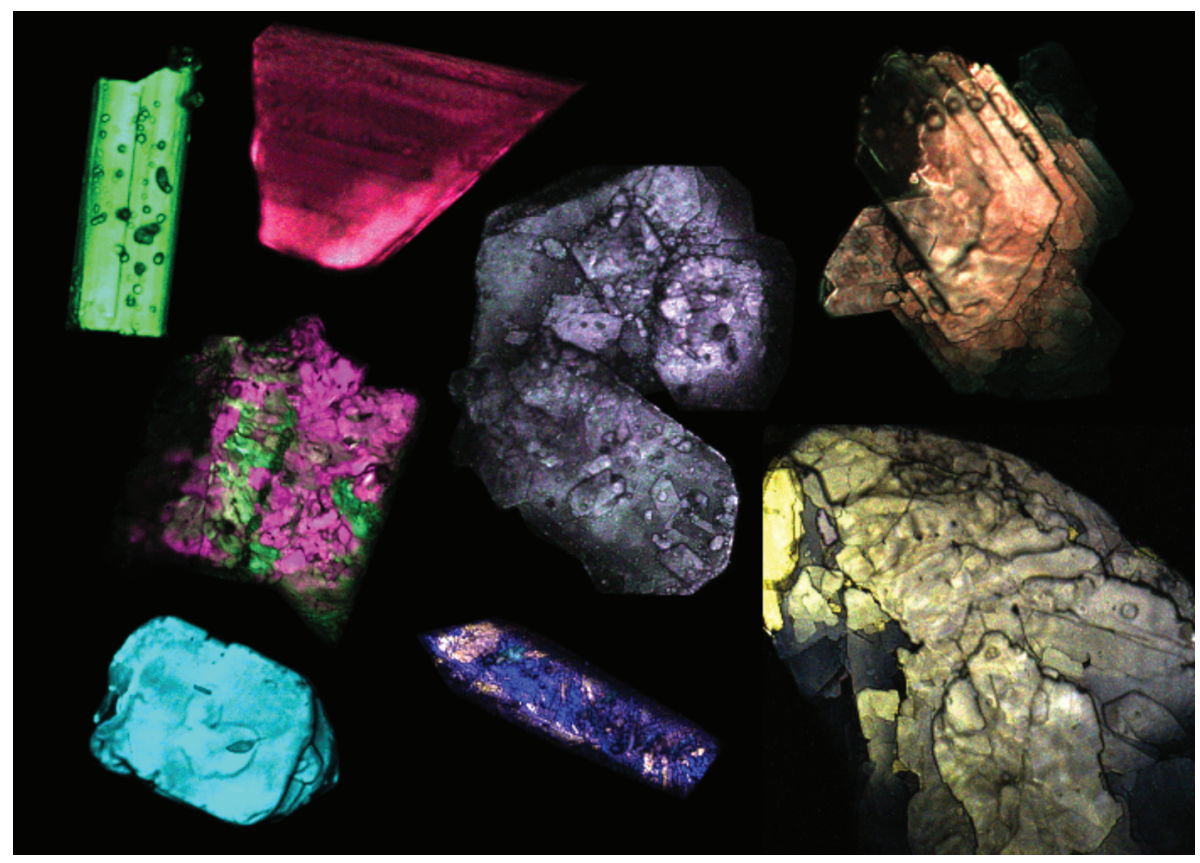

Figure 1.5. Amino acid crystals imaged with hyperspectral CARS microscopy. The colors represent different vibrational spectra. Clockwise from top-right: isoleucine, leucine, arginine, methionine, proline, glutamine, threonine, and tryptophan.

frequency information. We have developed a system to acquire and process this datacube so that each vibrational frequency is assigned a unique color; when all of the individual colored images are then added together, each material within the image will appear with its own hue. Shown in Fig. 1.5 are a group of nine different amino acids imaged with this technique. One of the most powerful aspects of this technique is that it is sensitive to not only differences of intra-molecular structure, but differences in inter-molecular structure as well. The orientations of molecules within a crystal lattice can drastically change the vibrational spectrum of the sample. While it is a potent tool for quickly analyzing the distributions of compounds within a sample, hyperspectral CARS is still affected by the non-resonant background, and is not compatible with moving samples.

To overcome both of these drawbacks an extended heterodyne technique called vibrational phase contrast CARS was created. Whereas a traditional 
CARS measurement detects the intensity of the light emitted by the sample a heterodyne measurement mixes the emitted light with a stable reference beam, known as a local oscillator. Although technically complicated, the advantages of heterodyne CARS are numerous. Building on the experiments of Potma et al.[13], Martin Jurna and his co-workers built a system to monitor both the amplitudes and phases of the molecular oscillators within the sample[14]. This pair of measurements forms a vector in the complex plane, and the entire vibrational spectrum of a molecule traces out a continuous curve through complex space[15]. Previous work by Jurna and Garbacik et al.[16] shows that the representation of Raman-active resonances as a set of "molecular rollercoasters" is a powerful tool for quantitatively analyzing mixtures of multiple components, even when only a single vibrational frequency is probed. Advances in electronics, software, and hardware now enable us to acquire these data at high speed, in thick samples, and over wide frequency ranges.

Heterodyne CARS experiments such as VPC-CARS are explicitly interferometric; a phase-stable local oscillator is mixed with the anti-Stokes field at a detector to extract the amplitude and phase of the vibrational response. This approach is inherently flawed in that the local oscillator is singled out from the beginning, and experiments with the same experimental configuration where different pulses are detected cannot be readily compared. When heterodyne CARS is instead developed in a quantum mechanical framework the description of the field mixing is much more transparent[17]. Rather than monitoring amplitudes and phases, this new framework requires merely measuring the numbers of photons created and annihilated in the various fields. The contributions to the total photon numbers from the parametric and dissipative optical processes - those in which energy is either re-arranged between the field modes or deposited into the molecule, respectively - are trivial to extract from the resulting data. A further advantage discovered serendipitously within this framework is that optical processes such as resonant two-photon absorption, in which all fields experience net photon number change, can be monitored readily without any further additions to the experimental setup. We refer to this measurement technique as vibrational molecular interferometry to emphasize that the star of the show is the molecule, rather than the interference of fields at the detector as in traditional heterodyne CARS measurements. 


\subsection{Overview}

This thesis describes three recent developments that increase the contrast of coherent Raman scattering experiments, divided into their own chapters.

CHAPTER II is about the basic theoretical and experimental implementations of hyperspectral coherent anti-Stokes Raman scattering (CARS) in a spectrally narrowband setup. The thrust of this research was the creation of hyperspectral acquisition routines and analysis tools that enable the rapid, qualitative characterizations of samples in-line. We built a system that is capable of acquiring hyperspectral CARS data stacks in tens of seconds, then quickly processing the complicated three-dimensional data to yield a set of intuitive, two-dimensional images with high visual contrast[18]. This system has become the workhorse of the Optical Sciences CARS lab-it is routinely used as a first-stage diagnostic tool for new samples - and has found use in a number of applications, including the in situ analysis of the constituent components of pharmaceutical oral dosage forms[19]. The introduction of a modulation technique further enables the suppression of certain electronic background contributions, which allowed us to image the in planta distributions of the active compounds in Cannabis sativa L.[20]

CHAPTER III covers vibrational phase contrast (VPC)-CARS. The basic theory and initial experimental implementations of VPC-CARS were carried out by Jurna et al. $[14,21]$, and this work expands on both. On the theory side, we have expanded the mathematical framework of coherent Raman scattering to describe molecular systems where multiple resonances in different regions of the vibrational spectrum are probed simultaneously. Computational work based on this new model showed that two resonances in different vibrational manifolds can either mutually enhance or suppress the total vibrational signal. Experimentally, we have implemented an entirely new hardware architecture to increase the imaging speed of the system by over two orders of magnitude compared to its predecessor, as well as added capabilities for imaging in epi-detection when detection in transmission is not possible. Further, the hyperspectral scanning capabilities of the previous section are applied to VPC-CARS. Complex vibrational spectra are collected at every point within a sample, enabling analysis beyond what is possible with data obtained from other methods. This analysis includes the first application of a quantitative endmember extraction algorithm called SPICE (Sparsity Promoting Iterative Constrained Endmember detection)[22] on complex hyperspectral data. This algorithm attempts to determine the precise number of pure compounds that 
are present in a given sample based on a set of mixed data, as well as the spectra and relative proportions of those compounds within each pixel of the image. An initial analysis of the VPC-CARS data measured on quench-cooled mannitol, which is a highly complicated sample, has been very promising.

CHAPTER IV introduces a new paradigm regarding CRS measurements. Building on the theoretical groundwork laid down by Rahav and Mukamel[17] we have developed an experimental technique that casts heterodyne CARS in a fully quantum mechanical framework. The primary advantages of this new $v i$ brational molecular interferometric (VMI) approach are twofold. First, by not singling out one beam in a heterodyne CARS experiment as the "local oscillator" from the beginning, all fields are treated equally and hence the analysis of the interaction is much simpler. Casting the optical interactions in terms of parametric and dissipative components further simplifies the interpretation of the data, and facilitates the direct extraction of the purely resonant component of the nonlinear susceptibility $\chi^{(3)}$. Second, although not explicitly predicted by Rahav and Mukamel, two-photon resonant electronic transitions can be measured simultaneously. With our setup we have measured the purely resonant component of a molecular vibration in the presence of a large electronic two-photon fluorescent background that overwhelms a standard CARS measurement $[23,24]$. 


\section{CHAPTER 2}

\section{Hyperspectral CARS microscopy}

Coherent anti-Stokes Raman scattering (CARS) microscopy has gone through a number of iterations since the first demonstration of a non-collinear system by Duncan et al. in 1982[25]. Important advancements were the demonstration of collinear CARS microscopy in 1999 by Zumbusch et al.[26] and the combination of a collinear CARS microscope with a galvanometric laser-scanning head[27, 28 ] to allow imaging at speeds up to video rate[29]. Nearly a decade and a half after the first collinear CARS microscopy a tremendous number of additional techniques have been introduced. The effects of adjusting every property of the input fields have been well studied: a small sample includes polarization[30-37]; temporal overlap[38-42]; focusing geometry[10, 43-45]; phase modulation[13, 46-48]; spectral phase[49-52]; and frequency modulation[53, 54].

Generally speaking, CARS microscopy falls into two broad categories: narrowband CARS, where the frequency bandwidth of the driving lasers is on the order of a vibrational natural linewidth[55]; and broadband or multiplex CARS, where the spectral bandwidth of at least one of the driving lasers is significantly larger than a vibrational linewidth[56-65]. There are two primary experimental advantages to a narrowband approach. First, since the spectral bandwidth of the laser matches the linewidth of the vibrational mode the efficiency of the optical transition is significantly enhanced on resonance, leading to a much lower relative contribution of the non-resonant background. Second, narrowband CARS signals are typically detected on monolithic detectors such as photomultiplier tubes (PMTs) and bulk photodiodes (PDs). These detectors have bandwidths on the order of tens to hundreds of megahertz, allowing exceptionally fast imaging, while even the best broadband CARS microscope is limited to read-out speeds of about 10 kilohertz[66].

Narrowband CARS microscopy has been used to great effect particularly in biology and biomedicine[67]. Particularly good targets for CARS microscopy are lipids, which due to their long alkyl chains generate strong signals in the $\mathrm{C}-\mathrm{H}$ region of the spectrum[68-73]. Myelin morphology has been studied with CARS 

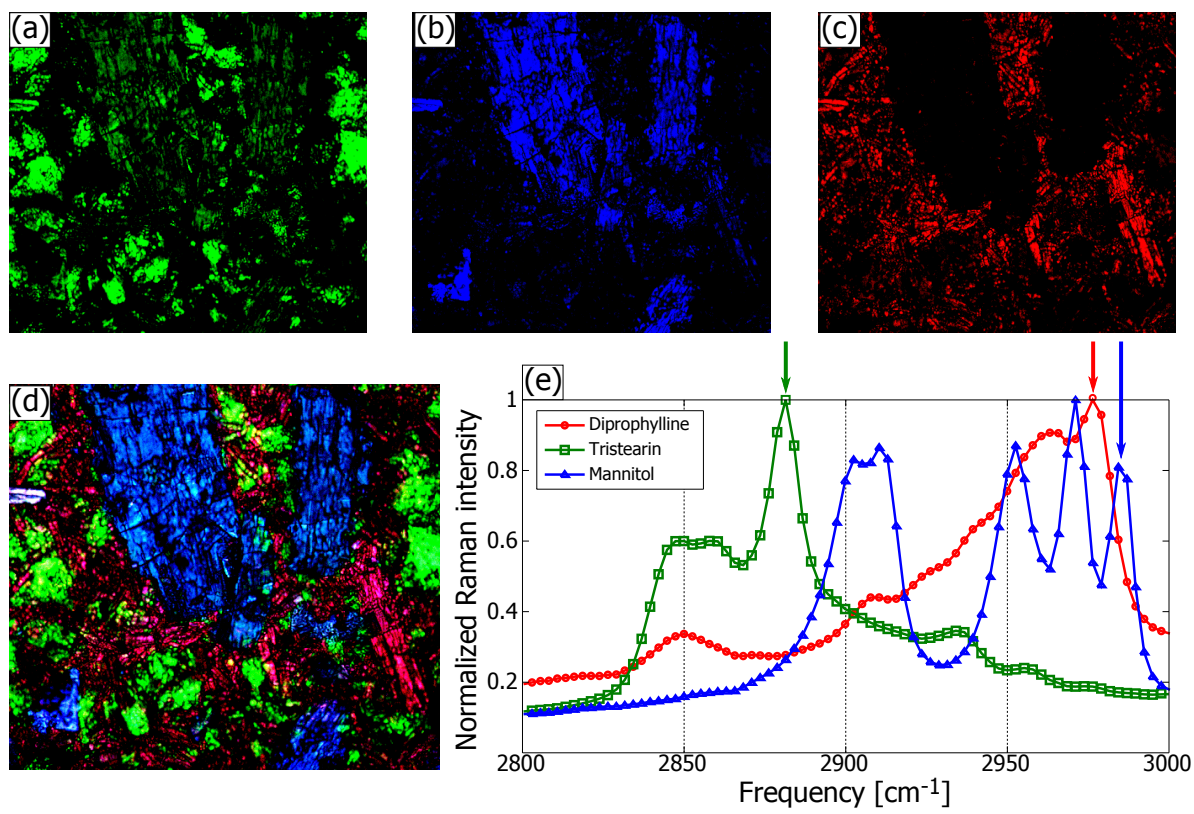

Figure 2.1. (a-c) Narrowband CARS images at the indicated frequencies in (e), roughly corresponding to tristearin, mannitol, and diprophylline, respectively. (d) Composite multi-spectral image of the three component frames. The white crystal in the upper-left of this image cannot be positively identified as one of the three constituent materials. (e) Spontaneous Raman spectra of the three materials, with arrows indicating the frequencies at which the images are recorded.

with respect to degenerative diseases and environmental toxins[74-77]. Perney et al. characterized the aggregation of polyglutamine peptides in transgenic C. elegans to elucidate their possible role in Huntington's disease[78], Garrett and her colleagues imaged the uptake of nanomedicines[79], and Fussell et al. monitored the dissolution behaviors of active pharmaceutical ingredients in real time[80].

While the good spectral selectivity of narrowband CARS microscopy is considered advantageous for many studies, the associated lack of spectral bandwidth can be detrimental. The dominant paradigm in narrowband CARS microscopy for nearly a decade was multispectral imaging, wherein specific vibrational frequencies-usually chosen based on spontaneous Raman spectra of the pure compounds of interest-were manually selected and imaged in series. While this approach works well for biological samples where most of the rel- 
evant compounds are in liquid phase, it breaks down for materials that can solidify into multiple crystalline forms. As an example of this issue from the pharmaceutical sciences, Figs. 2.1(a-c) show single-frequency CARS images of a model oral dosage form consisting of three ingredients: a triglyceride binding agent (tristearin), the active pharmaceutical agent (diprophylline), and an excipient ( $\beta$-mannitol). The spontaneous Raman spectra of these three compounds are shown in Fig. 2.1(e). The arrows point to the frequencies at which the individual images were obtained. While the specificity is generally very good, the small white crystal appearing in top left corner of the composite image (Fig. 2.1(d)) causes an analytical problem. Without further spectral information, the identification of this crystal is impossible.

An approach to resolve this problem of interpretation is to measure a vibrational spectrum for every pixel, in a process known as hyperspectral imaging. In contrast to multiplex CARS, where the spectra are recorded serially across the image[81], narrowband hyperspectral CARS acquires individual image frames at high rates with slow frequency scanning. Each individual frame of the resulting series will then contain spatially-resolved information about a specific and unique vibrational frequency. This style of vibrational imaging is called narrowband hyperspectral CARS microscopy[82, 83]. We have developed a robust system for hyperspectral CARS imaging that has already found use in a number of different fields. In this chapter we describe the classical theory of coherent Raman scattering, our experimental setup, and a number of interesting results.

\subsection{Classical theory of coherent Raman scattering}

\subsubsection{Nonlinear optics}

Optical fields impinging on a material influence the electron clouds of its constituent molecules. When this material is non-magnetic and homogeneous the propagating wave equation

$$
\nabla^{2} \tilde{E}-\frac{n^{2}}{c^{2}} \frac{\partial^{2} \tilde{E}}{\partial t^{2}}=\frac{1}{\varepsilon_{0} c^{2}} \frac{\partial^{2} \tilde{P}}{\partial t^{2}}
$$

applies, where the propagating complex electric field $\tilde{E}=A_{0} \exp [-i(k z+\omega t)]$ induces a material polarization $\tilde{P}, c$ is the speed of light in vacuum, $n$ is the refractive index of the material, and $\varepsilon_{0}$ is the free-space permittivity. Expanding 
$\tilde{P}$ into orders of $\tilde{E}$, the material polarization takes the form

$$
\tilde{P}=\epsilon_{0}\left(\chi^{(1)} \tilde{E}+\chi^{(2)} \tilde{E}^{2}+\chi^{(3)} \tilde{E}^{3}+\cdots\right) .
$$

The terms $\chi^{(n)}$ are the macroscopic material susceptibilities at various orders of the electric field which govern $(n+1)$-wave-mixing processes. The first term, $\chi^{(1)}$, is the linear susceptibility that governs the vast majority of the optical effects that we observe around us, such as linear absorption and scattering. The higher-order terms lead to nonlinear effects. The term containing $\chi^{(2)}$ is responsible for processes including second-harmonic generation, sum- and difference-frequency mixing, and DC rectification. The third-order susceptibility term $\chi^{(3)}$ is of particular interest here because it contains the lowest-order terms that describe Raman scattering.

\subsubsection{Coherent anti-Stokes Raman scattering}

In the most general four-wave-mixing scheme there are three independent fields incident on the sample

$$
\tilde{E}_{t o t}=\sum_{n=1}^{3} A_{n} e^{-i\left(k_{n} z+\omega_{n} t\right)}+\text { c.c. }
$$

where $A_{n}$ is the real amplitude of field $n, \mathbf{k}_{n}$ is its wavevector and $\omega_{n}$ is its frequency. We explicitly set $\omega_{1} \geq \omega_{2} \geq \omega_{3}$. All waves are assumed to be plane waves, with their electric fields polarized along the same axis and propagating in the $z$ direction. When we consider all time-ordering permutations of these three input fields, including all degenerate cases, where only a single output field is generated, then the third-order material polarization in Eq. 2.2 will contain 44 unique terms[84]. Dropping the tildes - we will always consider the electric fields and material polarizations as complex values unless otherwise stated - we write out $\chi^{(3)}$ from Eq. 2.2 in terms of its component fields as

$$
P^{(3)}=\epsilon_{0} \chi^{(3)}\left(\omega_{a s} ; \omega_{p},-\omega_{s}, \omega_{p r}\right) E_{p} E_{s}^{*} E_{p r} .
$$

Our notation indicates that three incoming fields at frequencies $\omega_{p}, \omega_{s}$, and $\omega_{p r}$, called the pump, Stokes, and probe fields, respectively, interact with the nonlinear susceptibility to generate a new anti-Stokes field at frequency $\omega_{a s}$ via the material polarization. The ordering of the electric fields in subsequent appearances of the material polarization will follow this pattern. The field $E_{s}^{*}$ is the complex conjugate of the field $E_{s}$. We only consider those terms of 
Eq. 2.4 that satisfy the conditions $\omega_{p}>\omega_{s}, \omega_{p r}>\omega_{s}$, and $\omega_{a s}>\omega_{p r}, \omega_{p}$. Five terms then remain:

$$
\begin{aligned}
P^{(3)} & \propto \chi^{(3)} E_{1} E_{3}^{*} E_{1} \\
& +\chi^{(3)} E_{1} E_{3}^{*} E_{2} \\
& +\chi^{(3)} E_{1} E_{2}^{*} E_{1} \\
& +\chi^{(3)} E_{2} E_{3}^{*} E_{1} \\
& +\chi^{(3)} E_{2} E_{3}^{*} E_{2} .
\end{aligned}
$$

Note that three difference frequencies are probed within with these five terms: $\omega_{1}-\omega_{3}, \omega_{1}-\omega_{2}$, and $\omega_{2}-\omega_{3}$. Practical narrowband CARS experimental systems typically use only two input fields, which eliminates four of the five terms above. The one remaining term, $\chi^{(3)} E_{1} E_{2}^{*} E_{1}$, probes only a single difference frequency, $\omega_{2}-\omega_{1}$. When this optical difference frequency coincides with that of a molecular vibration the material polarization is resonantly enhanced. To a first approximation the resonant motion of charges relative to each other in a molecule can be described as a damped, driven harmonic oscillator[85]. Molecules generally consist of more than two atoms, and as a result have numerous vibrational modes. When all of the vibrational motions are taken into account the resonant nonlinear susceptibility can be written as

$$
\chi_{R}^{(3)}=\sum_{n} \frac{A_{n}}{\Omega_{n}^{2}-\omega^{2}-2 i \omega \Gamma_{n}} .
$$

where $A$ is the amplitude of the vibrational response, $\Omega$ is the center frequency of the resonance, $\Gamma$ is the full-width-at-half-maximum (FWHM) linewidth of the resonance, and $\omega=\omega_{p}-\omega_{s}$ is the driving frequency. The sum is taken over all $n$ Raman-active vibrational modes of the molecule that generate a net change in the polarizability of the molecule. The sum frequencies $\omega_{1}+\omega_{2}$, $\omega_{1}+\omega_{3}$, and $\omega_{2}+\omega_{3}$ of the three incident fields are assumed to be far detuned from any electronic resonances, which would otherwise require the inclusion of additional terms in the resonant susceptibility[43]

In addition to this complex resonant term there is a real term that arises from the far-off-resonant, in-phase response of the molecular electron cloud to the driving field, which is added to the resonant term to form the full nonlinear susceptibility

$$
\chi^{(3)}=\chi_{R}^{(3)}+\chi_{N R}^{(3)}
$$

The resonant term is complex and is strongly dependent on driving frequency, 

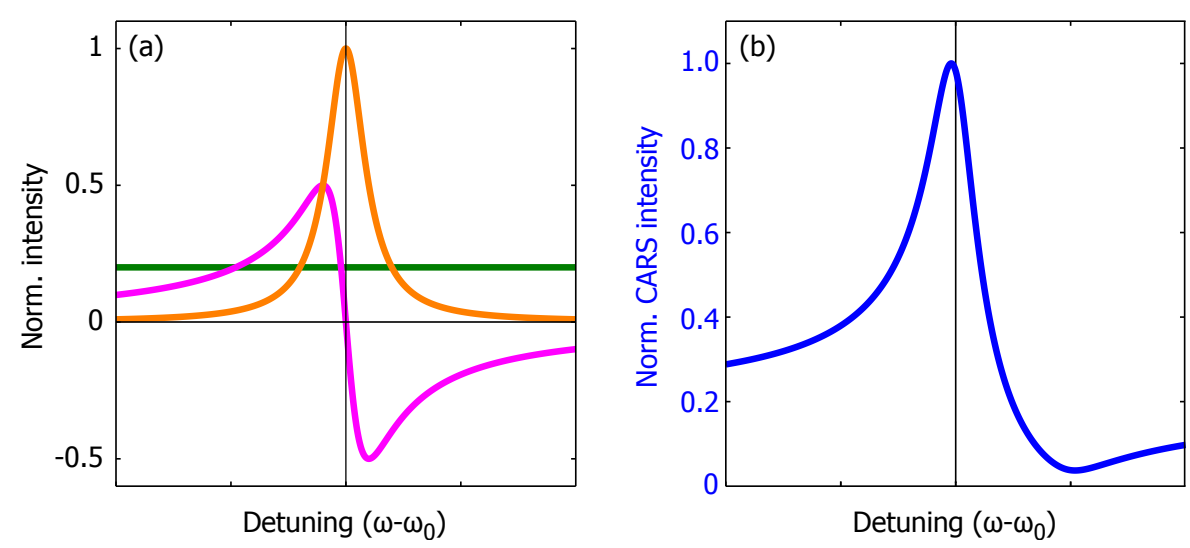

Figure 2.2. (a) The individual contributions to a single CARS resonance from the square modulus resonant component (orange), mixing term (magenta), and non-resonant background (green). (b) The full modulus-square CARS intensity spectrum. Note the plateau on the low-frequency side, the dip on the highfrequency side of the resonance, and the red-shifted peak.

while the non-resonant term is real and is largely independent of driving frequency.

Most CARS experiments are set up to measure the intensity of the antiStokes emission, which is proportional to the square modulus of the nonlinear material polarization

$$
I_{C A R S} \propto\left|\mathbf{P}^{(3)}\right|^{2}=\left|\epsilon_{0} \chi^{(3)}\right|^{2} I_{p} I_{s} I_{p r}
$$

wherein the square modulus of $\chi^{(3)}$ on the right side of Eq. 2.8 can be expanded as

$$
\left|\chi^{(3)}\right|^{2}=\left|\chi_{R}^{(3)}\right|^{2}+2 \chi_{N R}^{(3)} \Re\left[\chi_{R}^{(3)}\right]+\left|\chi_{N R}^{(3)}\right|^{2} .
$$

The CARS signal scales quadratically with the nonlinear susceptibility and linearly with the intensities of each of the three input fields. In standard experimental practice the pump and probe fields are degenerately provided by the same beam, so in many CARS experiments the signal scales quadratically with the pump/probe intensity. The first term on the right side of the equation carries fully resonant, frequency-dependent information about the sample, while the last term is purely non-resonant and, to a close approximation, frequencyindependent. The middle term results from a nonlinear mixing between the 
resonant and non-resonant components of $\chi^{(3)}$, and accounts for the asymmetric Fano lineshape of a CARS resonance compared to that of a pure Lorentzian as seen in typical spontaneous Raman scattering results. These three terms are individually plotted for a single resonance in Fig. 2.2(a), with the full squaremodulus $\left|\chi^{(3)}\right|^{2}$ shown in Fig. 2.2(b).

\subsection{Experimental details}

Good design of a narrowband CARS microscope must balance the power relationships of the anti-Stokes signal, which scales approximately inverse-quadratically with the laser pulse width, against spectral resolution, which scales proportionally with pulse length. A good compromise is found for laser pulses around $3 \mathrm{ps}$ long[43]. Both picosecond titanium-doped sapphire (Ti:sapphire) lasers[28, 86, 87] and synchronously-pumped optical parametric oscillators (OPOs)[88-90] are widely used in narrowband CARS experiments for precisely this reason. These two devices are generally pumped with the frequency-doubled output of a neodymium-doped yttrium vanadate $\left(\mathrm{Nd}: \mathrm{YVO}_{4}\right)$ laser at $532 \mathrm{~nm}$. Depending on the details of their construction, both the Ti:sapphire laser and OPO can efficiently oscillate at around $800 \mathrm{~nm}$. Conveniently, a pump wavelength of $816.8 \mathrm{~nm}$ combined with a Stokes wavelength of $1064 \mathrm{~nm}$ corresponds to a difference frequency of $2845 \mathrm{~cm}^{-1}$, which is a key marker for $\mathrm{CH}_{2}$-rich lipids. These two wavelengths, in the near-infrared, experience reduced scattering and reduced sample photodamage compared to visible light [91, 92] but remain in a spectral region where efficient microscopy optics exist.

\subsubsection{Optical setup}

For the experiments detailed in this thesis, a tandem laser system consisting of a commercial OPO (Levante Emerald, APE Berlin GmbH) synchronously pumped by the frequency-doubled output of a picosecond $\mathrm{Nd}: \mathrm{YVO}_{4}$ laser (Paladin, Coherent Inc.) was used to generate the pump and Stokes wavelengths. The OPO is a particularly interesting light source for CARS experiments for a number of reasons. Because it is synchronously pumped by a pulsed laser, its repetition rate is by definition identical to that of the pump source, precluding the external synchronization necessary for dual Ti:sapphire lasers, for example. Additionally, the nonlinear process that generates the resonating signal wavelength simultaneously produces a phase- and frequency-locked idler wavelength that can readily be extracted from the cavity. By energy-conservation 


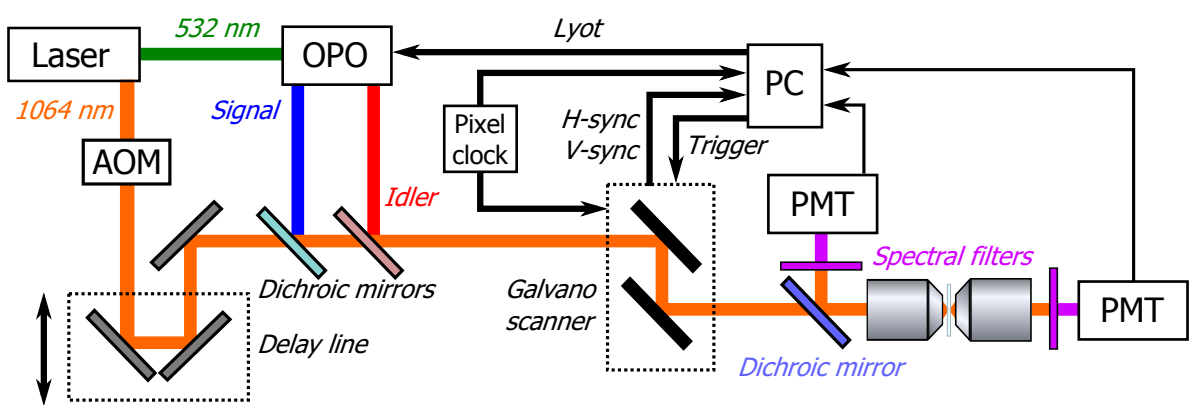

Figure 2.3. The optics of the hyperspectral CARS setup include a frequencydoubled $\mathrm{Nd}: \mathrm{YVO}_{4}$ laser pumping an optical parametric oscillator and a laserscanning inverted microscope. Three different beams are available for experiments. An acousto-optic modulator is used to switch the power of the 1064-nm beam at high frequency. Galvano scanning mirrors sweep the beams across the sample via a high-NA focussing objective. Scattered CARS signals are collected in transmission and reflection and redirected to photomultipler tubes. Synchronization signals are routed between the microscope and the OPO for hyperspectral imaging.

principles it can be shown that the output frequencies of the OPO are given by $\omega_{s}+\omega_{i}=\omega_{p}=2 \omega_{f}$, where the subscript $s$ indicates the signal, $i$ the idler, $p$ the OPO pump at $532 \mathrm{~nm}$, and $f$ the laser fundamental at $1064 \mathrm{~nm}$. The wavelength tuning window of the signal beam is coarsely selected by adjusting the temperature of the non-critically phase-matched lithium triborate (LBO) crystal. Fine selection of the signal wavelength is achieved with a rotating intra-cavity Lyot filter. The signal and idler wavelengths are both extracted from the OPO cavity and spatially separated with a dichroic mirror. These two beams can then be re-combined with each other or with the laser fundamental beam in any permutation. In signal-fundamental mode at high difference frequencies $\left(\approx 3000 \mathrm{~cm}^{-1}\right)$ the tuning range of the signal wavelength is about $180 \mathrm{~cm}^{-1}(15 \mathrm{~nm})$, with a spectral resolution of less than $1 \mathrm{~cm}^{-1}$ and Lyot step resolution of $0.7 \mathrm{~cm}^{-1}$. The Lyot filter step size can be adjusted in integer increments of the minimum step, up to over $100 \mathrm{~cm}^{-1}$. In this chapter, all experiments were performed with the signal-fundamental combination unless otherwise noted.

The three beams are individually conditioned to have appropriate parameters for the laser-scanning microscope (modified IX71 frame with FV300 scan unit, Olympus Inc.). Telescopes are used to adjust the beam diameter and divergence, waveplates control the polarizations, and attenuators in each beam 
are used to change the powers. Delay stages in the $1064 \mathrm{~nm}$ and idler beams are used to ensure that temporal overlap between the pulses in each beam is achieved, and the three beams are overlapped on a series of two dichroic mirrors. The combined beams are then launched into the laser-scanning microscope. Following a galvanometric scan mirror pair, the beams pass through a scan lens set and tube lens before travelling through a long-pass dichroic mirror and finally impinging on the back aperture of an infinity-conjugated objective lens and focusing into the sample. Various mirrors and objectives can be installed to fulfill the requirements of different applications. Light that is transmitted through the sample is collected by a different objective and relayed to an array of detectors, including PMTs (R3896 and R943-02, both Hamamatsu Inc.) and PDs (FDS1010, FDS100, and FGA21, all ThorLabs Inc.), via dichroic mirrors and spectral filters. Back-scattered light is initially spectrally filtered by the long-pass dichroic; any reflected light is then directed through additional spectral filters before landing on a PMT (R3896, Hamamatsu Inc.). A schematic diagram of the optics used for hyperspectral CARS is shown in Fig. 2.3. Additional components mentioned previously but not depicted in Fig. 2.3 will appear in later chapters. The anode of the R3896 $\mathrm{PMT}$ in the transmission direction feeds directly into a 1-M $\Omega$ transimpedance amplifier, while the epi-located R3896 PMT anode is connected to a $10-\mathrm{M} \Omega$ transimpedance amplifier.

\subsubsection{Acquisition and synchronization}

The second key component of the hyperspectral CARS microscopy system is the acquisition software and synchronization electronics. For hyperspectral imaging we select and fix the OPO crystal temperature and adjust only the Lyot filter. By operating in this mode we trade tuning bandwidth for acquisition speed. The motion of the Lyot filter is synchronized to the microscope scan unit via transistor-transistor logic (TTL) signals. The FV300 scan unit is linked to a FV5-PSU power supply unit which has digital hardware inputs and outputs with various functionalities. Among the outputs are TTL lines for the line and frame scan directions. The line signal switches to TTL HI $(>2.6 \mathrm{~V})$ when a line along the $x$-axis of the image is actively being scanned, and goes TTL LO $(<0.4 \mathrm{~V})$ when the $x$-axis galvano mirror is on the return stroke to its origin. Likewise, the frame signal switches to TTL HI while the image is being acquired, and switches to TTL LO when the $y$-axis galvano mirror is returning to its origin in preparation for the next frame. During the acquisition of a frame 


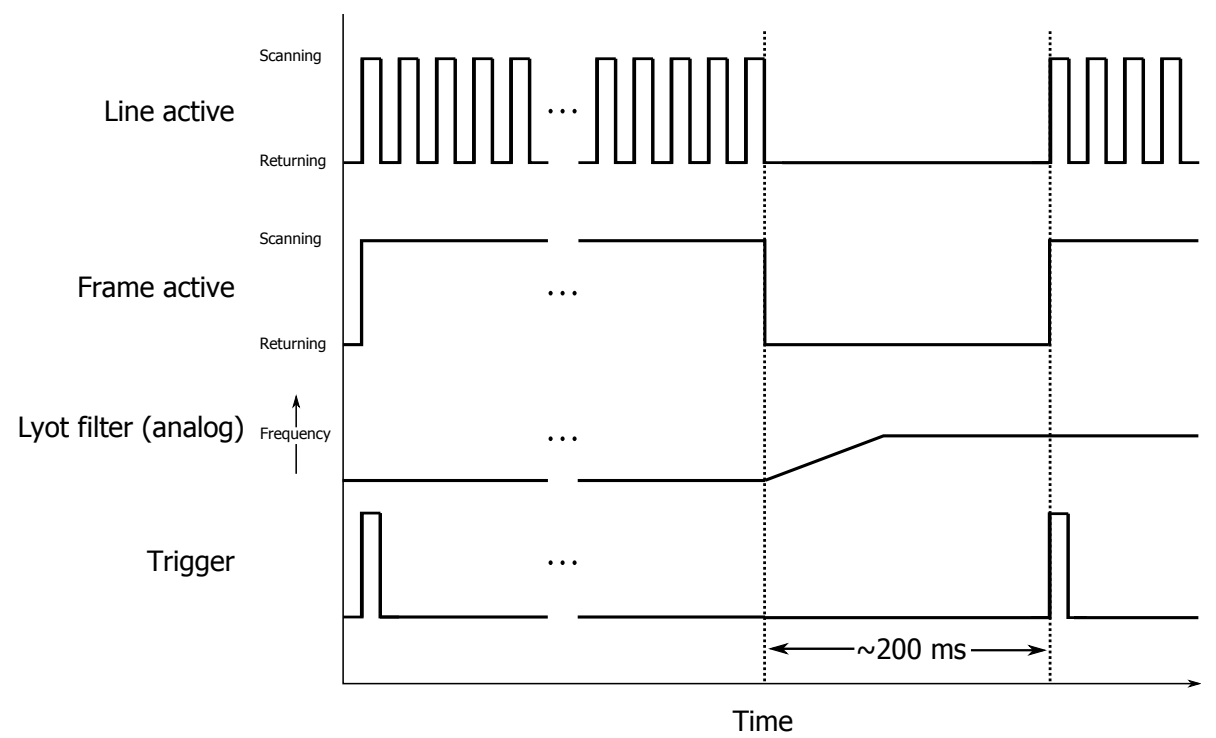

Figure 2.4. Traces of the signals involved in one frame of the hyperspectral scanning routine. A digital trigger signal to the microscope initiates image acquisition, consisting of $\mathrm{N}$ lines per frame, with each line containing $\mathrm{N}$ pixels (pixel clock not shown). At the end of the frame a signal is sent to the Lyot filter to shift to the next frequency, an operation which takes about $150 \mathrm{~ms}$. After allowing time for the Lyot filter to move and settle, a new trigger signal is sent to begin the next frame acquisition. Data is not recorded during the line return strokes.

of the hyperspectral image the Lyot filter is held in constant position until the frame signal goes TTL LO, at which point the FluoView software begins idling until it registers a TTL HI pulse on the FV5-PSU Trig 0 TTL line. A second computer running custom LabVIEW software registers the TTL LO frame signal and immediately sends a rotation command to the Lyot filter control unit via RS232, for which the maximum latency is about $150 \mathrm{~ms}$. To ensure that the next frame does not begin until the Lyot filter motion has completed and settled the LabVIEW computer idles for $200 \mathrm{~ms}$ before a TTL HI signal is given to the FV5-PSU Trig 0 TTL port. The entire process then repeats until a pre-set number of frames has been acquired. The vibrational frequency and signal power level are recorded for each frame of the hyperspectral image to calibrate and correct the data in post-processing. 


\subsubsection{Hyperspectral projection}

A final component to the hyperspectral CARS system is in the data analysis. Because CARS is by definition a nonlinear process that involves mixing of resonant and non-resonant components it is a serious challenge to extract any quantitative information from experimental data. The use of phase-retrieval algorithms is a viable strategy, but the two main approaches - the Maximum Entropy Method (MEM)[93, 94] and Modified Kramers-Kronig (MKK)[95]both implicitly require large spectral bandwidths so that they can estimate the background error phase. In our setup, the tuning bandwidth is rarely wide enough to enable this calculation. As a result, we have avoided quantitative interpretation of the hyperspectral CARS data and instead developed a qualitative analysis method that relies on visual contrast. The data acquired with the system described above can be represented as a three-dimensional array of data points $(x, y, \omega)$, with two spatial dimensions forming the horizontal and vertical axes of the image and the third dimension representing vibrational frequency. This array is referred to as a hyperspectral datacube. The goal is to create a single two-dimensional image where the individual components appear with high contrast. We achieve this result via a three-step process. First, the pixel intensity values are normalized to the global maximum, so that a fully dark pixel will have a grey value of zero, and the brightest pixel(s) in the image will have a grey value of unity. Second, every vibrational frequency is assigned its own unique color from a color look-up table (LUT). Each pixel in the datacube will then have a hue determined by its frequency and a saturation given by its (normalized) intensity. Finally, the entire stack is additively mixed along the frequency axis with a maximum intensity projection. The resulting two-dimensional image contains pixels whose colors are the qualitative representation of the vibrational spectrum at each spatial location within the sample. Sharp peaks result in brilliant, high-contrast colors, while broad spectral features result in low-saturation, pale colors. This projection method is shown in Fig. 2.5, and has been implemented as a set of custom scripts in Image J that call upon the McMasters Biophotonics Facility, Ontario (MBF) plug-in library.

The choice of the color map assigned to the hyperspectral datacube is arbitrary and does not affect the underlying data. For well-characterized samples it is possible to tailor an optimal LUT that maximizes contrast, but in many cases the molecular composition is not known a priori. It is therefore most useful to choose a LUT that maximizes the contrast of individual spectral fea- 


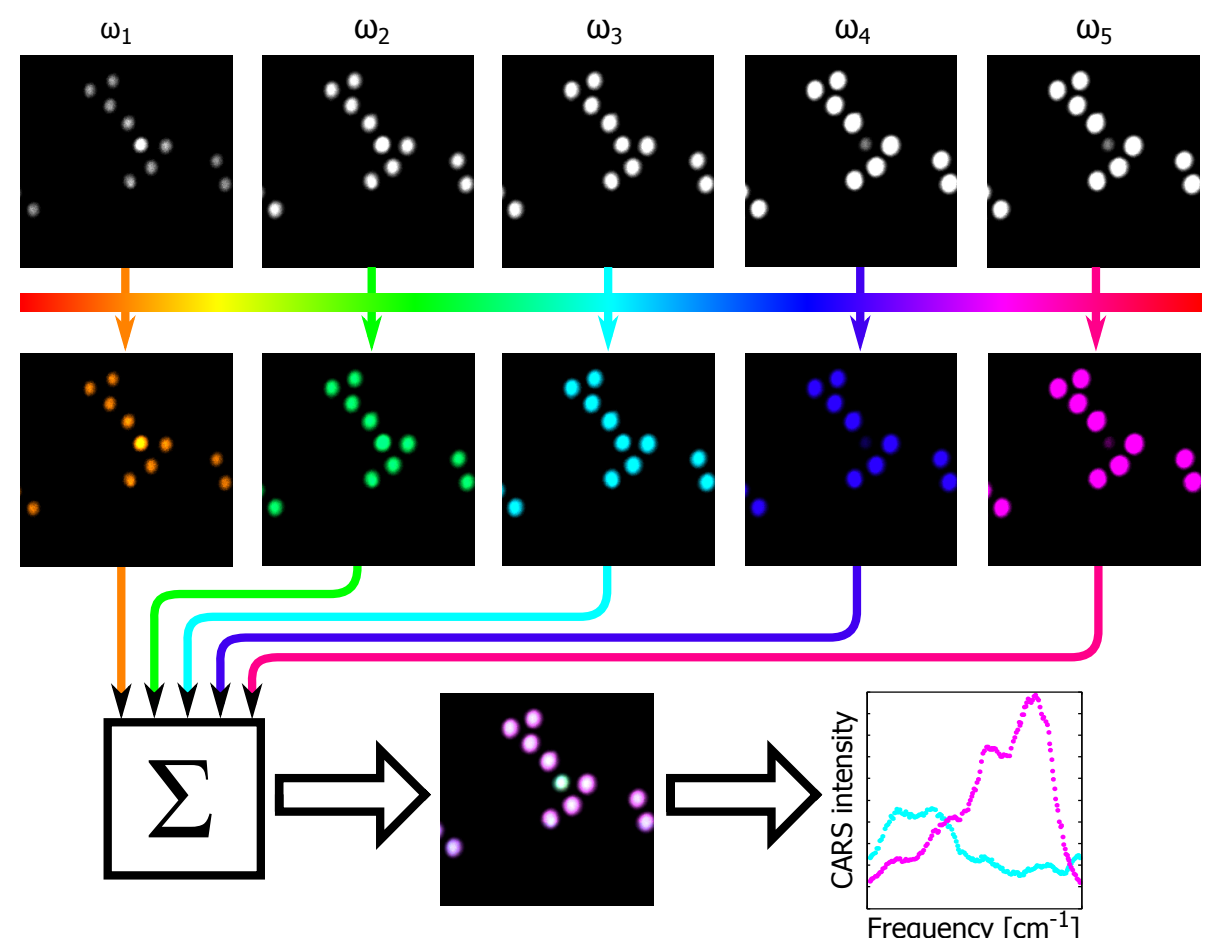

Figure 2.5. The hyperspectral projection method. First, a stack of images at a sequence of vibrational frequencies is recorded (top). Second, the vibrational spectra are translated into the visible spectrum by applying a unique color lookup table (center). Third, the spectrum of each spatial pixel is summed to yield the final projected image (bottom-left). The CARS spectrum from any given region can be extracted from the original hyperspectral data set (bottom right).

tures regardless of the sample. The most general LUT contains the full visible spectrum with the primary colors equally spaced in frequency. The immediate problem with this LUT is directly related to how our eyes perceive colors. Human trichromic receptors pick out red, green, and blue (RGB) as the primary colors. As a result of this trichromicity, an object that emits light with equal amounts of pure blue $(\approx 420 \mathrm{~nm})$ and green $(\approx 530 \mathrm{~nm})$ will appear nearly identical to an object that radiates cyan light $(\approx 480 \mathrm{~nm})$ with equal integrated intensity. This chromic ambiguity is demonstrated in Fig. 2.6(LUT 1) for two different amino acids, one of which has peaks in the blue and green sections of the LUT, and the other of which has a single dominant peak in the cyan. To resolve this ambiguity we introduce a pair of new LUTs that compress the 
distance between primary colors along the frequency axis of the hyperspectral datacube. As a result of the compression the spectral peaks of the various compounds lie at different combinations of colors in the LUT, and as a result the projections show up with significantly different colors. The next effect of using multiple LUTs is shown in Figs. 2.6(LUT 2) and (LUT 3). With using these three LUTs the likelihood of two compounds sharing the same colors in all three projections becomes small, except in cases where the individual spectra are highly similar. However, small variations in the properties of the driving laser fields can result in significant changes in the projected images, so when multiple hyperspectral images are to be directly compared to each other care must be taken to ensure that all experimental parameters are kept identical from image to image.

\subsection{Results}

\subsubsection{Rapid identification of heterogeneous mixture components}

The primary application of this hyperspectral projection method is the rapid visual analysis of the distribution of vibrational spectra within a sample covering areas up to hundreds of microns across. To demonstrate this strength we imaged a set of samples containing crystalline amino acids. Pure ( $>95 \%)$ amino acids were obtained from Sigma Aldrich. Control hyperspectral CARS images of the pure amino acids were acquired, and seven were chosen for this demonstration for their lack of multiple stable crystal forms and for their relatively similar CARS emission intensities. These seven amino acids are glutamine (Gln), histidine (His), isoleucine (Ile), Methionine (Met), phenylalanine (Phe), threonine (Thr), and valine (Val). A heterogeneous mixture was produced by mixing a few milligrams of each of these seven amino acids in a beaker and gently grinding the mixture until the average particle size was observed to be less than $100 \mu \mathrm{m}$ under a white-light inspection microscope. All samples were sealed under ambient atmosphere between two clean cover glasses to prevent extensive oxidation or hydration.

For these experiments only back-scattered CARS signals are recorded. Amino acid crystals are generally colorless but are highly scattering, which is not generally conducive to transmission experiments. A 40×, 0.9-NA infinity-conjugated air objective (Apochromat /340, Olympus Inc.) focuses the pump and Stokes beams into the sample and collects back-scattered CARS signal. Any light 

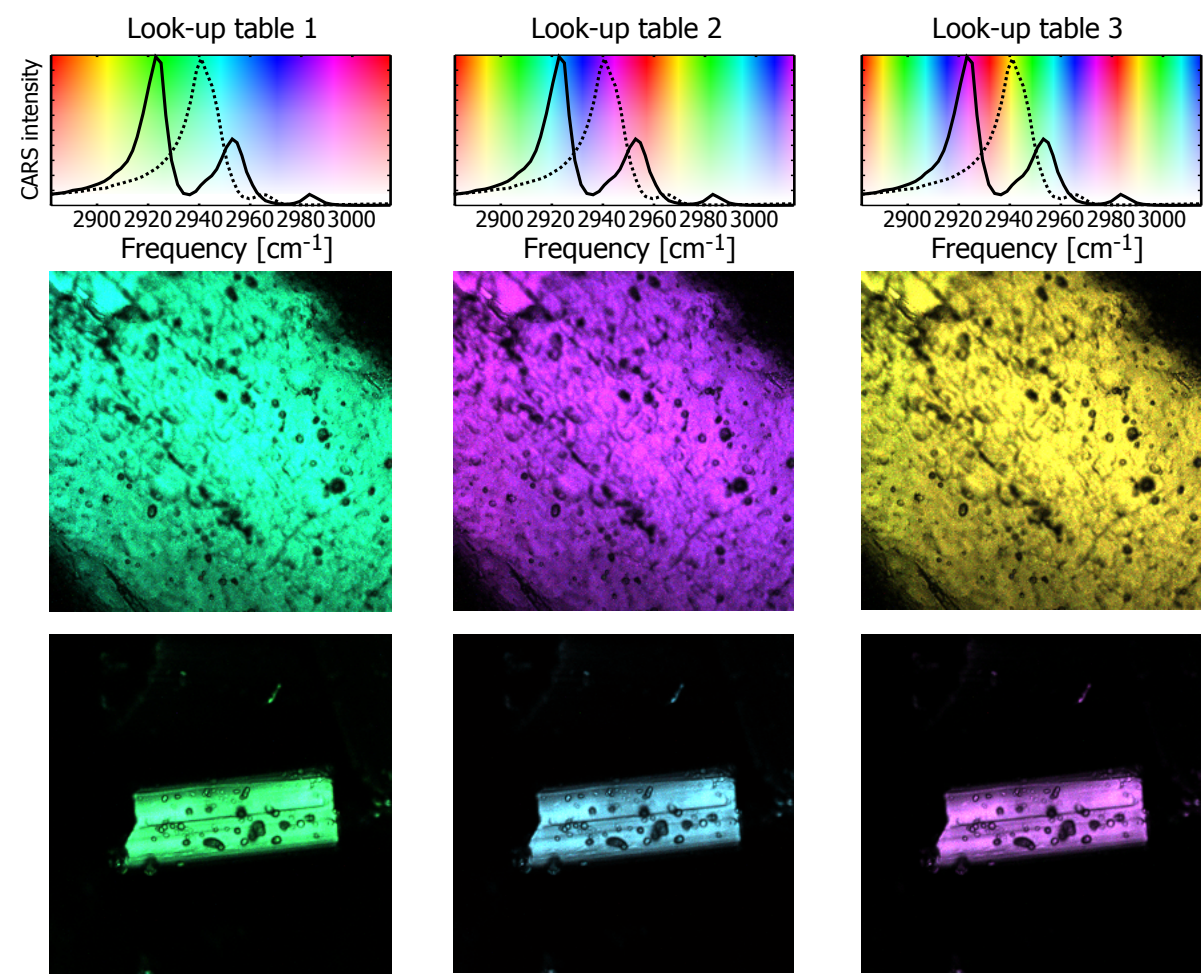

Frequency $\left[\mathrm{cm}^{-1}\right]$

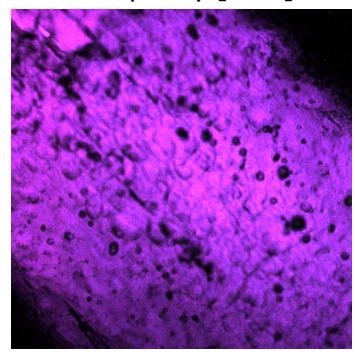

Frequency $\left[\mathrm{cm}^{-1}\right]$
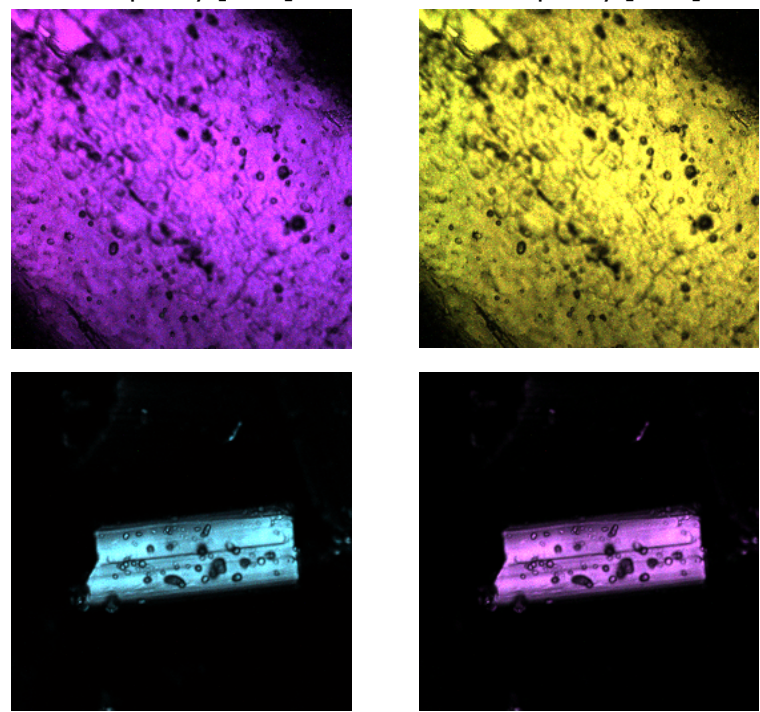

Figure 2.6. For a given LUT two different spectra can appear with the same color in the hyperspectral projection. Using multiple non-equivalent LUTs overcomes this chromic ambiguity, as shown here for histidine and glutamine crystals. The solid spectra correspond to the glutamine crystals in the bottom row of images; the dotted spectra are histidine.

with wavelength shorter than $770 \mathrm{~nm}$ is reflected from a dichroic mirror and is then additionally filtered by two short-pass filters (ET750SP, Semrock) and two band-pass filters (HQ660/40, Chroma) before hitting the epi-PMT. Power levels of the pump and Stokes beams are kept at reasonable levels so as to not photodamage the samples. Maximum pump power is $50 \mathrm{~mW}$ on the sample and decreases as the OPO is tuned away from its gain maximum, while the average Stokes power is maintained at $30 \mathrm{~mW}$.

Calibration hyperspectral images of the pure amino acids containing $256 \times 256$ pixels and recorded over 67 frequencies (covering the spectral range $2880 \mathrm{~cm}^{-1}$ to $3020 \mathrm{~cm}^{-1}$ ) were produced in 1.6 seconds per frame. The resulting hyperspectral projections are shown in Figs. 2.7 for all three of the LUTs described 

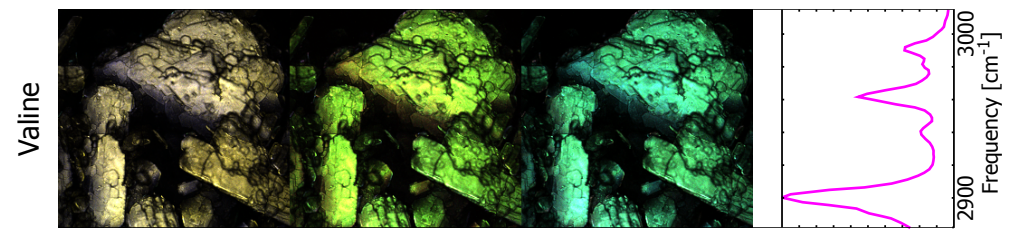

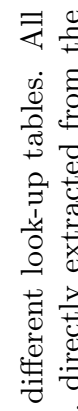
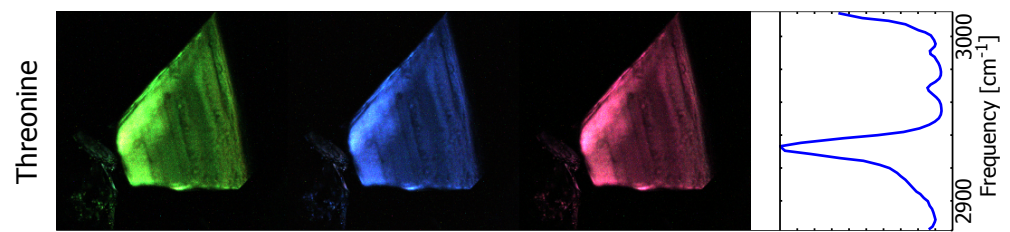

\&
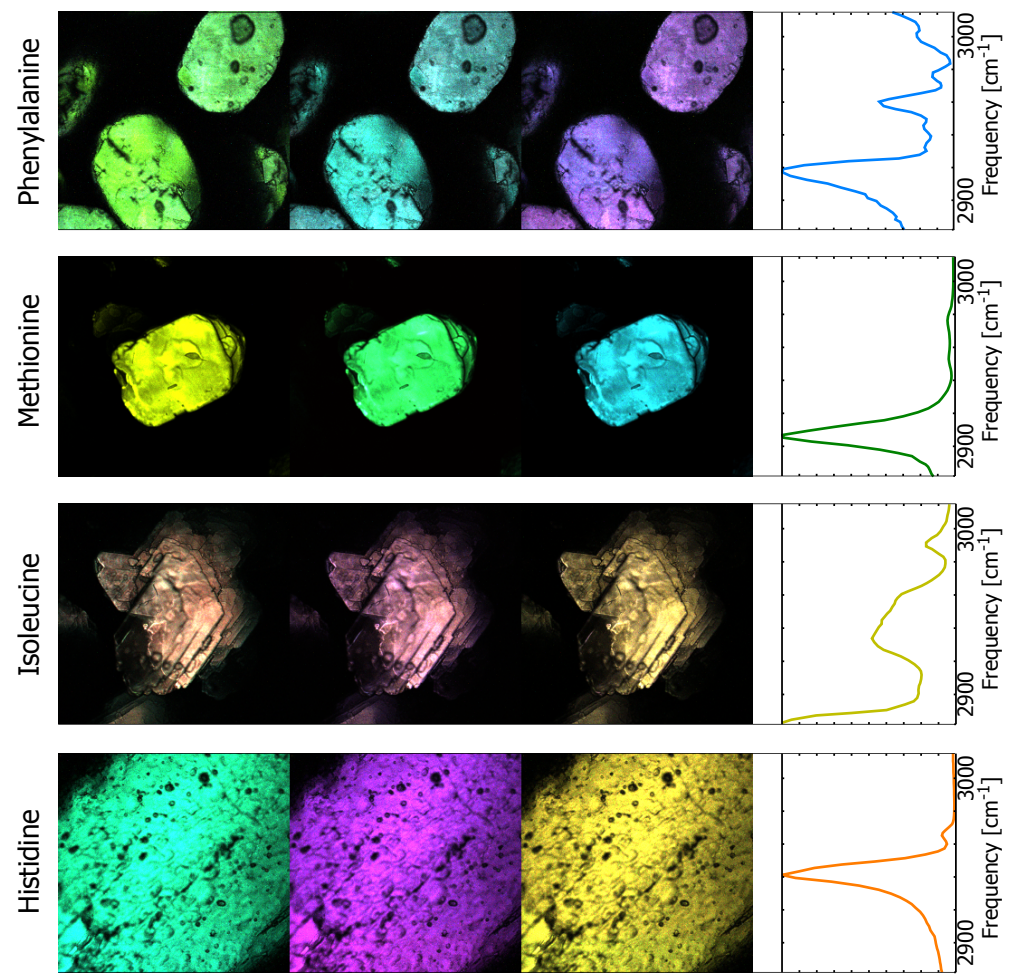

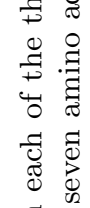

壴索

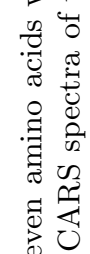

句

प.

:

苍远

를

苟莕

क्ते घं.

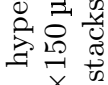

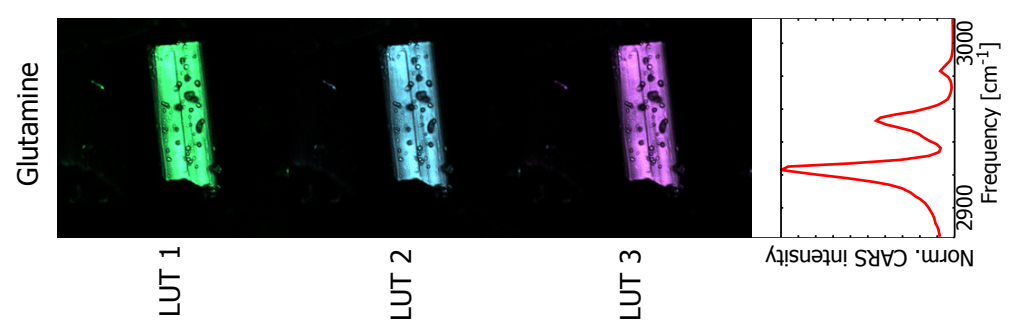

ํํ류 월

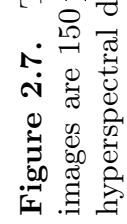



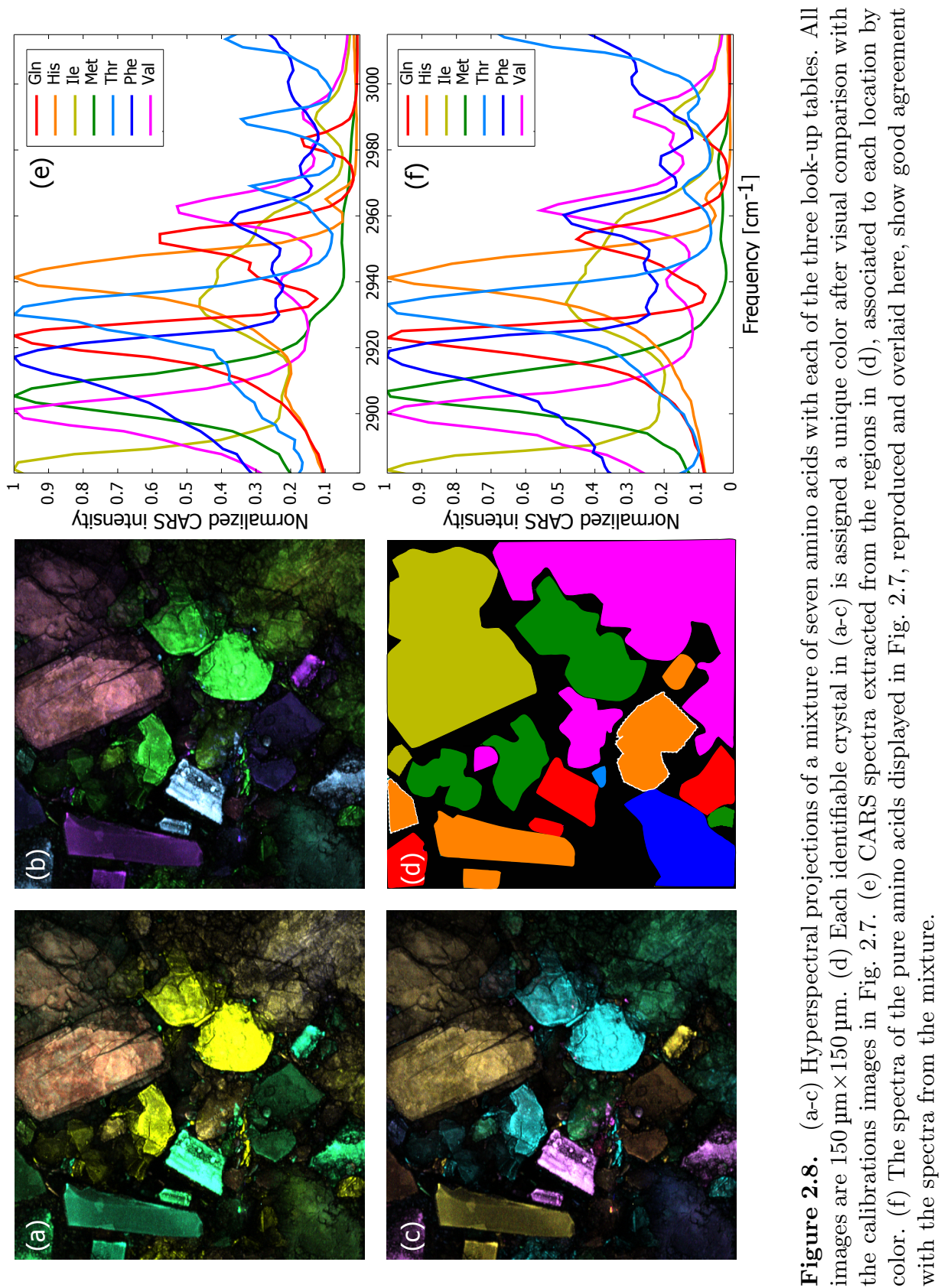

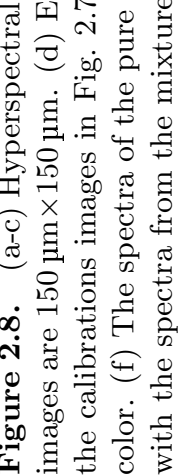


in the previous section; the colors in the hyperspectral projection of the mixed sample can then be directly and visually compared with the calibration data for the rapid identification of the different compounds. The CARS spectra of each of the seven pure amino acids are shown directly beneath their respective projection images. All spectra are directly extracted from the hyperspectral datacubes, they have been corrected for changes in the power of the OPO signal beam as a function of wavelength and locally normalized in intensity to provide a qualitative comparison of peak locations and relative intensities. It is striking that although there can be two compounds with a very similar major peak - for example, histidine at $2932 \mathrm{~cm}^{-1}$ and threonine at $2940 \mathrm{~cm}^{-1}$ - the projection images provide sufficient contrast to distinguish between the two due to the extra threonine feature at $3020 \mathrm{~cm}^{-1}$. Similarly, methionine and valine both have prominent features around $2900 \mathrm{~cm}^{-1}$, but can be readily separated by the manifold of valine features between $2920 \mathrm{~cm}^{-1}$ and $3000 \mathrm{~cm}^{-1}$ that does not exist in methionine.

The power of this analysis method is best demonstrated in a hyperspectral CARS image of a mixture all seven amino acids. This image contains $512 \times 512$ pixels, with each frame recorded in 4.4 seconds (1.1 seconds per subframe with four averages), over the same frequency range as the calibration images. The resulting projections are shown in Fig. 2.8(a)-(c). The readily identifiable crystals are represented monochromatically in Fig 2.8(d) with the same colors as their spectra in Figs. 2.8(e) and (f). The calibration spectra from Figs. 2.7 are reproduced in Fig. 2.8(e), while those extracted from the mixed hyperspectral datacube are shown in Fig. 2.8(f). The agreement between the reference spectra and the mixed spectra is generally very good, with few exceptions. These slight differences between the pure reference spectra and the mixed spectra might be due to any number of effects, including local changes in the crystal structures of the different objects due to the grinding action, or orientation of the crystals into planes that were not present in the reference images.

\subsubsection{In situ analysis of crystal polymorphs}

When samples contain multiple compounds, the chance of one of those compounds existing in more than one crystal form is not insignificant. Crystal polymorphisms and molecular isomerisms are widespread in nature, both of which can lead to extensive changes in the vibrational spectrum of the material. Molecules that contain significant hydroxyl, primary and secondary amine, and amide groups are particularly prone to polymorphisms, since they can easily 
form hydrogen bonds in a variety of configurations with other nearby molecules. Additionally, non-centrosymmetric crystals exhibit native birefringence, which is related to various Cartesian terms of the nonlinear $\chi^{(3)}$ susceptibility tensor containing different natural resonance frequencies[36]. As a result of this variation of $\chi^{(3)}$ with respect to the driving field polarization, crystals can exhibit different spectra simply as a function of their alignment with the driving laser fields even when the pump and Stokes fields are linearly co-polarized. Both of these two common material properties can significantly influence the vibrational response of a sample, and render traditional multispectral CARS microscopy methodologies obsolete. Pharmaceutical scientists in particular are keenly interested in locating polymorphs within a sample over large areas, with sub-micron resolution, and at high speed.

For a model system we use an oral dosage form as described in Chapter 1 containing a binding matrix of the triglyceride tristearin (with three fully saturated $\mathrm{C} 18$ : 0 side chains), the bronchodilatory active pharmaceutical ingredient (API) diprophylline, and the $\beta$ polymorph of the sugar alcohol mannitol as an excipient. All of these compounds are first analyzed individually using hyperspectral CARS microscopy to determine their individual nonlinear vibrational characteristics.

\section{Diprophylline}

Widely used for the treatment of asthma, diprophylline is a chiral xanthine derivative that is known to display polymorphism[96]. However, only two are reported to be stable at room temperature, and they are unique to racemic and enantiopure samples. We use a racemic mixture, so we only expect one stable polymorph, with a small residual amount of the metastable form. A hyperspectral CARS image of a random distribution of diprophylline crystals reveals that there are no fewer than three independent spectra present. Shown in Figs. 2.9(a)-(d) are two of the three hyperspectral projection images. Frames (a) and (b) were obtained with the pump and Stokes fields both linearly polarized vertically, and frames (c) and (d) were acquired with the pump fields polarized horizontally. Spectra of four different crystals are plotted at right in Figs. 2.9(e) and (f), with the color and style of the spectral curves corresponding to those of the arrows in the images.

To understand this spectral behavior we examine the structure of the nonlinear susceptibility in more detail. The susceptibility that governs a nonlinear process can include fields that are polarized in different directions. In partic- 

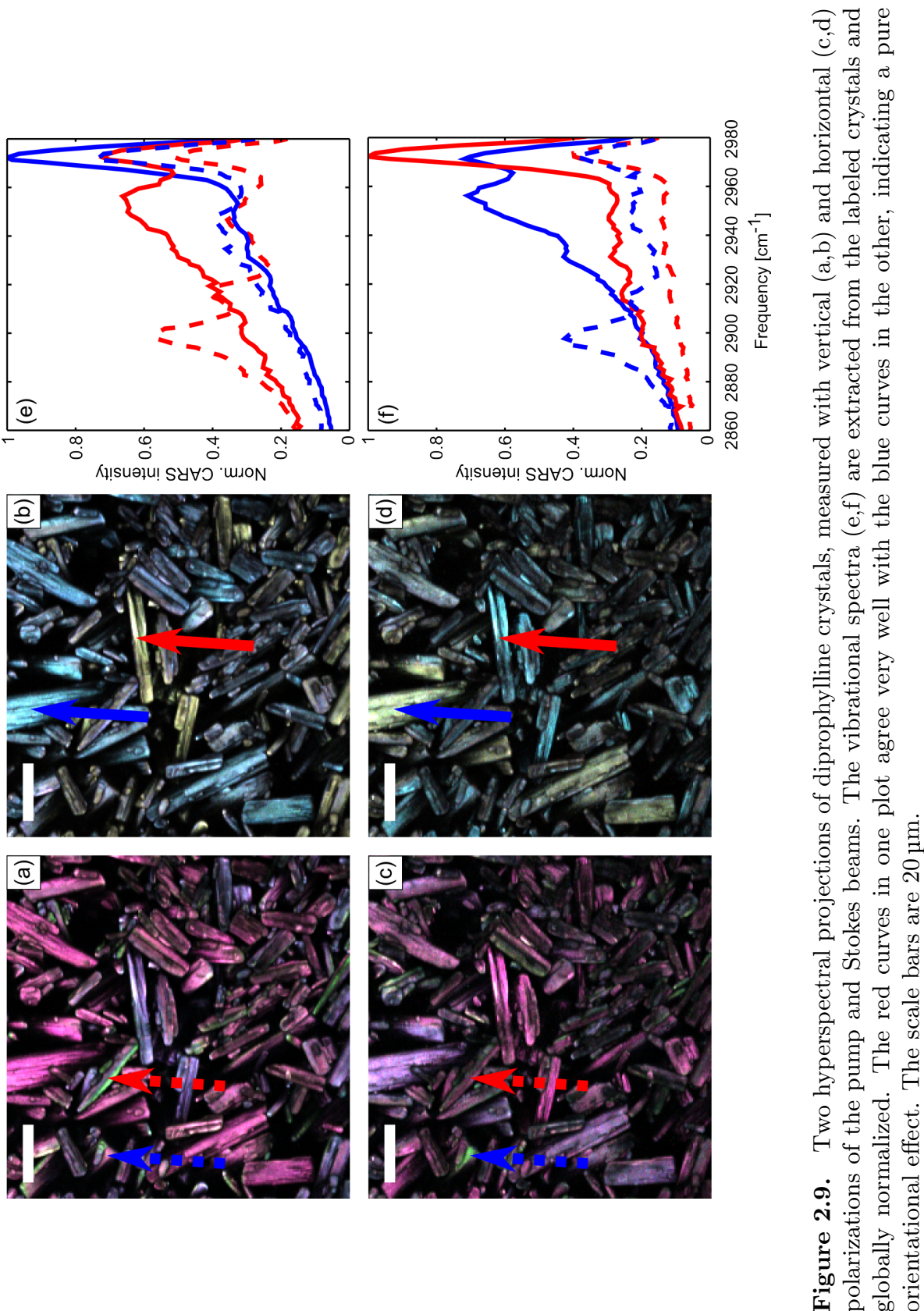

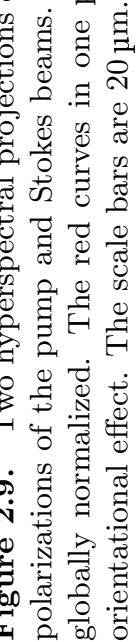


ular, the third-order susceptibility responsible for CARS is notated as $\chi_{i j k l}^{(3)}$, where the subscripts indicate the polarization of each field in the interaction in the order given in Eq. 2.4. Each of these subscripts can take the value of $x$ or $y$ for an electric field propagating in the $z$ direction. Under this condition, there are only three independent susceptibilities

$$
\chi_{x x x x}^{(3)}=\chi_{y y y y}^{(3)}=\chi_{x x y y}^{(3)}+\chi_{x y x y}^{(3)}+\chi_{x y y x}^{(3)} .
$$

Solid crystalline materials are rarely isotropic. The stable racemic diprophylline polymorph is part of the monoclinic crystal system, with a specific space group of P2[96]. This space group has a total of 41 independent susceptibility elements[84]. It is therefore no surprise that multiple CARS spectra are recorded for a pure sample. To simplify the interpretation of the data we assume that the polarizations of the individual fields are identical and precisely oriented along the crystal axes. Two unit cell axes are orthogonal in a monoclinic system[97], and we define these two axes to be $X$ and $Y$. The third unit cell axis is not orthogonal to either of these two dimensions; however, we define it to be $Z$ for brevity. The hyperspectral data shown in Figs. 2.9 are either taken with field polarizations $i=k=j=l=x$ or $i=k=j=l=y$. We do not analyze the polarization of the anti-Stokes field (the $i$ subscript), but the susceptibility components $\chi_{x y y y}^{(3)}$ and $\chi_{y x x x}^{(3)}$ are zero $[98,99]$. To describe the response of the material, taking into account both the crystal orientation and the field polarization, we use the notation $\chi_{N, i j k l}^{(3)}$, where $N=X, Y, Z$ is the crystal coordinate. By symmetry arguments $\chi_{X, x x x x}^{(3)}=\chi_{Y, y y y y}^{(3)}$ and $\chi_{Y, x x x x}^{(3)}=\chi_{X, y y y y}^{(3)}$.

From the plots in Fig. 2.9(e) we assign the solid blue spectrum (cyan crystals in (b)) to the $\chi_{Y, y y y y}^{(3)}$ component, while the solid red spectrum (yellow crystals in (b)) represent the $\chi_{X, y y y y}^{(3)}$ component. The magnitude of the $z$ field polarization component is assumed to be negligible compared to the $x$ and $y$ components[37]. A nearly exact one-to-one switch of the crystal colors between Figs. 2.9(b) and (d) indicates that the spectral variations which result in the yellow and blue hues are entirely polarization dependent. Cyan crystals in Fig. 2.9(b) appear yellow in Fig. 2.9(d), and the spectrum extracted from these crystals matches closely with the $\chi_{X, y y y y}^{(3)}$. All of the frames in Figs. 2.9 were recorded in epi-detection, and the long-pass dichroic mirror that reflected the back-scattered anti-Stokes field has a relatively strong chromatic effect on the transmitted pump fields. Differences in the relative intensities of the various crystals can be partially attributed to this optic, with a small additional con- 
tribution from residual misalignment of both the crystal orientations and laser polarizations from the true laboratory vertical and horizontal axes.

In addition to the two spectra described above, which could be readily extracted from Figs. 2.9(b) and (d), there is an additional spectrum that can be found in minority quantities in Figs. 2.9(a) and (c) as a green/magenta pair. The dashed red and blue arrows point to two regions where this color pair exists; the associated spectra for these crystals are represented by dashed blue and red curves in Figs. 2.9(e) and (f). It is apparent that the green color results from a spectrum that is highly distinct from those of the $\chi_{X, y y y y}^{(3)}$ and $\chi_{Y, y y y y}^{(3)}$ susceptibilities. The most striking new feature is the peak at $2895 \mathrm{~cm}^{-1}$, with a smaller additional feature at $2912 \mathrm{~cm}^{-1}$. We assign this new spectrum to the $\chi_{Z, x x x x}^{(3)}$ component. The $\chi_{Z, y y y y}^{(3)}$ component appears to yield a spectrum that is a combination of $\chi_{Y, y y y y}^{(3)}$ and $\chi_{X, x x x x}^{(3)}$. Scattered throughout the sample are a number of other crystals whose orientations are at non-orthogonal angles to the laboratory axes. The spectra of these crystals are nonlinear mixtures of the various orthogonal spectra, and so cannot be readily analyzed.

\section{Tristearin}

Tristearin is a waxy material with a melting point of about $65^{\circ} \mathrm{C}$. It is known to have at least six polymorphs, of which three - the $\alpha, \beta$, and $\beta$ ' forms - are stable at room temperature[100]. Of these three stable polymorphs, the $\alpha$ form has the lowest melting temperature and the lowest enthalpy of fusion, and appears as the first solid component in a cooling melt. We melted a sample of tristearin at $80^{\circ} \mathrm{C}$ and cooled it slowly to room temperature to create a sample composed primarily of the $\alpha$ polymorph. A comparison of the raw and recrystallized tristearin samples is shown in Fig. 2.10. Two of the hyperspectral CARS projections are shown in (a) and (b), with the spectra of the indicated locations from the two images in $(\mathrm{c})$ and $(\mathrm{d})$. A pair of spectra are readily apparent in the raw tristearin, with the principal difference being the ratio of the $2845 \mathrm{~cm}^{-1}$ and $2880 \mathrm{~cm}^{-1}$ peaks. On the other hand, the hyperspectral projection of the recrystallized tristearin shows only minor color variations between different regions of the sample, correlating to slight differences in the $2845 \mathrm{~cm}^{-1} / 2880 \mathrm{~cm}^{-1}$ ratio. In all cases, the peak at $2880 \mathrm{~cm}^{-1}$ is far weaker than the one at $2845 \mathrm{~cm}^{-1}$, leading us to conclude that a $2845 \mathrm{~cm}^{-1}: 2880 \mathrm{~cm}^{-1}$ ratio higher than one indicates the $\alpha$ form, while a ratio less than one represents the $\beta$ or $\beta$ ' form. Further, the $\beta$ form is more thermodynamically stable and likely has a higher degree of microscopic order, indicating that for lipids 

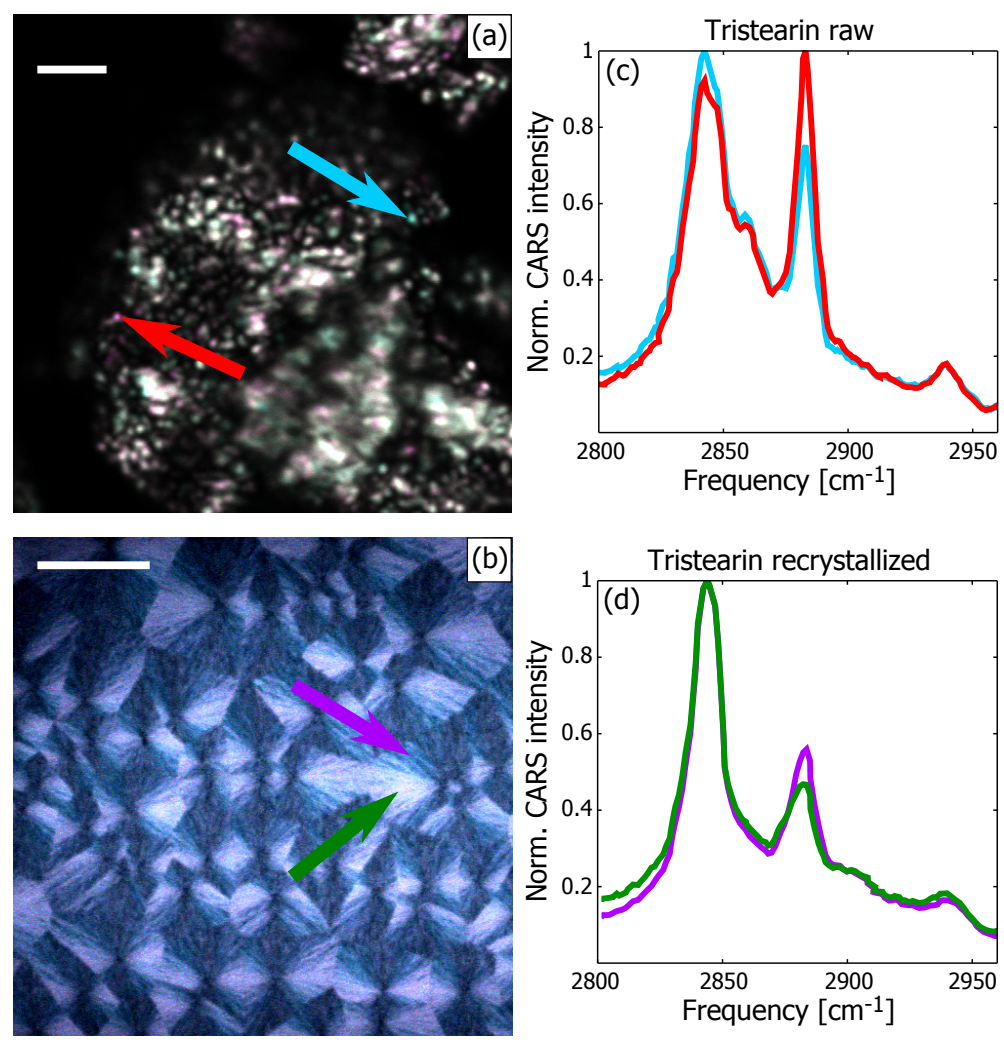

Figure 2.10. Projections of tristearin hyperspectral CARS images before (a) and after (b) melting at $80^{\circ} \mathrm{C}$ followed by cooling to room temperature. Spectra are extracted from the indicated regions and individually normalized to show the significant differences in the ratio of the $2845 \mathrm{~cm}^{-1}$ and $2880 \mathrm{~cm}^{-1}$ peaks. The scale bar in the top image is $10 \mu \mathrm{m}$, and in the bottom image is $80 \mu \mathrm{m}$.

and lipid-like compounds the $2880 \mathrm{~cm}^{-1}$ peak may be a key marker of bulk crystallinity.

\section{Mannitol}

Mannitol is a six-carbon sugar alcohol that is widely used in the food and pharmaceutical industries. Each carbon atom in the chain contains a hydroxyl functional group, allowing a variety of hydrogen-bonding networks to form. The flexibility of this small molecule further enables it to re-orient into a variety of configurations. Both of these characteristics result in the formation of different 

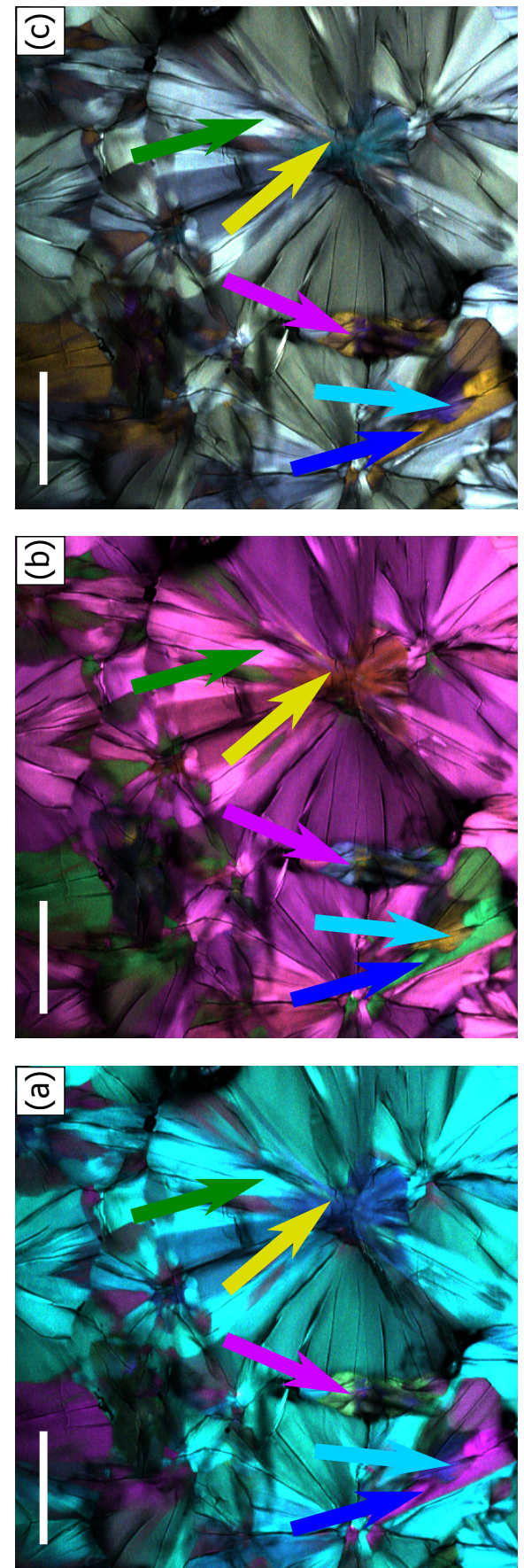
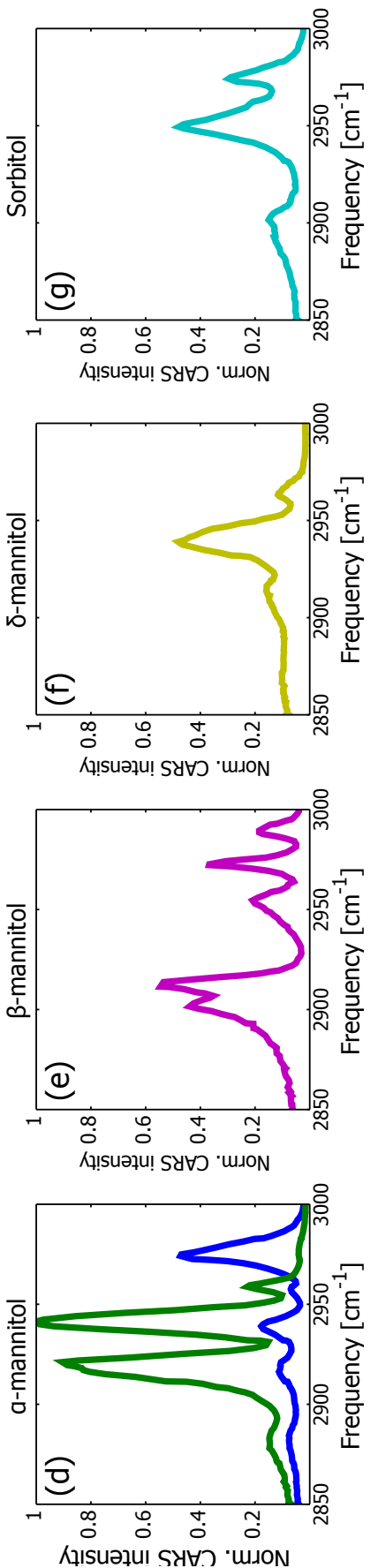

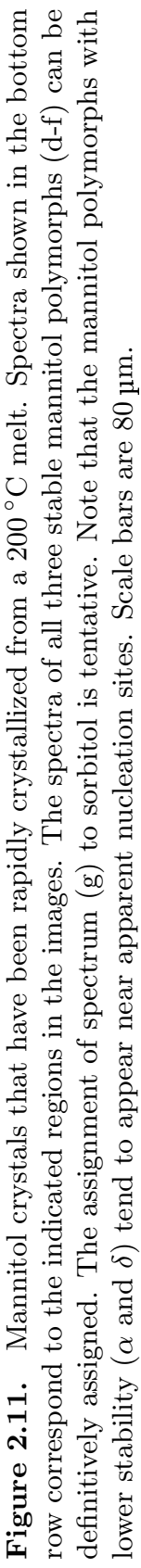


crystal packings; mannitol is reported to have at least three stable polymorphs, one unstable hemihydrate crystal form, and an unstable amorphous form[101103]. Following the conventions of Xie et al.[104] we refer to the three stable polymorphs, in descending order of thermodynamic stability, as the $\beta$, $\alpha$, and $\delta$ forms. Each of these three crystal forms is reported to have a different vibrational spectrum for each orthogonal orientation of the crystal lattice. As a result, a sample of mannitol at ambient conditions can contain up to nine independent spectral bases.

The report of Beattie et al.[105] demonstrates that the orientation of the mannitol crystal with respect to the driving laser fields only influences the relative intensities of the spectral peaks for that polymorph, and does not shift the frequencies of the peak locations. However, the locations of the spectral peaks - particularly in the high-frequency alkyl region - change dramatically as a function of polymorph.

Our sample of mannitol is prepared by quench-cooling an aliquot of $98 \%$ purity $\beta$-mannitol that was heated to $200{ }^{\circ} \mathrm{C}$. The rapid cooling precipitated the thermodynamically quasi-stable $\delta$ form first, followed by the $\alpha$ and $\beta$ forms as the heat transfer slowed. The three projections from a single hyperspectral CARS image are shown in Figs. 2.11(a)-(c). Many different colors indicate a high degree of polymorphism and orientational anisotropy, which is confirmed by the extraction of spectra from regions of high hue contrast. Five independent spectra are easily discovered. As expected, the least stable $\delta$ polymorph appears near a nucleation site, indicated by the yellow arrows in the projection images and the yellow spectrum in Fig. 2.11(f). The $\alpha$ form dominates the majority of the image, appearing in two distinct orientations: the cyan and magenta regions of Fig. 2.11(a). The spectra of two of these regions are plotted in Fig. 2.11(d). As is characteristic for mannitol, these two spectra are composed of vibrations with similar frequencies but different amplitudes. The final polymorph, the $\beta$ form, is present only as a minority component, and is identified by the brilliant cyan color in Fig. 2.11(b). The spectrum from this region agrees well with that measured by Xie[104]. Surprisingly, there is a fifth component in the image, indicated by the cyan arrow and spectrum (Fig. 2.11(g)) whose vibrational spectrum does not agree with any orientation of the known mannitol polymorphs. We have tentatively assigned this spectrum to the compound sorbitol, a stereoisomer of, and common impurity in, mannitol[106, 107]. 


\section{Oral dosage form}

Pharmaceutical oral dosage forms experience a wide variety of chemical and physical processing steps during their manufacture. The number of compounds included in such a formulation can be quite high, and the effect of this processing on the physical forms of the individual materials is not always well characterized, letalone in the presence of the other materials. The number of independent spectra, the random orientations of the components, and the unknown crystal forms combine to create a sample that is challenging to analyze.

The model oral dosage form used in this measurement is composed of the three compounds listed previously - tristearin (50\%), diprophylline (45\%), and $\beta$-mannitol (5\%) - which were mixed, extruded at elevated temperature, and compressed in a tabletting press. Individual particles of all three compounds were initially homogenized to range between $50 \mu \mathrm{m}$ and $80 \mu \mathrm{m}$. The hyperspectral CARS projections of this tablet are shown in Fig. 2.12(a)-(c). The frequencies below $2900 \mathrm{~cm}^{-1}$ were excluded because they are dominated by the tristearin $2845 \mathrm{~cm}^{-1}$ and $2880 \mathrm{~cm}^{-1}$ resonances, the inclusion of which would reduce the dynamic range of the measurements at the frequencies where polymorphisms and orientation effects are most apparent for mannitol and diprophylline. It is immediately apparent from visual analysis of the projections that this sample appears to contain more than three individual components.

Operating by visual analysis, we easily identify the tristearin, which forms the majority of the image and is spatially featureless and spectrally uniform in this frequency region (spectrum shown in Fig. 2.12(f)). Large crystals of diprophylline, which make up slightly less than half of the total volume of the tablet, are likewise easy to select in their vertical and horizontal orientations. Although none of the large diprophylline crystals are oriented precisely along the Cartesian axes of the image, the rotations are in some cases small enough that the spectra do not shift significantly, so that spectral agreement between these crystals and those measured in Figs. 2.9 remains good.

Three other crystals are easy to distinguish based solely on visual analysis. The salmon-colored round object on the bottom-right side of the first projection has a representative $X$-oriented $\beta$-mannitol spectrum. A slightly larger, pale yellow crystal in the top-left corner of the image is also $\beta$-mannitol, though with its crystal axis oriented vertically along the image as can be seen from the relative intensity differences in the four prominent peaks. The third orientation of $\beta$-mannitol is found in the top-center and exact center of the image; it is most easily noticed in Fig. 2.12(b) as a pair of dark red features. 

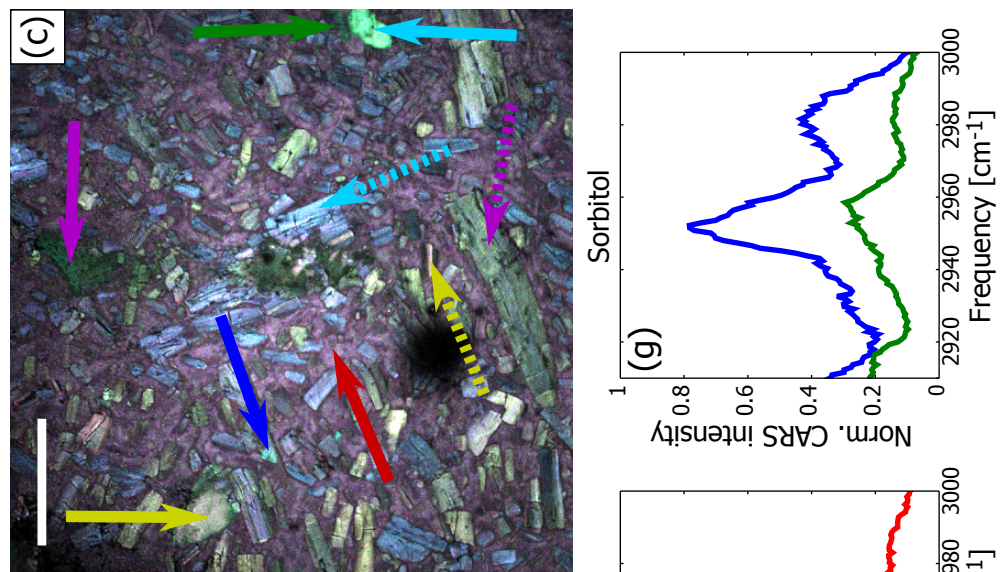

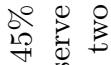

की

寻官

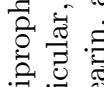

氜.

ㅇํㅇㅛㅛ

드

पे है

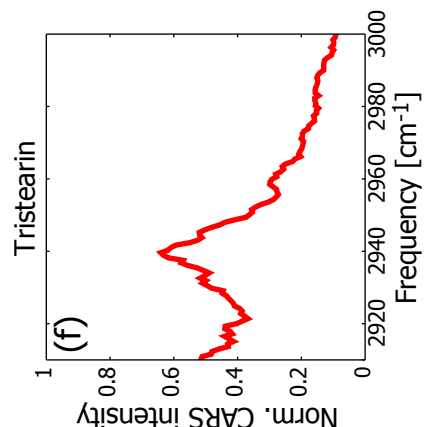

0 \&

융 궁

हี

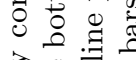

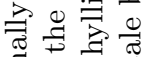

$\exists . \Xi$ कृ

हु दै

잉

텽 음

으응

के चี

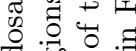

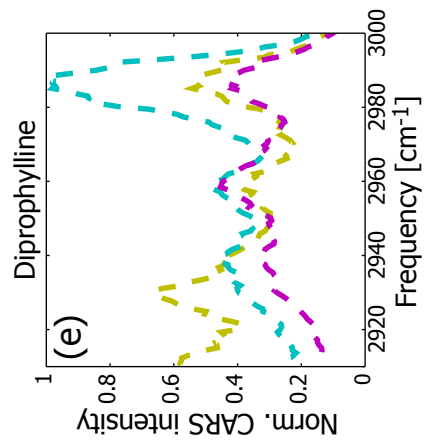

¿
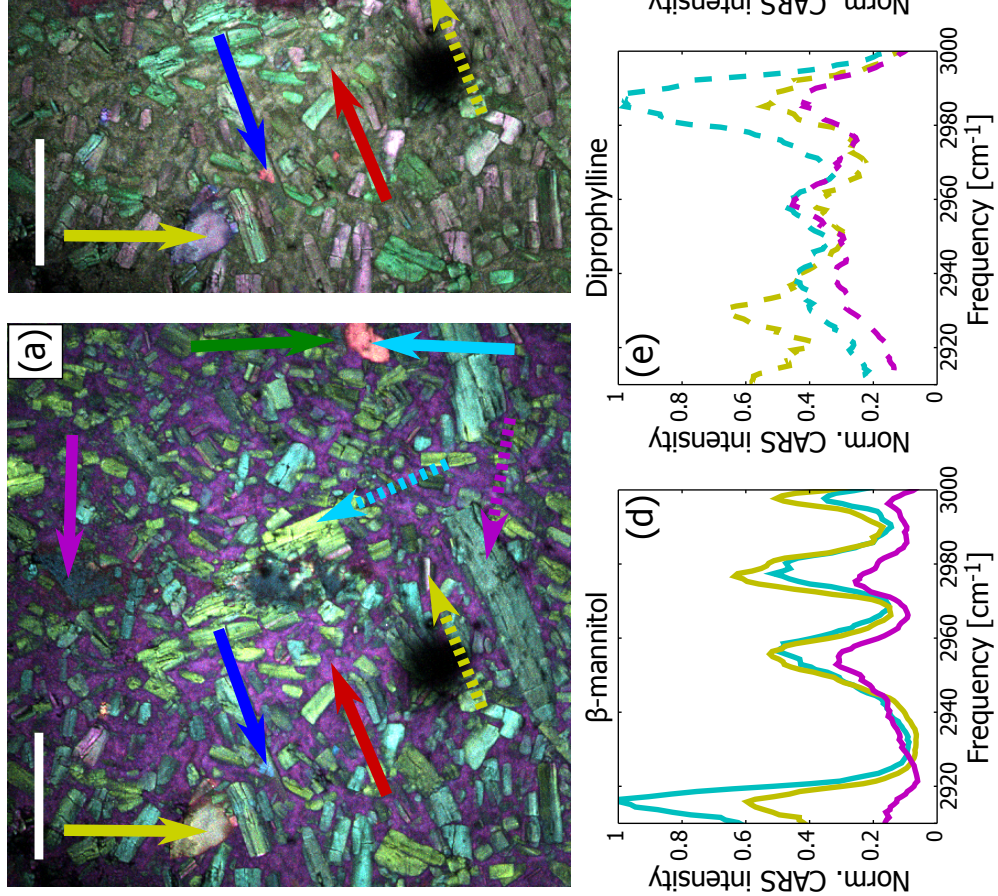

政

๘

䏓

.

車要

융 (2)

तี

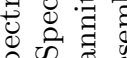

级茟

कें

․․ㄹ

I

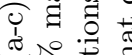

ฮำ

ง 릉

ำ สู

ง ฐٔ क

๑)

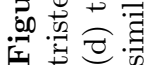


Close examination of the images reveals two additional features, each with linear dimensions of only a few microns. A bright red triangle on the left of Fig. 2.12(b), indicated with the solid blue arrow, is spectrally similar to the feature that was classified as sorbitol in Figs. 2.11. Perhaps more intriguing is a very small object on the extreme right of the image, indicated with green arrows. The spectrum of this object is shifted about $5 \mathrm{~cm}^{-1}$ relative to the blue spectrum, but otherwise overlaps well.

The manufacturing process of solid dosage forms can contain extreme conditions, including high pressures and temperatures and mechanical shearing. In our analysis of the hyperspectral CARS images we found that the dimensions of both diprophylline and mannitol were considerably reduced, and we could not find individual particles of tristearin. These effects are understandable when the manufacturing conditions are considered: the extruder is actively heated close to the melting point of tristearin, which is a soft and malleable material even at room temperature, so fusing of individual tristearin particles during the extrusion is expected. Shearing and grinding of the diprophylline and mannitol crystals during the extrusion and compaction steps break them apart, though the size distribution remains significantly heterogeneous. It is also apparent that there is no preferential orientation for either of these materials after compaction: we have correlated polarization-resolved $\beta$-mannitol Raman spectra for all three crystal orientations to our hyperspectral CARS data, and we can visually determine that all orientations of diprophylline crystals are represented. We have additionally detected at least one impurity in the tablet, though conclusive identification is all but impossible given the nonlinear nature of CARS and the relatively narrow spectral window that we have measured.

\subsubsection{In planta imaging of $\Delta^{9}$-tetrahydrocannabinol}

There has been increasing interest recently in elucidating the mechanisms and pathways by which Nature is able to efficiently produce medicinally useful compounds. Modern drug discovery protocols are expensive and time-consuming, and yield products which can have serious side effects and contraindications. Pharmaceutical research groups take many cues from naturally-occurring compounds in both synthesis and molecular structure[108, 109], but industrial synthetic methods still generally lag far behind Nature in efficiency[110]. New research has also indicated that the efficacy of many natural medicinal compounds is synergistically enhanced by other compounds present in the sample[111]. 
One particularly well-known example of a plant with medicinal benefits is Cannabis sativa $L$. The primary active component, $\Delta^{9}$-tetrahydrocannabinol (THC), is a powerful analgesic and antiemetic agent[112]. In some strains of the Cannabis plant the local concentrations of THC and its precursor, $\Delta^{9}$ tetrahydrocannabinolic acid (THCa), can reach up to 25\%[113]. The presence and ratios of minority cannabinoids, such as cannabinol (CBN) and cannabidiol (CBD), can significantly alter the physiological and psychological effects of consuming the plant. The exact quantities and ratios of the various cannabinoids have been determined with multiple methods[114] and as functions of numerous growth conditions[113]. The high concentrations of active compound makes Cannabis an attractive target for the demonstration of label-free nonlinear imaging on pharmaceutically-relevant materials in planta.

Experimental requirements for the nonlinear optical imaging of plants are significantly different than for animal samples[115, 116]. Many plant cells contain high concentrations of efficient light-absorbing molecules, such as chlorophyll, that limit the penetration of light into the sample and substantially reduce the optical damage threshold compared to transparent animal cells. Further complicating matters is that the fluorescent emissions from botanical light-harvesting complexes tend to spectrally overlap with the anti-Stokes wavelength over a broad range of vibrational frequencies. The fluorescent emissions from these chromophores can overwhelm nonlinear vibrational signals because they are mediated by a lower-order nonlinear optical susceptibility with a substantially higher interaction cross-section.

The majority of the chromophores in Cannabis plants are chlorophyll $a$ and $b$, which have absorption bands at $440 \mathrm{~nm}$ and $450 \mathrm{~nm}$, respectively[117, 118]. The absorption profile of chlorophyll $a$ drops sharply above $440 \mathrm{~nm}$, and is effectively zero above $455 \mathrm{~nm}$, while the tail of the chlorophyll $b$ absorption curve does not reach zero until about $480 \mathrm{~nm}$. Both chlorophyll species fluoresce around $670 \mathrm{~nm}$, with tails that extend below $650 \mathrm{~nm}$.

Absorption bands of both chlorophyll types can be easily excited via twophoton absorption of the OPO signal beam (nominally at $820 \mathrm{~nm}$ ), but neither can be efficiently accessed with a two-photon transition involving the laser fundamental at $1064 \mathrm{~nm}$, and only chlorophyll $b$ can be excited by a sumfrequency transition involving both beams. The only available CARS pathways utilizing these two beams, which probes vibrations in the high-frequency alkyl region, requires both the signal and the laser fundamental beams. An amplitude modulation on the fundamental beam will be fully transferred to the anti-Stokes emission and any fluorescence from chlorophyll $b$, but will not appear on the 
chlorophyll $a$ emission.

To partially overcome the fluorescence of native chromophores we introduced a modulated detection scheme using the OPO signal as the degenerate pump/probe field and the laser fundamental as the Stokes field. The Stokes beam was amplitude-modulated at $2.4 \mathrm{MHz}$ with an acousto-optic modulator (AOM, Model 3080, Crystal Technologies), and the electrical output of the detector was fed to a high-frequency lock-in amplifier (HF2LI, Zurich Instruments) set to demodulate in phase with the Stokes modulation. Two-photon absorptions of the OPO signal beam appear as a DC signal to the lock-in, and so are effectively discarded. The modulation frequency is significantly higher than the pixel update rate, so aliasing is avoided.

Highly pure (>97\%) THCa (measured by ${ }^{1} \mathrm{H}-\mathrm{NMR}$ and HPLC) were obtained from Sigma Aldrich (39382-10MG) and imaged without further processing. Reference spectra from these crystals were used to identify cannabinoidrich regions in the plants. Dried samples of female Cannabis sativa L., consisting primarily of pistillate flowers and adjacent bracts, were obtained from a local distributor in Enschede, the Netherlands. White-light stereomicroscopy was used to verify the presence, density, and integrity of glandular trichomes, and to identify structures of interest for nonlinear imaging. Small sections of the plants were carefully removed and immersed in deionized water between two cover-glasses for imaging. Hyperspectral CARS reference images of the THCa were recorded using the signal-idler beam combination for increased spectral bandwidth, while all plant imaging was performed with the signal-fundamental combination. The total average power on the plant sections during imaging was kept below $100 \mathrm{~mW}$ to avoid sample damage.

\section{$\Delta^{9}$-tetrahydrocannabinolic acid}

An example THCa reference image, consisting of $256 \times 256$ pixels over 117 spectral points and recorded in $120 \mathrm{~s}$ in transmission, is shown in Fig. 2.13(a), with the spectra from the indicated regions plotted in Fig. 2.13(b). Of particular note is that there are two distinct spectra that we label $\mathrm{THC}_{1}$ and $\mathrm{THC}_{2}$, each of which appears in about $50 \%$ of the crystals we imaged. The $\mathrm{THC}_{1}$ alkyl spectrum is complicated and congested - to be expected given the structure of the THCa molecule, with $\mathrm{CH}_{2}$ and $\mathrm{CH}_{3}$ groups in numerous functional groupswith distinct peaks at $2825 \mathrm{~cm}^{-1}, 2868 \mathrm{~cm}^{-1}, 2907 \mathrm{~cm}^{-1}$, and $2940 \mathrm{~cm}^{-1}$, while the $\mathrm{THC}_{2}$ spectrum has a single prominent feature at $2903 \mathrm{~cm}^{-1}$ and a minor secondary peak at $2960 \mathrm{~cm}^{-1}$. We verified that both spectra are insensitive to 
the orientation of crystals relative to the laser fields, and they appear identical under both white-light reflection and transmission. The spectral features in both cases are narrow and prominent, indicating a high degree of crystallinity. As the purity of the THCa is guaranteed to $>97 \%$ via analytical methods, we therefore propose that these two spectra represent as-yet un-reported polymorphs of THCa.

\section{Cannabis sativa L. leaf}

Nonlinear imaging of Cannabis leaves was performed in epi-detection; the strong absorption of the anti-Stokes wavelength by chlorophyll in the thick samples precluded detection of the forward-scattered signal. The presence of numerous fluorescent bodies within the plants necessitated modulated detection. A representative hyperspectral CARS image of a flowering bract, consisting of $512 \times 512$ pixels recorded over 80 spectral points at 7 seconds per frame, is shown in Fig. 2.14(a). Spectra extracted from two regions are shown in Fig. 2.14(b). The flat, featureless spectrum (green curve) is characteristic of fluorescence, while the blue trace matches closely to $\mathrm{THC}_{1}$. We believe that the fluorescence originates from chlorophyll $b$, which can be excited by a twophoton process involving both the OPO signal and the laser fundamental, and whose emission is therefore modulated.

The physical morphology of the electronic and vibrational resonant regions are also dissimilar. All of the vibrational signal comes from a single diffuse, oblong region that is tens of microns in diameter. The spatial features of this region are consistent with a storage reservoir or vesicle, with small irregularities at the edges possibly indicating a rupture or derangement of the walls of the structure. The lack of spectral features aside from the characteristic $\mathrm{THC}_{1}$ spectrum indicate that the contents of this structure are relatively homogeneous and pure, while the smooth spatial appearance indicates that these contents are in a disordered phase. Whether this phase is solvated, amorphous, or nanocrystalline is an open question. All of these characteristics differ significantly from the fluorescent regions, which are small, compact, and heterogeneous.

\section{Cannabis sativa L. pistil}

The pistils of flowering female Cannabis sativa L. plants are known to contain large quantities of cannabinoids, with average concentrations of 10-12\%/w[119]. We have observed that, in contrast to the leaves, the pistils of Cannabis flowers 

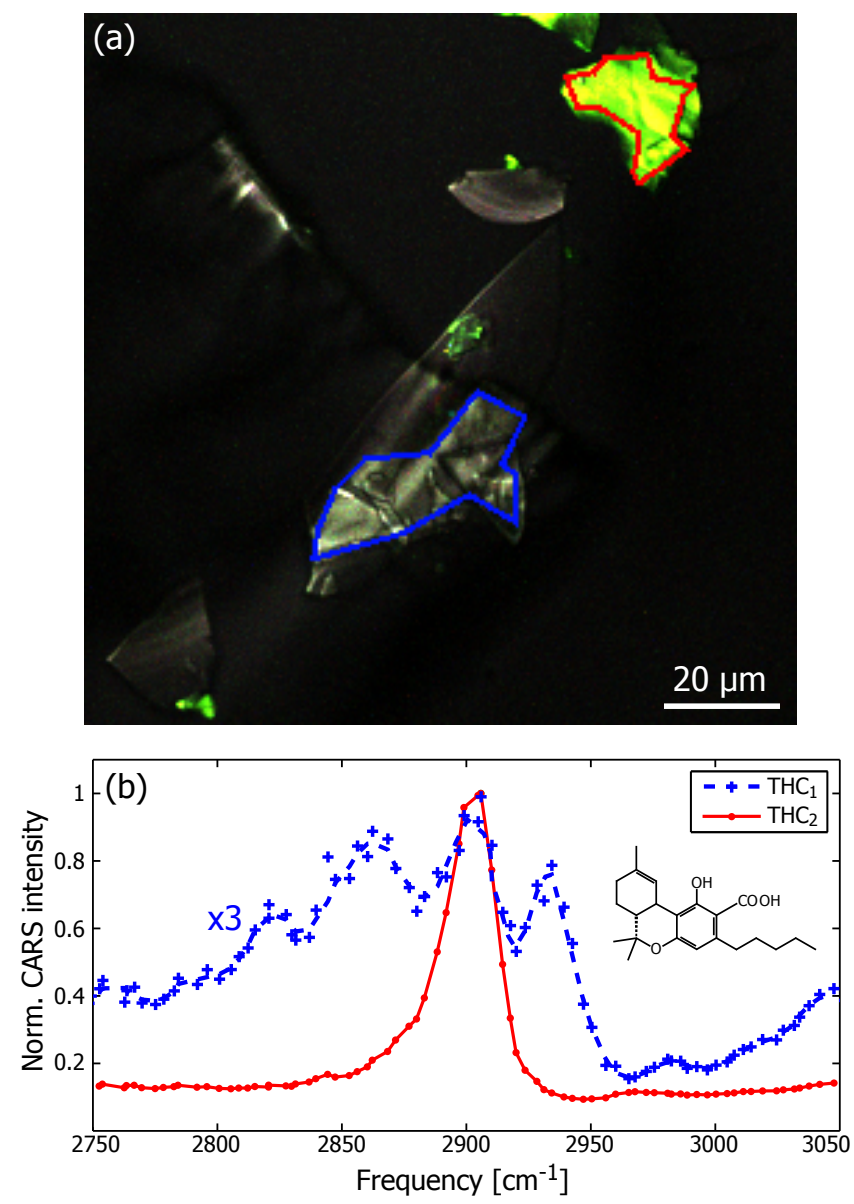

Figure 2.13. (a) Hyperspectral CARS image of pure THCa crystals displaying two distinct colors. (b) Spectra extracted from the indicated regions of (a). Key marker frequencies for $\mathrm{THC}_{1}$ are $2825 \mathrm{~cm}^{-1}, 2860 \mathrm{~cm}^{-1}, 2905 \mathrm{~cm}^{-1}, 2940 \mathrm{~cm}^{-1}$, while $\mathrm{THC}_{2}$ has only a single strong peak at $2900 \mathrm{~cm}^{-1}$. Small inclusions of $\mathrm{THC}_{2}$ within the $\mathrm{THC}_{1}$ region generate additional intensity at $2900 \mathrm{~cm}^{-1}$ that appears to shift the $\mathrm{THC}_{1}$ peak lower. The THCa molecular structure is shown in part (b). Note that both spectra in part (b) have been globally normalized to the maximum of the $\mathrm{THC}_{2}$ peak, and that the $\mathrm{THC}_{1}$ spectrum has been additionally scaled up by a factor of three.

contain dense regions of highly concentrated cannabinoids in complicated and varied structures. Because the pistils are fairly thin and nearly transparent, CARS detection in transmission is utilized. This confers an advantage because 

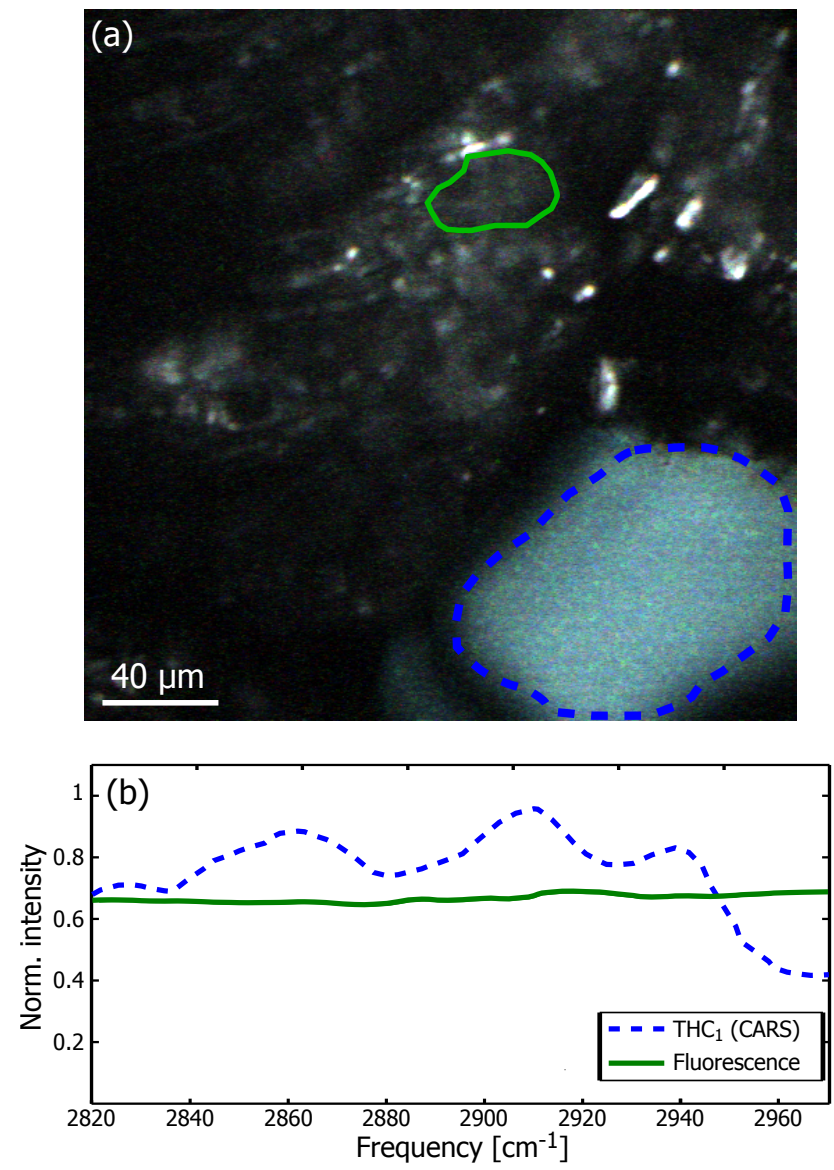

Figure 2.14. Hyperspectral CARS image (a) and spectra (b) from a Cannabis sativa $L$. leaf, recorded in reflection, showing native fluorescence (green region and curve) and a $\mathrm{THC}_{1}$ CARS signal (blue region and curve).

the CARS emissions are highly directional in a narrow cone due to phasematching, enabling efficient collection and relaying of the anti-Stokes light to detectors.

Most of the $\mathrm{THC}_{1}$ emissions that we have recorded in planta from the pistils appear to originate in structures with linear dimensions greater than $1 \mu \mathrm{m}$, as illustrated by the $z$-stack projection of a pistillate trichome Fig. 2.15(a). To create this image the laser focus was axially translated by $1 \mu \mathrm{m}$ through the sample between individual frames. A total of 60 frames were recorded at a 

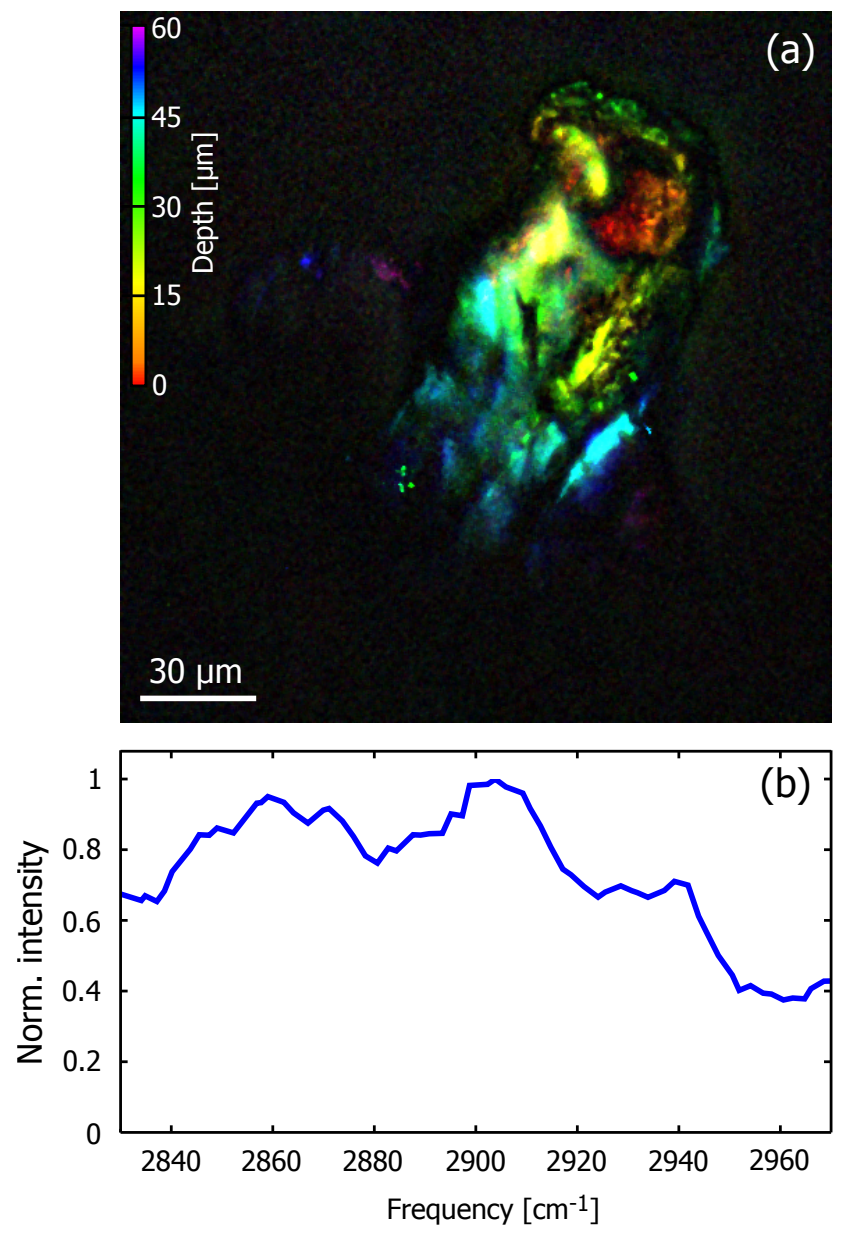

Figure 2.15. (a) Color-coded projection of a CARS z-stack in a glandular trichome, measured at the $\mathrm{THC}_{1}$ frequency of $2940 \mathrm{~cm}^{-1}$. Reds and oranges indicate the bottom of the stack, while blues and purples are at the top of the stack. Many different physical morphologies are visible at different regions within this trichome. (b) CARS spectrum extracted from a slice in the center of the stack. It agrees well with the reference $\mathrm{THC}_{1}$ spectrum.

constant vibrational frequency of $2940 \mathrm{~cm}^{-1}$. Each frame was recorded in 10 seconds, averaged three times. We verified that the bright signals correspond to a $\mathrm{THC}_{1}$ CARS signals by taking a hyperspectral image (Fig. 2.15(b)) at a slice in the center of the stack. To depict the three-dimensional structure of the entire trichome we have applied a z-dependent color to each slice (from the 
Z-Stack functions of the MBF library in ImageJ). The hazy background in the top half of the image is the non-resonant background of the water immersion medium. Reds and yellows indicate regions towards the bottom of the stack, while blues and purples are at the top of the stack. There are four distinct regions that can be identified. The red/orange section at top-right is highly structured and dense, and contains small voids or vacuoles. Directly beneath that section are two unconnected regions of similar morphology at different heights in the sample, colored yellow/green and light blue, respectively. These regions have sharp boundaries and are elongated and narrow. The left side of the trichome displays a large and more diffuse distribution without clear and sharp boundaries (yellow, green, light blue), similar to that observed in the bract. Finally, the far top-right of the trichome has a number of very thin regions of cannabinoids, shown in green, that may be closely associated with or bound to cell membranes. From these images the compartmentalized distribution of the cannabinoids is readily apparent. The observation of these different morphologies has not, to the best of our knowledge, been previously reported.

We have also imaged a region within an intact stigma of a female flower that contains both $\mathrm{THC}_{1}$ and $\mathrm{THC}_{2}$ in close proximity. The hyperspectral CARS image shown in Fig. 2.16(a) was recorded at a depth of about $30 \mu \mathrm{m}$, with $512 \times 512 \times 80$ pixels at 10 seconds per frame. The spectra from the magenta and blue regions of the image correspond approximately to $\mathrm{THC}_{1}$ and $\mathrm{THC}_{2}$, respectively, and are shown in Fig. 2.16(b). Additionally, a small volume of a native crystalline lipid, which is easily identified by its characteristic $\mathrm{CH}_{2}$ symmetric stretch at $2845 \mathrm{~cm}^{-1}$ and $\mathrm{CH}_{2}$ asymmetric stretch at $2880 \mathrm{~cm}^{-1}$ [100], is outlined in green. Deviations from the reference spectrum of $\mathrm{THC}_{1}$ can be attributed to the presence of other molecules in the focal volume of the laser beam, including other cannabinoids such as cannabinol and cannabidiol[112]. The broadening of the $\mathrm{THC}_{2}$ peak is a result of detector saturation, a side effect of increasing the contrast of other regions in the image. At non-saturating gain settings the spectrum is nearly identical to that shown in Fig. 2.13 for $\mathrm{THC}_{2}$. 

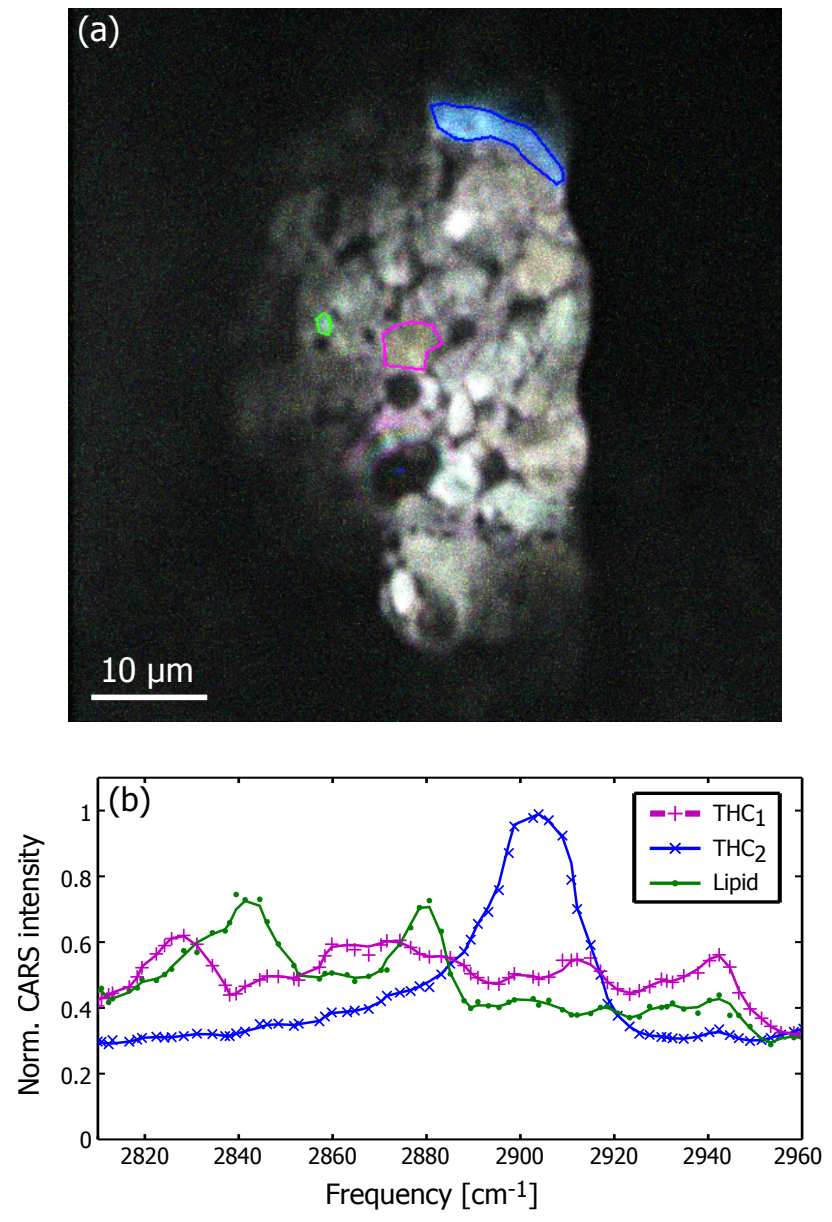

Figure 2.16. Hyperspectral CARS image (a) and spectra (b) of the interior of a stigma containing both $\mathrm{THC}_{1}$ (magenta) and $\mathrm{THC}_{2}$ (blue) in close proximity. Additionally, a small, native lipid droplet is visible (green), with a peak at $2880 \mathrm{~cm}^{-1}$ indicating significant crystallinity. 



\section{CHAPTER 3}

\section{Vibrational phase contrast CARS}

Hyperspectral CARS is a powerful tool for obtaining qualitative information about samples. The mixing of $\chi_{R}^{(3)}$ and $\chi_{N R}^{(3)}$ is difficult to disentangle, and it is a challenge to linearize the nonlinear dependence of the CARS intensity on the number of oscillators in the focal volume. While computational methods have been developed to retrieve $\chi^{(3)}$ from hyperspectral (or broadband) CARS data[93, 95, 120], they cannot operate on single-frequency images. Measuring the complex $\chi^{(3)}$ on a pixel-by-pixel basis is inherently faster than taking full spectra at every pixel and disentangling the data, and so can be used to monitor dynamic samples (e.g. fluid mixing).

\subsection{Theory}

\subsubsection{The molecular rollercoaster}

A concept developed by Lucassen et al. [15] (the circle method) and expanded upon by Jurna and Garbacik et al.[21] (as the molecular rollercoaster) is convenient for representing vibrational resonances. The complex $\chi^{(3)}$ can be separated into real and imaginary parts,

$$
\chi^{(3)}=\chi_{R}^{(3)}+\chi_{N R}^{(3)}=\left(\Im\left[\chi_{R}^{(3)}\right]\right)+\left(\Re\left[\chi_{R}^{(3)}\right]+\chi_{N R}^{(3)}\right)
$$

where the resonant terms have the forms

$$
\begin{aligned}
& \Im\left[\chi_{R}^{(3)}\right]=\sum_{n} \frac{A_{n}}{1+\Delta_{n}^{2}} \\
& \Re\left[\chi_{R}^{(3)}\right]=\sum_{n} \frac{A_{n} \Delta_{n}}{1+\Delta_{n}^{2}}
\end{aligned}
$$

and we have defined $\Delta_{n}=\left(\omega_{p}-\omega_{s}-\Omega_{n}\right) / \Gamma_{n}$ for the vibrational mode at natural frequency $\Omega_{n}$ and with spectral bandwidth $\Gamma_{n}$. The complex Cartesian components of $\chi^{(3)}$ can also be represented in polar coordinates. The position 
vector representing $\chi^{(3)}$ in the complex plane has a magnitude given by the length of the vector and an phase defined to be the angle between the vector and the real axis

$$
\begin{aligned}
& A_{\chi^{(3)}}=\sqrt{\Im\left[\chi_{R}^{(3)}\right]^{2}+\left(\Re\left[\chi_{R}^{(3)}\right]+\chi_{N R}^{(3)}\right)^{2}} \\
& \phi_{\chi^{(3)}}=\tan ^{-1}\left(\frac{\Im\left[\chi_{R}^{(3)}\right]}{\Re\left[\chi_{R}^{(3)}\right]+\chi_{N R}^{(3)}}\right)
\end{aligned}
$$

A single isolated resonance will trace a circle in the complex plane when plotted parametrically as a function of driving frequency, with the non-resonant background merely shifting the circle along the real axis. Multiple overlapping resonances will generate additional loops in the complex plane. When the real and imaginary components of $\chi^{(3)}$ are plotted against frequency in complex space, the resulting trajectory forms a spiraling molecular rollercoaster. An example of the complex spectra of two compounds, along with individual plots of their Cartesian and polar components, is shown in Figs. 3.1. Note that the phase runs from 0 to $-\pi$; this is a convention that represents an emission process.

Combinations of two or more compounds can be analyzed within this formalism. The axes of the complex plane form an orthogonal basis, so the complex response $\chi^{(3)}=X+i Y$ of a mixture of weakly-interacting compounds is simply represented as the linear combination of their individual complex responses $\left(x_{i}, y_{i}\right)$ multiplied by their volume fractions $\rho_{i}$,

$$
\begin{aligned}
& X(\omega)=\sum_{i} \rho_{i} x_{i}(\omega)+\chi_{N R}^{(3)} \\
& Y(\omega)=\sum_{i} \rho_{i} y_{i}(\omega)
\end{aligned}
$$

with the condition that $\sum_{i} \rho_{i}=1$. This relationship breaks down if the compounds strongly interact with each other. For example, the complex vibrational response of a mixture of equal parts water and ethanol, which form hydrogen bonds, cannot be represented as the linear combination of the pure water and ethanol resonances[21]. 

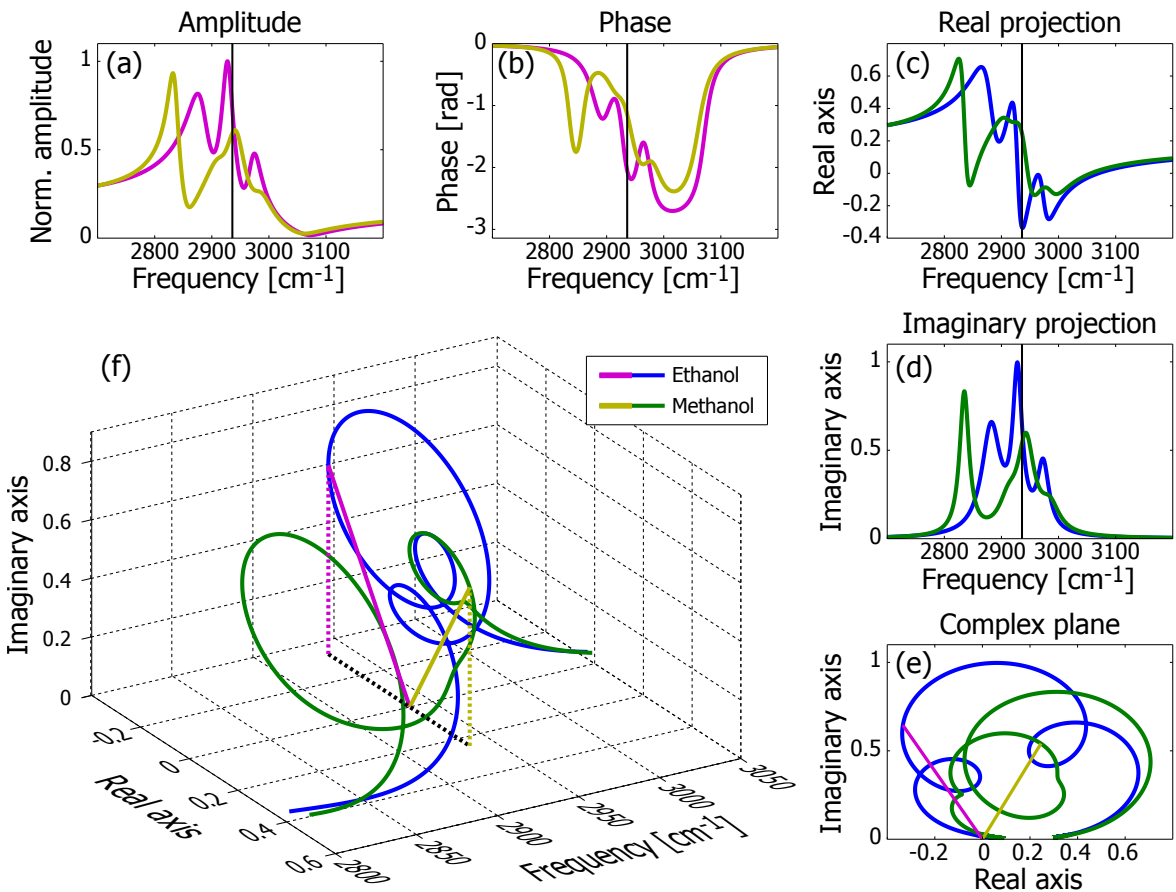

Figure 3.1. When plotted against the driving frequency, the amplitude (a) and phase (b) of the response of an oscillator form a spiraling trajectory through complex space: (f) the molecular rollercoaster. The projections of this trajectory along each Cartesian axis yield valuable information about the oscillatory response. The projections yield the real component (c, projection along imaginary axis), the imaginary component (d, projection along real axis), and the complex plane (e, projection along frequency axis). Conversely, knowledge about any two plots (a-e) allows construction of the rollercoaster and subsequent extraction of the other three components.

\subsubsection{Heterodyne phase detection}

Detecting the amplitude and phase of the molecular response in every spatial pixel was first demonstrated by Potma et al.[13]. In a narrowband heterodyne CARS experiment a local oscillator that is phase-locked to the anti-Stokes field is applied to the sample collinearly and temporally overlapped with the pump and Stokes fields, then collected and directed towards a detector with high 
dynamic range. The intensity of the signal on the detector is

$$
I_{d e t}=\left|E_{C A R S}+E_{L O}^{*}\right|^{2}=\left|E_{C A R S}\right|^{2}+\left|E_{L O}\right|^{2}+2 E_{C A R S} E_{L O}^{*} \cos \phi
$$

with the fields $E_{C A R S}$ and $E_{L O}^{*}$ given as complex quantities and $\phi$ is the phase angle between the two fields. It is critical to note here that the CARS field contains information about the full complex nonlinear susceptibility $\chi^{(3)}$. Nonlinear processes are inherently very weak, so the detected signal will be dominated by the second term on the right hand side of Eq. 3.5, even when the local oscillator is at nanowatt levels. This term is effectively a DC signal; most CARS experiments utilize a modelocked laser with a repetition rate in the tens of megahertz, which is higher than the bandwidth of many detectors. If the phase difference $\phi$ between the local oscillator and CARS fields carries a time dependence $\phi(t)=2 \pi \nu_{\text {mod }} t$, then the last term in Eq. 3.5 will oscillate with frequency $\omega_{\text {mod }}=2 \pi \nu_{\text {mod }}$. This AC signal can be easily separated from the large DC background of the local oscillator and analyzed with conventional lock-in techniques. The phase of the CARS field can be determined from this measurement, and because the field amplitude is measured the signal scales linearly with the number of oscillators in the focal volume.

In theory heterodyne CARS is an ideal tool to exactly measure the antiStokes field. In practice it is experimentally challenging to keep the local oscillator phase-locked to the CARS field. Thermal drift of the optical setup, phase variations across the field of the view of the microscope objective, and mechanical vibrations all affect the path length between the locations where the local oscillator and anti-Stokes fields are generated. Measuring this local phase is critical if the pure vibrational phases of the oscillators in the sample are to be determined.

\subsubsection{Homodyne phase detection}

Jurna et al. developed a method for measuring this local phase, which they called vibrational phase contrast CARS, or VPC-CARS for short. In VPCCARS the heterodyne CARS signal is measured as described above, with a local oscillator interfering with an anti-Stokes field at a detector. As originally described by Jurna et al., the local phase is detected separately as the homodyne interference between two separate CARS pathways in the same vibrational level. In this experiment three pump fields are generated in a cascaded phase-preserving chain (CPPC) centered around a frequency-doubled 


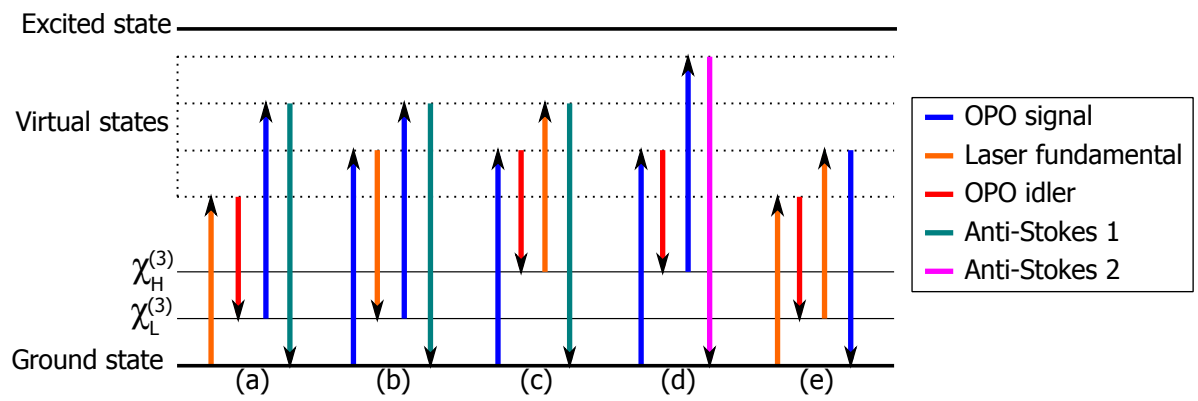

Figure 3.2. $\quad$ Energy level diagrams for the five relevant CARS processes. Paths (a), (b), and (c) are detected on a photomultiplier tube. Path (e) is heterodyne detected on a photodiode. Note that path (d) is not directly detected due to its short emission wavelength, but is included for completeness.

modelocked pump laser (producing a beam with nominal carrier frequency $\omega_{f}$, doubled to $\omega_{P}=2 \omega_{f}$ ) which drives an OPO (yielding signal and idler beams according to $\left.2 \omega_{f}=\omega_{s}+\omega_{i}\right)$. The residual laser fundamental beam is passed through an acousto-optic modulator where frequency sidebands at $\delta \omega$ are applied, yielding a beam with carrier frequency $\omega_{F+}=\omega_{f}+\delta \omega_{f}$.

At this point we introduce the shorthand notation

$$
\begin{aligned}
S & =A_{s} e^{-i\left(\omega_{s} t+\phi_{s}\right)} \\
F & =A_{f} e^{-i\left(\left(\omega_{f}+\delta \omega_{f}\right) t+\phi_{f}\right)} \\
I & =A_{i} e^{-i\left(\omega_{i} t+\phi_{i}\right)},
\end{aligned}
$$

for the three pump fields. The terms $\phi_{n}$ for each field are assumed to vary slowly and represent the cumulative local phase offsets in the experiment. When the modulated fundamental is combined with the signal and idler beams at the sample, the resulting interaction yields five possible CARS pathways. The nonlinear material polarization generated by this set of fields, reproduced from Eq. 2.2 with the relevant fields, contains five terms, namely

$$
\begin{aligned}
P^{(3)} & \propto \chi_{L}^{(3)}\left[F I^{*} S+S F^{*} S+F I^{*} F\right]+c . c . \\
& +\chi_{H}^{(3)}\left[S I^{*} S+S I^{*} F\right]+c . c .
\end{aligned}
$$

where $N^{*}$ indicates the complex conjugate of field $N$. These five pathways all 
probe the vibrational frequencies

$$
\begin{aligned}
& \Delta \omega_{L}=\omega_{s}-\omega_{f}=\omega_{f}-\omega_{i} \\
& \Delta \omega_{H}=\omega_{s}-\omega_{i}=2 \Delta \omega_{L}
\end{aligned}
$$

because of energy conservation resulting from the CPPC $\left(2 \omega_{f}=\omega_{s}+\omega_{i}\right)$. These optical pathways are shown in Figs. 3.2, where $\chi_{L}^{(3)}$ and $\chi_{H}^{(3)}$ represent the local complex nonlinear susceptibilities in each of two vibrational manifolds. This notation is a matter of book-keeping, since the entire molecular response is represented by a single $\chi^{(3)}$.

The anti-Stokes emissions from the first three pathways depicted in Figs. 3.2 are detected as an intensity on a single photomultiplier tube. This intensity is the square modulus of the anti-Stokes field generated by the material polarization in Eq. $3.7^{1}$ :

$$
\begin{aligned}
I_{L O C}=\left|P^{(3)}\right|^{2} & \propto\left|\chi_{L}^{(3)}\right|^{2}\left[\left|S^{2} F\right|^{2}+|F I S|^{2}+\left|F^{2} I\right|^{2}\right] \\
& +\left|\chi_{L}^{(3)}\right|^{2}\left[|S|^{2} F^{2} S^{*} I^{*}+\left(F^{*}\right)^{3} S^{2} I^{*}+|F I|^{2} S F^{*}\right]+\text { c.c. } \\
& +\left|\chi_{H}^{(3)}\right|^{2}\left[\left|S^{2} I\right|^{2}+|S I F|^{2}+|S I|^{2} S F^{*}\right]+c . c . \\
& +\chi_{L}^{(3)} \chi_{H}^{*(3)}\left[\left|S^{2}\right|^{2} F^{*} I^{*}+|S|^{2}\left(F^{*}\right)^{2} S I\right]+c . c . \\
& +\chi_{L}^{(3)} \chi_{H}^{*(3)}\left[|S I|^{2} S^{*} F+|F I S|^{2}\right]+\text { c.c. } \\
& +\chi_{L}^{(3)} \chi_{H}^{*(3)}\left[\left(S^{*}\right)^{2}|I|^{2} F^{2}+|F I|^{2} F S^{*}\right]+\text { c.c. }
\end{aligned}
$$

The only terms that are relevant for us are those which contain $F^{2} S^{*} I^{*}$ and its complex conjugate (indicated in red in Eq. 3.9)

$$
\begin{aligned}
F^{2} S^{*} I^{*} & =|F|^{2}|S||I| e^{-i\left(2 \omega_{f}-\omega_{s}-\omega_{i}+2 \delta \omega_{f}\right) t+2 \phi_{f}-\omega_{s}-\omega_{i}} \\
& =|F|^{2}|S||I| e^{-i\left(2 \delta \omega_{f}\right) t+2 \phi_{f}-\omega_{s}-\omega_{i}}
\end{aligned}
$$

The high-frequency terms in the exponent cancel because of the CPPC energy conservation, and the only $\mathrm{AC}$ term remaining in the exponent has a frequency of $2 \delta \omega_{f}$. Lock-in detection allows separation of this $\mathrm{AC}$ term from the other terms in Eq. 3.9 and converts the modulated signal into a real amplitude and

\footnotetext{
${ }^{1}$ We denoted the square modulus of a complex number by $|N|^{2}$, while two complex numbers directly multiplied appear as $N^{2}$ or $\left(N^{*}\right)^{2}$; terms containing $N N N^{*}$ therefore reduce to $|N|^{2} N$, while $N N N$ reduces to $N^{3}$ and $N^{*} N^{*} N^{*}$ to $\left(N^{*}\right)^{3}$.
} 
a slowly-varying phase. The full homodyne demodulated signal is therefore

$$
\begin{aligned}
& I_{L O C}= \chi_{L}^{*(3)}\left(\chi_{L}^{(3)}+\chi_{H}^{(3)}\right)|S|^{2} F^{2} S^{*} I^{*}+c . c . \\
&=\left|\chi_{L}^{(3)}\right|^{2}|S|^{2} F^{2} S^{*} I^{*}+\chi_{L}^{*(3)} \chi_{H}^{(3)}|S|^{2} F^{2} S^{*} I^{*}+c . c \\
&=|S|^{2}\left[\left|\chi_{L}^{(3)}\right|^{2} e^{-i(0)}+\left|\chi_{L}^{(3)} \chi_{H}^{(3)}\right| e^{-i\left(\phi_{H}-\phi_{L}\right)}\right] \\
& \quad \times|F|^{2}|S||I| e^{-i\left(2 \phi_{f}-\phi_{s}-\phi_{i}\right)}+\text { c.c. }
\end{aligned}
$$

We have explicitly left a constant "phase" term at the beginning of the final line for book keeping. This term is constant, and when $\Delta \omega_{H}$ is far off resonance - as is the case when $\Delta \omega_{L} \approx 3000 \mathrm{~cm}^{-1}$ - then the only remaining term is

$$
I_{L O C}=|S|^{2}\left|\chi_{L}^{(3)}\right|^{2}|F|^{2}|S||I| e^{-i\left(2 \phi_{f}-\phi_{s}-\phi_{i}\right)}+\text { c.c. }
$$

The only phase terms left are those of the individual fields, which together constitute a local phase offset. However, in the event that $\Delta \omega_{H}$ is near a resonance, such as when $\Delta \omega_{L} \approx 1450 \mathrm{~cm}^{-1}$, then the amplitude and phase contributions from $\chi_{H}^{(3)}$ come into play and must be taken into account. Because the demodulated signal in this measurement condition contains phase information from at least one resonance, we can no longer refer to it as a strictly local phase. Instead, we will refer to this channel as the homodyne signal, since the signal that we demodulate is the result of two internal pathways beating against each other, and to uniquely distinguish it from the heterodyne channel.

The demodulated heterodyne signal that we measure, reproduced from Eq. 3.5 with the appropriate fields and shown schematically in Fig. 3.2(e), takes the form

$$
\begin{aligned}
I_{H E T} & =\chi_{L}^{(3)}\left[F I^{*} F\right] S^{*}+c . c . \\
& =\chi_{L}^{(3)}\left[F^{2} S^{*} I^{*}\right]+c . c . \\
& =\left|\chi_{L}^{(3)}\right||S|^{2}|F||I| e^{-i\left(2 \phi_{f}-\phi_{s}-\phi_{i}+\phi_{L}\right)}+c . c .
\end{aligned}
$$

Taking the quotient of the demodulated homodyne (Eq. 3.11) and heterodyne 
(Eq. 3.13) channel intensities gives

$$
\begin{aligned}
& I_{L O C} / I_{H E T}= \frac{|S|^{2}\left[\left|\chi_{L}^{(3)}\right|^{2} e^{-i(0)}+\left|\chi_{L}^{(3)} \chi_{H}^{(3)}\right| e^{-i\left(\phi_{H}-\phi_{L}\right)}\right]}{\left|\chi_{L}^{(3)}\right||F|^{2}|S||I| e^{-i\left(2 \phi_{f}-\phi_{s}-\phi_{i}+\phi_{L}\right)}} \\
& \times|F|^{2}|S||I| e^{-i\left(2 \phi_{f}-\phi_{s}-\phi_{i}\right)} \\
&=|S|^{2}\left[\left|\chi_{L}^{(3)}\right| e^{i \phi_{L}}+\left|\chi_{H}^{(3)}\right| e^{-i\left(\phi_{H}-2 \phi_{L}\right)}\right] \\
&=|S|^{2}\left[\chi_{L}^{*(3)}+\chi_{H}^{(3)} e^{i\left(2 \phi_{L}\right)}\right]
\end{aligned}
$$

where the identity $\exp (a+b)=\exp (a) \times \exp (b)$ has been used to combine the phase terms. Note that the last line contains the full complex susceptibilities, not only the magnitudes.

There are many interesting implications from this equation. Of particular note is that the phases of the low- and high-frequency modes have opposite signs; in principle this feature can be used to enhance the chemical specificity of single-frequency VPC-CARS microscopy measurements by opening up the entire complex plane, not just the upper half. This expanded description of the homodyne interference does not contradict the previous phenomenological description by Jurna et al. that explained VPC-CARS signals probing $\Delta \omega_{L} \approx$ $3000 \mathrm{~cm}^{-1}$, the high-frequency alkyl region. One can see that when $\chi_{H}^{(3)}$ is sufficiently off resonance it will have a negligible amplitude and zero phase. The last term in Eq. 3.14 can then be ignored, and $\chi_{L}^{*(3)}$ is recovered. However, when $\Delta \omega_{H}$ is resonant a coupling occurs between the high-frequency and lowfrequency modes. This coupling presents itself most dramatically when there are two resonances $\chi_{L}^{(3)}$ and $\chi_{H}^{(3)}$ such that $2 \Omega_{L} \approx \Omega_{H}$.

We demonstrate the interaction of a pair of resonances as the frequency difference between them changes. In both cases a strong resonance $\chi_{1}^{(3)}$ centered at $\Omega_{1}=0$ with unity amplitude and $\Gamma=10$ interferes with a small resonance $\chi_{2}^{(3)}$ with an order of magnitude lower amplitude and identical natural line width. The non-resonant background is set to $\chi_{N R}^{(3)}=0.2$. To simulate the physical case where $\Delta \Omega_{L} \approx 3000$ we set $\chi_{H}^{(3)}=\chi_{N R}^{(3)}$ and $\chi_{L}^{(3)}=$ $\chi_{1}^{(3)}+\chi_{2}^{(3)}$ and calculate the homodyne intensity for the six values $\Omega_{2}=$ $\{-100,-60,-20,+20,+60,+100\}$. The intensity, phase, Cartesian complex components, complex trajectory, and quotient of the homodyne and heterodyne channels are plotted in Figs. 3.3. Of particular note is that the complex plane has a mirror symmetry about the imaginary axis, indicating that the two 

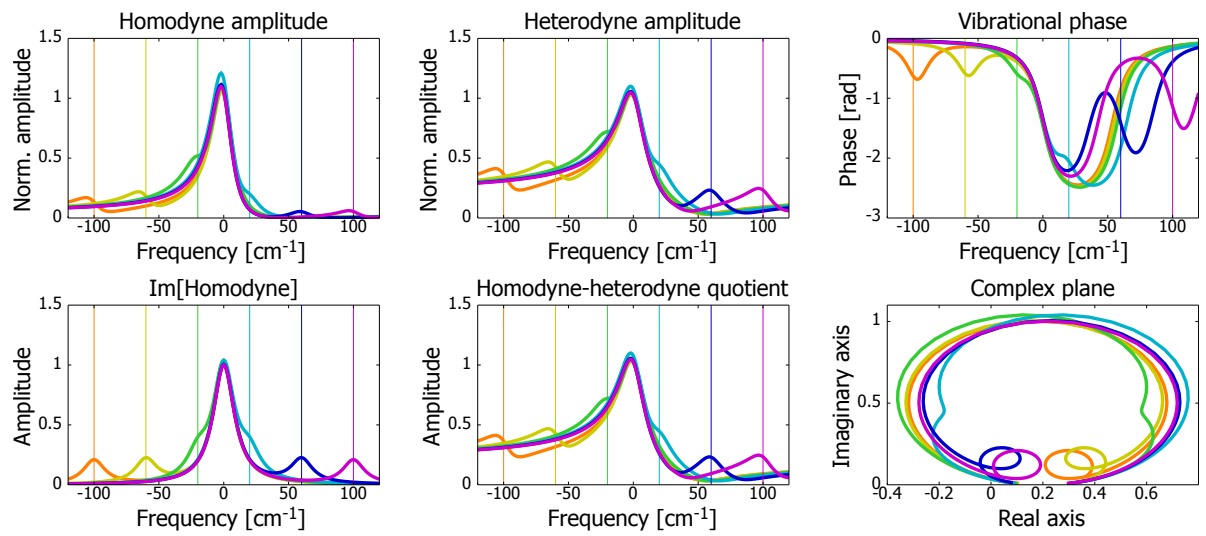

Figure 3.3. Two resonances in the same frequency manifold without the interference of a resonance in the half-frequency manifold. Plotted are (a) the intensity measured on the homodyne detector, (b) the amplitude measured on the heterodyne detector, (c) the vibrational phase, (d) the projection of the imaginary component of the homodyne intensity versus vibrational frequency, (e) the quotient of the local and heterodyne channels, and (f) the complex trajectory. Six curves are plotted, with a principal resonance located at $\Omega_{1}=0$ and a satellite minor resonance at values ranging from $\Omega_{2}=-100$ to $\Omega_{2}=+100$. The vertical colored lines indicate the frequencies at which the second resonance is located for each simulation, and the vertical dotted line indicates the center frequency of the primary resonance. Note that the complex components and complex trajectories are mirror-symmetric, that the vibrational phase is always negative, and that the intensities of the two resonances sum together.

resonances are only linearly summing together rather than nonlinearly mixing.

The change in the spectra is dramatic when the weaker resonance is switched from the $\chi_{H}^{(3)}$ manifold to the $\chi_{L}^{(3)}$ manifold, as shown in Figs. 3.4 for identical parameters as those that generated Figs. 3.3. For a frequency spacing between the two resonances larger than a few natural linewidths there is little interference between them, and we see the characteristic sign inversion of the phase in line with the theory derived above. As the low- and high-frequency resonances approach and eventually pass through each other, the symmetry from Figs. 3.3 is replaced by a highly warped and asymmetric set of spectra. The most prominent example of interference is visible in the complex plane when the minor peak is set to $\Omega_{2}=20$ (cyan curves in Figs. 3.4). A two-fold amplification of the imaginary component of the resonance at $\omega \approx 0$ is observed. This particular feature of dual-frequency VPC-CARS can be used to enhance the response of a minor component in a sample, increasing the overall detection sensitivity so long as the majority and minority components are chosen to ensure that their 

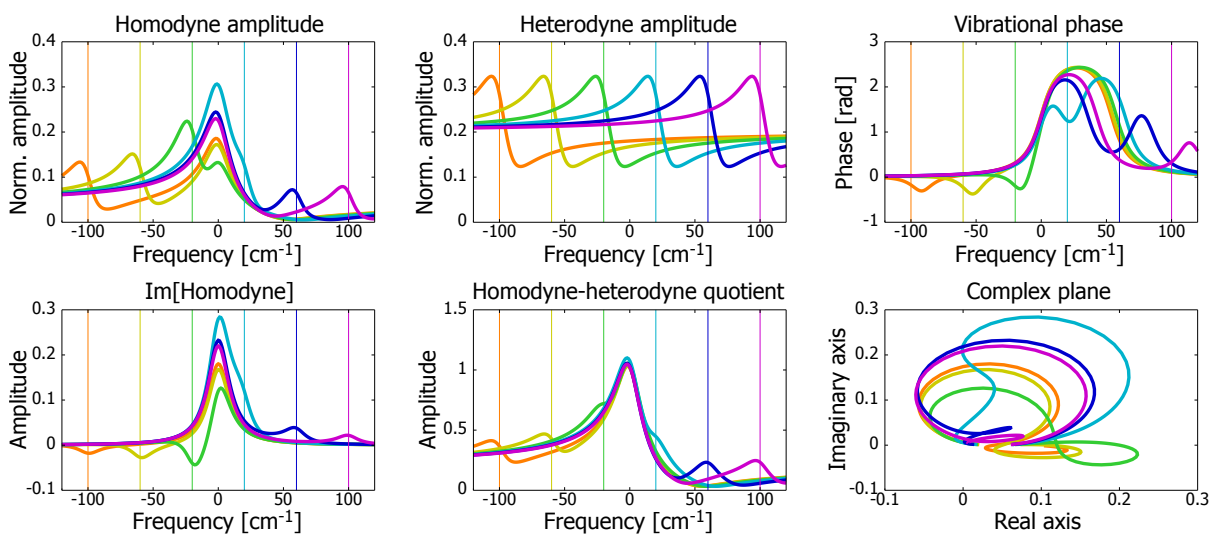

Figure 3.4. Two interfering resonances in different frequency manifolds. Plotted are (a) the intensity measured on the homodyne detector, (b) the amplitude measured on the heterodyne detector, (c) the vibrational phase, (d) the projection of the imaginary component of the homodyne intensity versus vibrational frequency, (e) the quotient of the local and heterodyne channels, and (f) the complex trajectory. Six curves are plotted, with a principal resonance located at $\Omega_{1}=0$ and a satellite minor resonance at values ranging from $\Omega_{2}=-100$ to $\Omega_{2}=+100$. The vertical colored lines indicate the frequencies at which the second resonance is located for each simulation, and the vertical dotted line indicates the center frequency of the primary resonance. Note the marked lack of mirror symmetry in the imaginary projection and the complex plane, which is a hallmark of frequency-dependent nonlinear interference.

resonances overlap appropriately.

Of particular note is that the quotient of the homodyne and heterodyne channels is identical for both measurement conditions. This can be proved mathematically by applying the parallelogram law to vectorial addition of polar and Cartesian representations of $\chi^{(3)}$ and working through a substantial amount of algebra. Because this quotient is unaffected by the number of vibrational manifolds that are simultaneously probed it provides an effective check when fitting experimental data to simulations.

\subsection{Experimental apparatus}

The optical setup used for detecting VPC-CARS is an expanded version of that described in the previous chapter. The most striking changes are the simultaneous use of three beams - the signal and the idler from the OPO, and the laser fundamental - and the introduction of frequency modulation. 


\subsubsection{Initial version}

The first implementation of a VPC-CARS experiment utilized an acousto-optic modulator (AOM) in the OPO signal beam to switch between homodyne and heterodyne detection schemes, as described by Jurna et al., which placed significant limits on image acquisition speed. Furthermore, this setup included a complicated phase-locked loop (PLL) to follow the laser repetition rate as it thermally drifted to deliver a constant $40-\mathrm{kHz}$ frequency modulation to the laser fundamental beam. This experimental setup could be adversely affected by three sources of noise. The first is the laser itself, which carries a spectrum of low-frequency fluctuations that is known as relative intensity noise (RIN). This RIN is informally called " $1 / \mathrm{f}$ " noise due to the inverse dependence of its amplitude on detection frequency, and it scales linearly with laser power. Second is shot noise, which is a statistical variation in the number of photons detected per unit time. Shot noise scales with proportionally to the square root of the detected laser intensity. Finally, the lumped circuit of the detector and its corresponding electronics form a constant background due to thermal effects and are independent of laser power.

Switching the OPO signal beam from high- to low-power mode for homodyne and heterodyne phase detection, respectively, was done in part because of the low modulation frequency. The heterodyne CARS detector was a small, low-noise silicon photodiode with a large transimpedance amplifier electrically behind it. At low local oscillator powers the detector noise was dominant, and heterodyne CARS signals cannot be recorded, while at high local oscillator powers the RIN is the dominant noise source. A range of local oscillator intensities between these two extreme zones is the shot-noise-limited region, wherein the detected heterodyne CARS signal scales faster than the associated shot-noise and therefore benefits from higher local oscillator powers. However, the real value of this local oscillator power is on the order of microwatts, not nearly enough to drive the homodyne CARS pathways. As a result the OPO signal was switched to low (microwatt) power during the heterodyne phase detection, and to high (tens of milliwatts) power for homodyne detection. This switching could only be accomplished at a few kilohertz due to a complicated set of electronics.

\subsubsection{Current version}

Two key breakthroughs in increasing the imaging rate of VPC-CARS were the introduction of higher modulation frequencies, and the removal of the OPO sig- 
nal power switching. The PLL has been removed in favor of directly driving the $\mathrm{AOM}$ at $85.7 \mathrm{MHz}$, which yields frequency sidebands on the laser fundamental beam at approximately $f_{r e p} \pm 5.8 \mathrm{MHz}$. Thermal drift of the laser repetition rate never exceeds a few $\mathrm{kHz} /$ hour, so the $5.8 \mathrm{MHz}$ frequency of the sidebands is effectively constant. Using a higher modulation frequency allows a higher imaging rate while still satisfying the Nyquist sampling criterion. Previously, with an $80-\mathrm{kHz}$ demodulation frequency, the maximum pixel update rate was about $30 \mathrm{kHz}$. With a demodulation frequency above $10 \mathrm{MHz}$, the Nyquist criterion allows acquisition rates of megapixels/second.

Using higher modulation frequencies also reduces the amplitude of the RIN to levels that are below the shot-noise limit even for local oscillator powers well into the hundreds of milliwatts. As a result it is no longer necessary to switch the local oscillator to low power for the heterodyne detection. Instead, we leave the OPO signal at constant high power, and detect the heterodyne channel with a large-area silicon photodiode. The large bias of $-60 \mathrm{~V}$ reduces the capacitance of the photodiode to allow it to effectively follow the high-frequency $(\approx 11.5 \mathrm{MHz})$ modulation, but is low enough that the photodiode cannot effectively follow the $(\approx 80 \mathrm{MHz})$ laser repetition rate. The laser therefore appears as a large DC offset. A simple passive RC high-pass filter consisting of a $100-\mathrm{nF}$ capacitor and a $50-\Omega$ resistor blocks this DC signal and transmits the modulated signal via a matched network to a lock-in amplifier. A full analysis of the noise in this setup is given in Appendix A.

The optical setup used in this chapter is shown in Fig. 3.5. The laser fundamental beam is directed through an AOM where it picks up 5.8-MHz frequency sidebands. It is then spatially and temporally overlapped with the signal and idler beams on dichroic mirrors after all three have been appropriately conditioned with telescopes and waveplates. The combined beams are colinearly directed into the inverved microscope. For transparent and/or weakly scattering samples the generated homodyne and heterodyne CARS fields are collected with an objective (0.55NA long working distance or 1.0NA water dipping) and directed to a short-pass dichroic mirror. The heterodyne CARS field is reflected toward a reverse-biased, 50- $\Omega$-terminated silicon photodiode while the homodyne CARS field is transmitted towards a red-sensitive PMT terminated with a large-bandwidth $30-\mathrm{k} \Omega$ transimpedance amplifier.

Highly-scattering or opaque samples preclude detection in transmission. The homodyne channel can be easily detected in a reflection geometry via a dichroic mirror. On the other hand the detected wavelength of the heterodyne channel is identical to one of the pump beams, so using a dichroic mirror is not possible. 


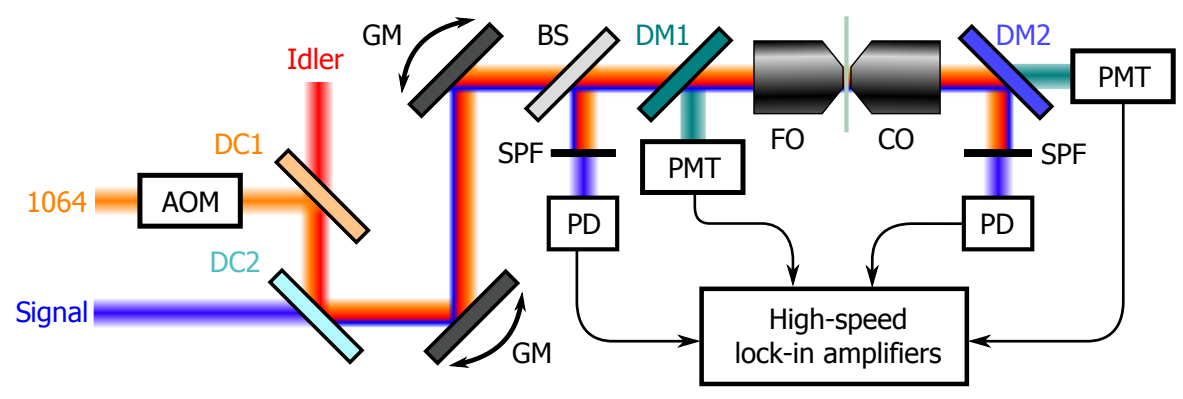

Figure 3.5. The optical setup used for VPC-CARS experiments. An acoustooptic modulator (AOM) applies a frequency modulation to the $1064 \mathrm{~nm}$ beam before it is combined with the signal and idler beams from the OPO. All three beams are colinearly launched into a laser-scanning microscopy via a pair of galvano mirrors (GM). A removable broadband beamsplitter (BS) is used for the detection of the reflected heterodyne CARS signal, while a long-pass dichroic mirror (DM1) splits the reflected homodyne CARS signal to a photomultipler tube (PMT). Transmitted CARS signals are directed to a short-pass dichroic mirror (DM2) at which the heterodyne CARS signal is reflected and the homodyne CARS signal is transmitted toward their respective detectors. All detectors feed their 50- $\Omega$-terminated outputs to high-frequency lock-in amplifiers.

Rather, a broadband beamsplitter must be installed. All three pump beams, covering nearly the entire optical octave between $800 \mathrm{~nm}$ and $1600 \mathrm{~nm}$, must be sent through this beamsplitter toward the sample, and the heterodyne CARS field and scattered local oscillator must then be reflected off of that same optic toward a detector. An optimization of the heterodyne intensity as a function of mirror reflectivity indicated that an optimum could be found at $23 \%$.

The outputs of the detectors are sent via electronic bandpass filters toward a set of high-speed lock-in amplifiers set to demodulate the second harmonic of the frequency modulation. The quadrature signals from the heterodyne and homodyne channels are sent to an external data acquisition card (DAQ) read by custom LabVIEW software. This software calculates the heterodyne amplitude and vibrational phase on a pixel-by-pixel basis in real time. Acquisition speeds up to 200,000 pixels per second $(200 \mathrm{kpx} / \mathrm{s})$ are possible, with lock-in time constants as low as $780 \mathrm{~ns}$. 


\subsection{Results}

\subsubsection{Epi-detection}

In a tightly-focused excitation geometry there is a strong relationship between the size of the resonant object and the detected CARS intensity[121]. Large objects scatter much more efficiently into the direction of illumination, while objects on the order of the wavelength of the anti-Stokes wavelength radiate nearly isotropically. However, the phase of the radiated anti-Stokes field is independent of the physical direction of the scattered light. Experimentally it is challenging to conclusively demonstrate the difference between epi-generated CARS signals and scattered forward-generated signals from small objects; timecorrelated detection does not have the temporal resolution to distinguish between the two pathways, and the collinear excitation geometry we use does not provide any suitable spatial parameters to tweak. A good option to minimize scattering while allowing epi-generation is to use small resonant objects immersed in index-matching fluid. In our case we choose tristearin, the same triglyceride used in the previous chapter, submerged in paraffin, a liquid longchain alkane. The indices of refraction of these two compounds are similar. We selected a small $(<1 \mu \mathrm{m})$ piece of tristearin as our target and recorded VPC-CARS spectra simultaneously in both transmission and reflection. The amplitudes, vibrational phases, and complex trajectories for the two detection geometries are shown overlaid in Figs. 3.6. Good agreement is seen over most of the vibrational spectrum.

Pharmaceutical tablets, such as the one displayed in Fig. 2.12 in the previous chapter, are not compatible with transmission measurements. They are thick and scatter light efficiently, which provides an ideal medium for epi-detection. We apply VPC-CARS microscopy to a tablet containing 50\% tristearin, $45 \%$ diprophylline, and $5 \% \beta$-mannitol. Noting from the previous chapter that all of these compounds can exist in multiple crystal forms and/or orientations we proceed with caution, and acquire images at $2850 \mathrm{~cm}^{-1}$ and $2955 \mathrm{~cm}^{-1}$. These frequencies are selected because they offer maximum specificity between the three compounds regardless of their orientations and crystal forms. The resulting (single-frequency) images are shown in Figs. 3.7.

Interpretation of the frames containing amplitude and phase information is straightforward; the high-intensity tristearin resonance at $2850 \mathrm{~cm}^{-1}$ is easily visible against the dark mannitol and diprophylline in Fig. 3.7(a), and its corresponding phase further differentiates it from the crystals (Fig. 3.7(b)). On the other hand the mannitol and diprophylline resonances, indistinguishable at 

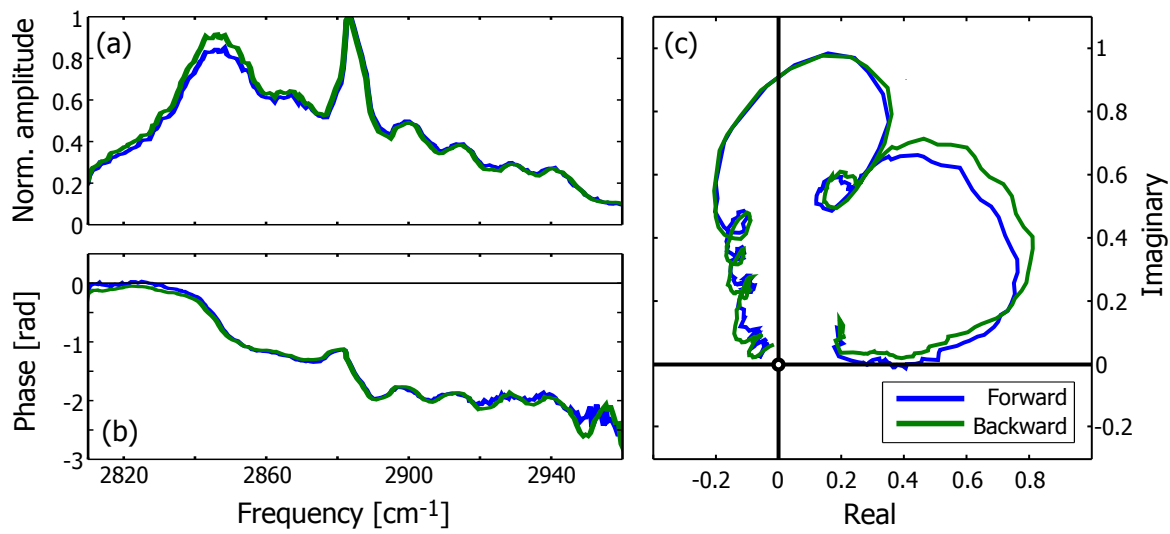

Figure 3.6. Transmitted (blue) and epi-detected (green) VPC-CARS spectra of tristearin in paraffin liquid. The normalized amplitudes (a), phases (b), and complex trajectories (c) generally agree well between the two detection geometries. A small amplitude deviation around $2850 \mathrm{~cm}^{-1}$ is attributed to a small extra contribution from the paraffin, which does not contain the strong resonance near $2880 \mathrm{~cm}^{-1}$ that tristearin displays.

$2850 \mathrm{~cm}^{-1}$, become distinct when measured at $2955 \mathrm{~cm}^{-1}$. The diprophylline has a slightly larger vibrational phase than the mannitol (Fig. 3.7(e)), but similar intensity (Fig. 3.7(d)). When plotted as density histograms in the complex plane, distinct regions emerge corresponding to the locations and relative abundances of each compounds. At $2850 \mathrm{~cm}^{-1}$ the tristearin is well separated from the mannitol and diprophylline due to being resonantly driven. The mannitol and diprophylline are nearly indistinguishable. However, at $2955 \mathrm{~cm}^{-1}$ all three compounds are well separated in the complex plane, and can be readily distinguished even though their amplitudes are similar. The fringes that appear in the phase images are due to the presence of the broadband beamsplitter, which introduces phase delays between the heterodyne anti-Stokes field and the local oscillator that cannot be compensated by detecting the homodyne phase. Spatial filtering via a Fourier transform is used to remove most of the fringe amplitude, but as the oscillation is not truly periodic there is still a minor residual error after the filtering.

\subsubsection{Spectral unmixing of complex data}

Hyperspectral VPC-CARS data presents a unique analysis problem. The data is inherent five-dimensional, containing two spatial coordinates, a frequency 


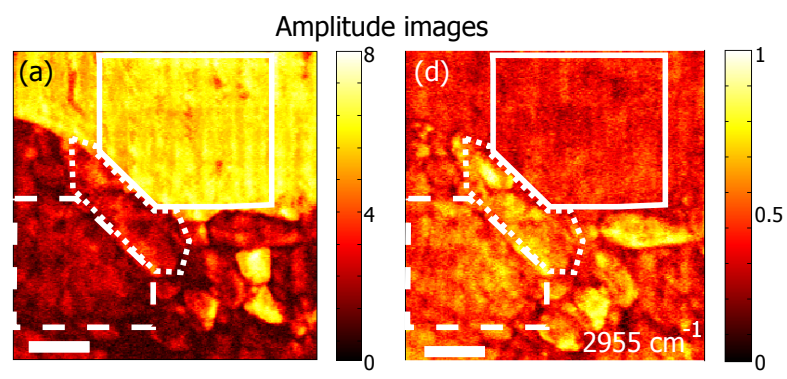

Vibrational phase images
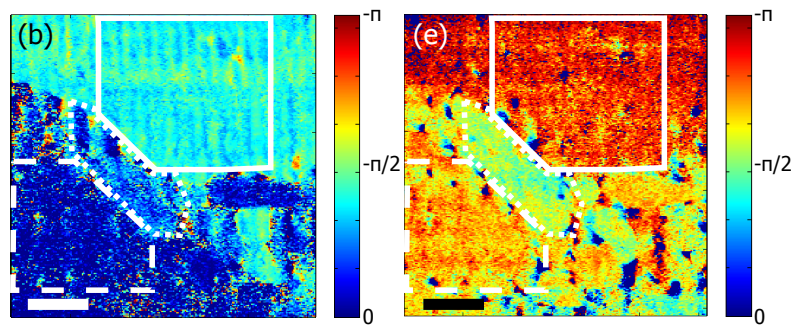

Complex plane plots

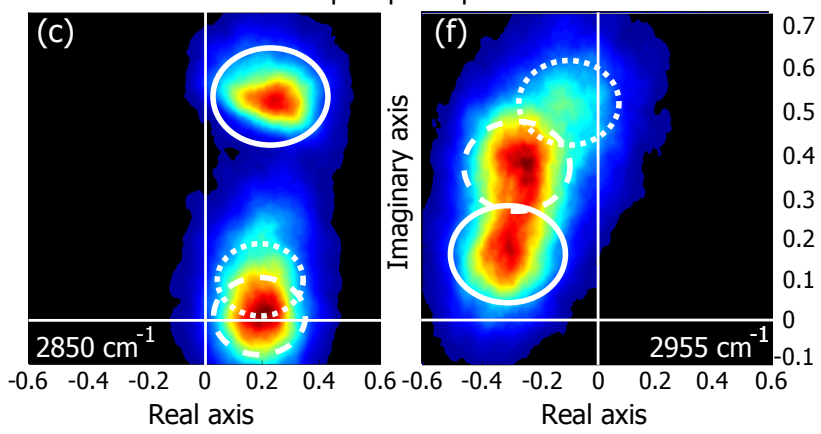

Figure 3.7. Images of a pharmaceutical tablet recorded with epi-detected VPC-CARS at $2850 \mathrm{~cm}^{-1}$ (left column) and $2955 \mathrm{~cm}^{-1}$ (right column). The amplitude images (top row), phase images (middle row), and complex plane density plots (bottom row) show distinct differences between the indicated regions at the two frequencies. The solid border is tristearin, the long-dashed border is mannitol, and the short-dotted border is diprophylline.

coordinate, and both axes of the complex plane. A data reduction system such as that used for hyperspectral CARS in the previous chapter can only be applied to four of these dimensions. While it is possible to extract the imaginary (resonant) component and perform the projections on it, there is more value to be gained by analyzing the entire complex plane. We have performed two types 
of analysis on hyperspectral complex vibrational data. First, we use simple overlap integrals on individual pairs of pixels to build clusters with similar spectra. The spatial extent of these clusters indicate where certain spectral features are located within the image. This method is very quick and dirty insofar as it is not possible to extract the underlying raw spectral components. When there is minimal sub-diffraction mixing within a sample this method is sufficient to determine quantitative ratios of compounds within a sample, provided that the spectra of the pure materials are known a priori. Samples that are mixed at the sub-micron level require more sophisticated spectral unmixing. To that end we turn to a spectral decomposition algorithm first developed by Alina Zare and Paul Gader[22, 122] called Sparsity Promoting Iterative Constrained Endmember detection, or SPICE for short.

\section{Overlap integral clustering}

A complex hyperspectral data set can be depicted as a set of complex numbers in three-dimensional spatiospectral space $\tilde{S}(x, y, \Delta \omega)$ where $x$ and $y$ are spatial locations within the image and $\Delta \omega$ is the driving difference frequency. The data from high-frequency VPC-CARS in the absence of electronic resonances is expected to sit entirely in the upper half of the complex plane, so that $\Im[\tilde{S}(x, y, \Delta \omega)]>0$. Due to the field-of-view curvature of the objective the sample will be non-uniformly illuminated, and chromatic aberrations are expected for low magnifications. It is therefore imperative that a normalization take place that does not affect the relative spectral relationships between different spatial locations within the image. We begin by first subtracting the individual means of the real and imaginary components so that

$$
\int \tilde{S}_{n o r m}(x, y, \Delta \omega) d(\Delta \omega)=0
$$

which centers all of the spectra in the whole image at the origin of the complex plane. Second, we determine a normalization constant $r$ for each spatial pixel such that

$$
\frac{1}{r(x, y)} \int \tilde{S}_{n o r m}(x, y, \Delta \omega) \tilde{S}_{n o r m}^{*}(x, y, \Delta \omega) d(\Delta \omega)=1 .
$$

These two operations yield a mean-normalized, unity-variance data set, in which the spectra of any two pixels can be directly compared by calculating 
their overlap integral

$$
\nu=\int \tilde{S}_{n o r m}\left(x_{1}, y_{1}, \Delta \omega\right) \tilde{S}_{n o r m}^{*}\left(x_{2}, y_{2}, \Delta \omega\right) d(\Delta \omega) \subset[0,1]
$$

where a value of zero indicates total dissimilarity and unity indicates perfect similarity. Pixels with very high values of the overlap integral are considered to be composed of a similar substance. The threshold value is arbitrarily defined; for data sets with very low noise, the threshold can be set to $\nu=0.90$ or higher, while high-noise data sets necessarily require a relaxation of the threshold criterion.

The overlap integral computation is computationally intensive, the time to complete the operation scales with the square of the linear image dimension, and the data produced is still half the size of the original data set. These features mean that one-to-one comparison of all pixels in the image is unfeasible for any image of reasonable size. Instead we use a sparse subset of pixels as references by which to compare all other pixels in the image. We select a single pixel at random and compare its spectrum to all others in the image. All pixels that satisfy the overlap integral threshold condition are then binned together and removed from the unclustered set. A new pixel from this unclustered set is selected as the next reference, and the process is repeated until mostnormally more than $90 \%$ - of the pixels in the image are clustered. Maps of the distributions of the pixels as well as their reference spectra are then plotted.

We apply this clustering method on a complicated sample of quench-cooled mannitol. This data set consisting of $512 \times 512 \times 65$ complex voxels, acquired at a rate of $10 \mathrm{kpx} / \mathrm{s}$ with a $25 \mu \mathrm{s}$ time constant, covers $120 \mu \mathrm{m}$ and $180 \mathrm{~cm}^{-1}$. A map of the clusters in Fig. 3.8(b) shows very defined zones around an apparent nucleation site; the imaginary projections of the complex spectra (Fig. 3.8(a)) from the regions surrounding the nucleation site agree with the $\delta$-mannitol spectra in Fig. 2.11. Perfect agreement is not expected; the data shown in the previous chapter is from a modulus-square CARS measurement that includes mixing of the resonant and non-resonant components. Six separate colors are assigned to regions with approximate $\delta$-mannitol spectra, indicating either differences in orientation or mixtures with small amounts of a different crystal form. Also present in the image are no fewer than eight different spectra associated with $\alpha$-mannitol. The normalization process removes the absolute differences in peak heights that characterize the orientational Raman spectra of this polymorph, but relative variations are still readily apparent; components three and four, as examples, are two nearly orthogonal orientations of 


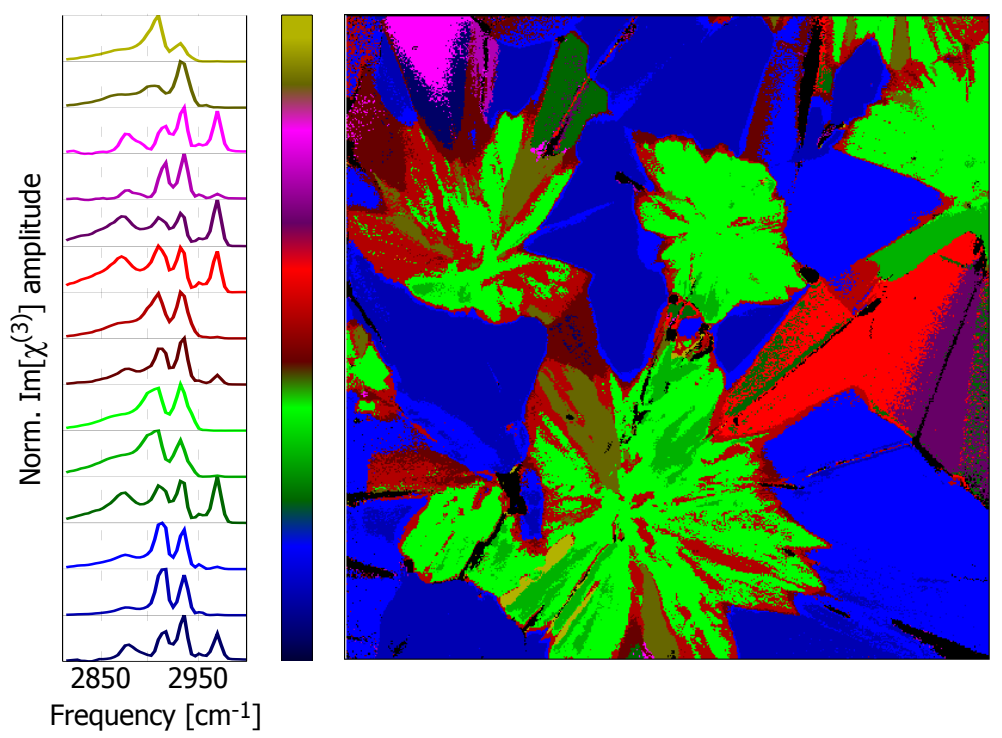

Figure 3.8. Spectral overlap integral clustering of normalized complex VPCCARS data acquired at $10 \mathrm{kpx} / \mathrm{s}$ over $180 \mathrm{~cm}^{-1}$. The image is $120 \mu \mathrm{m} \times 120 \mu \mathrm{m}$ with a clustering overlap threshold of $\nu=0.95$. The left column (a) shows the imaginary components of the spectra in each region of the sample, color-coded so that the spectral lines match the image cluster color (b).

pure $\alpha$-mannitol. The spectral overlap integral between these two components is $\nu \approx 0.5$, demonstrating that although this method is useful for identifying differences between two regions it cannot be used to determine the underlying basis spectra. By this clustering analysis we estimate that there are five or six distinct components present in this sample.

\section{SPICE}

The field of (hyper)spectral unmixing is broad and diverse, with a plethora of options available to suit nearly any requirement. With so many techniques on the market the motivation for choosing SPICE over more established methods like principal component analysis (PCA) is important. Component analysis routines merely attempt to minimize the number of orthogonal spectral components that describe the full data set without regard to reproducing the underlying real data. Worse still, PCA requires that all components must be mean-normalized to zero, which yields negative-valued spectral points even when the underlying data is positive. The results from this class of algorithms 
therefore require very careful analysis. The final nail in the coffin of PCA is that it ranks components in terms of their mutual variance from all other components. Under-represented components of the image can therefore be buried in a low-ranking component even when their spectra vary substantially from all other components in the image. This combination of negative traits makes PCA an unfavorable candidate for spectral unmixing.

On the other hand endmember detection and identification algorithms are concerned with extracting the pure spectra of mixed data sets, and the results correspond directly to physical spectra. Endmember identification routines generally attempt to minimize a residual sum of squares (RSS). The SPICE algorithm first takes every pixel in the scene to have a spectrum

$$
\mathbf{S}_{i}=\sum_{k=1}^{M} p_{i k} \mathbf{E}_{k}+\varepsilon_{i} i=1, \ldots, N
$$

where $N$ is the number of pixels in the image, $M$ is the number of endmembers, $\mathbf{E}_{k}$ is the spectrum of the $k^{t h}$ endmember, and $p_{i k}$ is the proportion of endmember $k$ in pixel $i$. The proportionality constants must satisfy

$$
\sum_{k=1}^{M} p_{i k}=1, p_{i k} \geq 0, k=1, \ldots, M .
$$

Minimization then proceeds according to a convex geometry model akin to a rubber band being enclosed around a number of nails in a board: minimizing the RSS is accomplished by a combination of iterative fitting and selective pruning of unnecessary endmember candidates. A visual depiction of the process is given in Fig. 3.9. The mathematics behind the SPICE algorithm is found described in detail in Ref. [123], with additional endmember selection procedures published in Ref. [122].

Since it was originally developed for analyzing hyperspectral satellite imagery, SPICE was historically only used with real, positive data. However, there are no internal constraints that limit it to either real or positive spectral values, which is a critical consideration for analysis of VPC-CARS data. Further, there is no mean normalization or variance scaling in SPICE, which allows quantitative descriptions of endmember mixing on a per-pixel basis.

There are two variable parameters, $\mu$ and $\Gamma$, that can control the convergence behavior of the SPICE algorithm. The $\mu$ parameter defines the volume enclosed by the estimated endmembers; larger values of $\mu$ are useful when there is substantial noise in the signal, or when there is minimal mixing of endmem- 

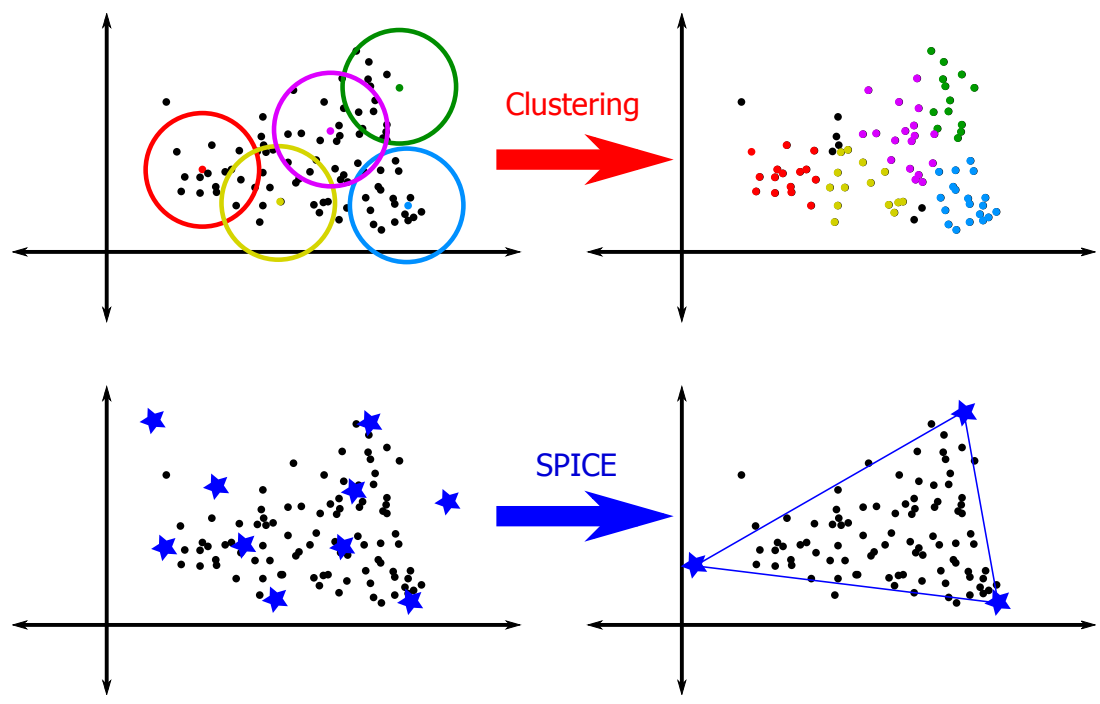

Figure 3.9. Conceptual principles of the blind clustering and SPICE methods. A set of data is processed so that it can be represented in 2D space, forming the distribution of black dots. (Top) Random points are selected sequentially from the data set as prototypes. Nearby data points, i.e. those with similar spectra, are clustered together with the prototypes. The distance threshold and number of unclustered pixels are independent parameters. (Bottom) SPICE takes a random distribution of endmember candidates and moves them around the data set until a specific convergence criterion is satisfied. Data points outside of the endmember convex hull are accounted for with an error term. SPICE attempts to determine the best endmember set for given data, and can be tailored to specific situations with two independent parameters.

bers throughout the image. Tuning $\mu$ enables the algorithm to find the tightest endmember fit around the data. The aggressiveness of the algorithm to prune endmembers is influenced by the value of $\Gamma$. Smaller values of $\Gamma$ generally yield more endmembers as SPICE is less prone to reject candidates, while larger values will yield smaller endmember sets.

Using SPICE to identify endmembers in a complex VPC-CARS data set is untested and the ideal $\mu$ and $\Gamma$ parameters are unknown. As part of a collaborative effort Dr. Alina Zare has modified the SPICE code to run on the full complex data set obtained with VPC-CARS. We use the same mean-normalized, unity-variance data described in the previous section to test these parameters. Every permutation of $\mu=[0.01,0.05,0.1]$ and $\Gamma=[10,50,100,500]$ are applied to three analysis runs each for a total of 36 sets of estimated endmembers and 


\begin{tabular}{c|cccc}
\hline & $\Gamma=10$ & $\Gamma=50$ & $\Gamma=100$ & $\Gamma=500$ \\
\hline$\mu=0.01$ & {$[18,15,17]$} & {$[18,14,13]$} & {$[9,9,10]$} & {$[5,6,6]$} \\
$\mu=0.05$ & {$[11,14,12]$} & {$[8,11,9]$} & {$[9,9,6]$} & {$[6,4,5]$} \\
$\mu=0.1$ & {$[12,10,10]$} & {$[10,7,7]$} & {$[5,6,7]$} & {$[4,4,4]$} \\
\hline
\end{tabular}

Table 3.1. Number of estimated endmembers for each combination of $\mu$ and $\Gamma$ in three separate runs. The number of estimated endmembers from the clustering analysis is five or six.

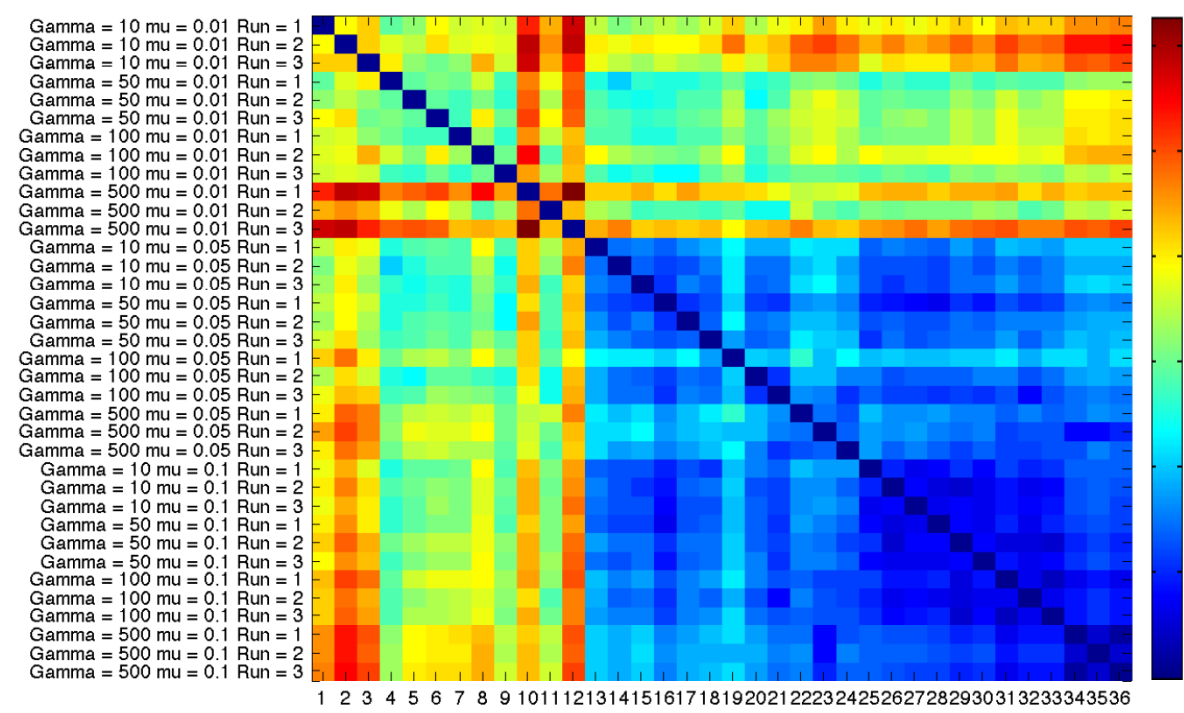

Figure 3.10. Pair-wise Earth Mover's Distance results for the 36 runs of the SPICE algorithm on the VPC-CARS data of quench-cooled mannitol. The centroid result is at $\Gamma=50, \mu=0.05$, Run 1 .

proportion constants. The number of endmembers estimated in these runs for each pair of the $(\mu, \Gamma)$ parameters are shown in Table 3.1.

Comparison with the clustering results indicates that most parameter sets overestimate the number of endmembers in the sample. To provide a more quantitative measure of the ability of SPICE to estimate the endmembers of the complex VPC-CARS data set we turn to the Earth Mover's Distance (EMD) algorithm[124, 125]. The EMD computes the least work required to transform one data element into a different data element within a single parameter space. A physical analog is to envision two different piles of sand on identical plots of land. An EMD analysis will provide the least work required to transform 

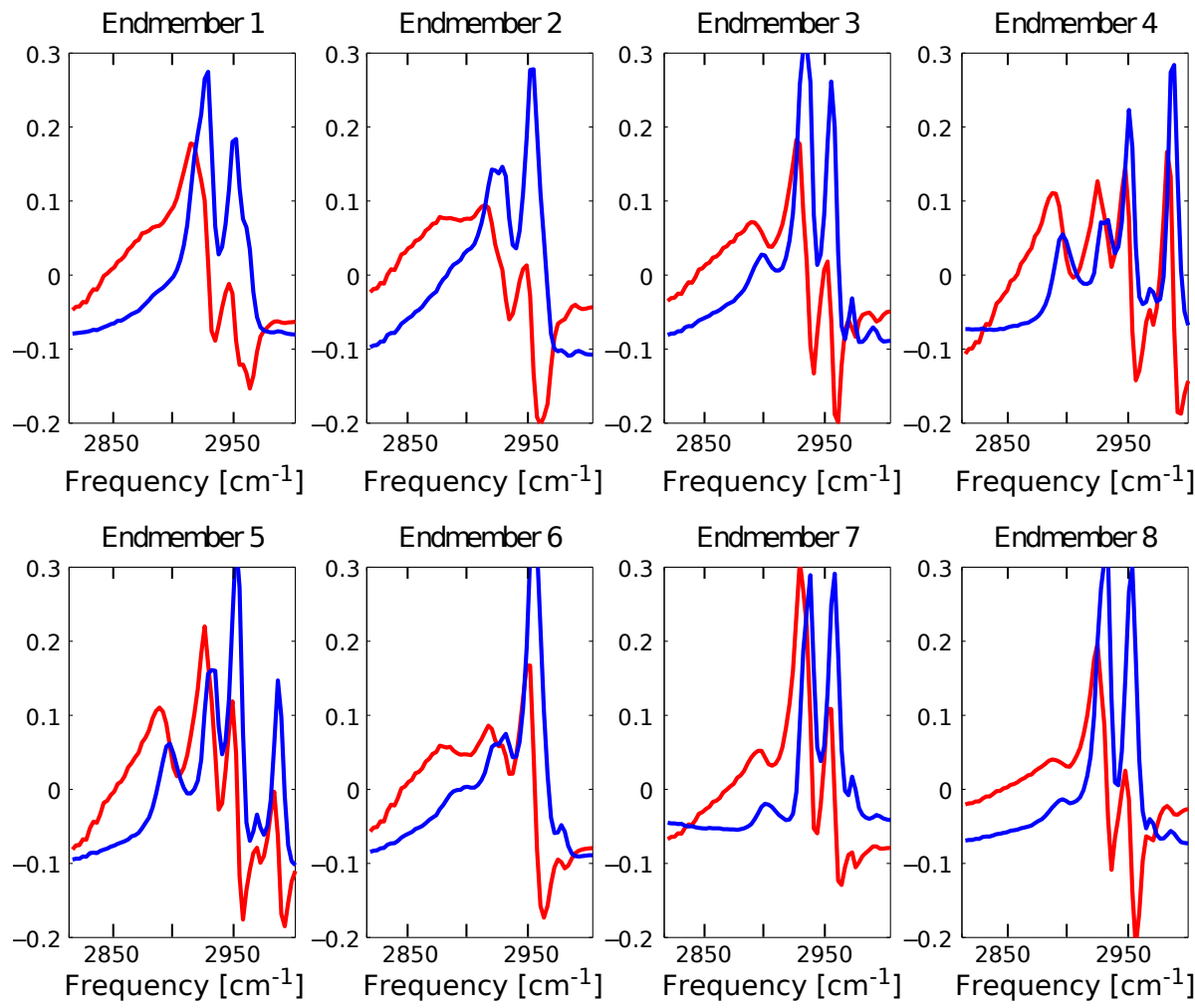

Figure 3.11. Endmembers of the centroid result from the Earth Mover's Distance analysis. The real (red) and imaginary (blue) components of each endmember are plotted here. Orientations of the $\delta$-mannitol polymorph are observed in endmembers 1,2 , and 6 , while $\alpha$-mannitol represents the rest. Mixing of the $\alpha$ and $\delta$ forms might be responsible for the excessive number of $\alpha$-mannitol endmembers. Note that SPICE operated on the mean-normalized, unity-variance data; in reality the imaginary values should be positive, and the real values should have positive off-resonance values at the edges of the spectrum corresponding to the magnitude of the non-resonant background.

one pile of sand into the other on an optimized grain-by-grain basis. Using the EMD with all of the pair-wise SPICE results will yield an estimation of how closely the results agree with each other for different parameter values. All pair-wise EMD values are shown in Fig. 3.10. The representative SPICE endmember run is the one with the lowest summed pair-wise EMD value across all other SPICE runs. This "centroid" result is $\Gamma=50, \mu=0.05$, Run 1.

The centroid result, as seen from Table 3.1, contains eight endmembers. The 


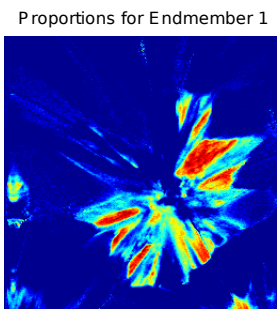

Proportions for Endmember 5

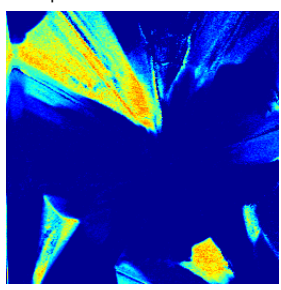

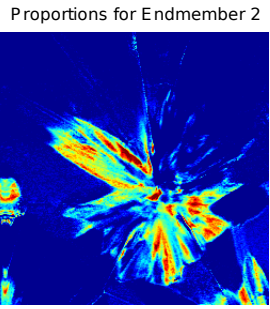

Proportions for Endmember 6

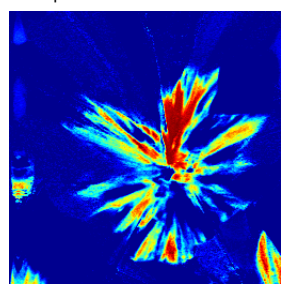

Proportions for Endmember 3

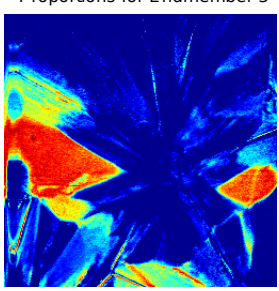

Proportions for Endmember 7

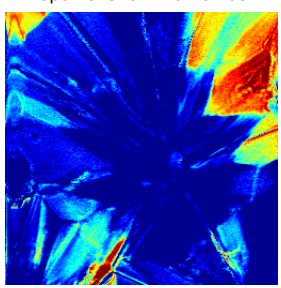

Proportions for Endmember 4

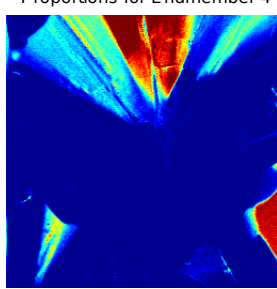

Proportions for Endmember 8

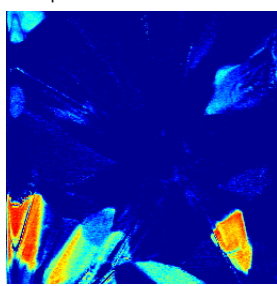

Figure 3.12. Proportion values of each endmember from the centroid result of the Earth Mover's Distance analysis. The material surrounding an apparent nucleation site in the center of the image all corresponds to the $\delta$-mannitol polymorph, in line with expectations, while the $\alpha$-mannitol form is seen further away from the nucleation site. No $\beta$-mannitol is observed.

spectrum of each pixel in the image, as defined in Eq. 3.18 according to the conditions in Eq. 3.19, is the sum over all endmembers scaled by a proportion factor. The complex Cartesian components of the endmembers of the centroid result are plotted in Figs. 3.11, and the spatial distributions of the proportions of each endmember are shown in Figs. 3.12. The general distribution of the $\alpha$ and $\delta$-mannitol forms agrees with expectations and previous observations on quench-cooled samples; the reproducibility of this result is very high. Unfortunately the SPICE algorithm over-estimates the number of spectra associated with $\alpha$-mannitol. The likely reason is that five distinct non-orthogonal orientations of $\alpha$-mannitol are present within this one image, and SPICE is not constrained heavily enough to tightly fit the available spectral data.

The effect of tightening the parameters - increasing $\Gamma$ to 100 and $\mu$ to 0.1 - is seen in Figs. 3.13 and 3.14. This set of parameters results in a reduction to six endmembers, in line with estimates from the clustering analysis. However, while two of these endmembers show very high specificity for the $\delta$ polymorph, four of the endmembers are still associated with the $\alpha$ form. Comparing the imaginary components of the endmembers to the spontaneous Raman spectra of Beattie et al.[105], we find that endmembers 1-3 agree with the three orthogonal 
Endmember 1

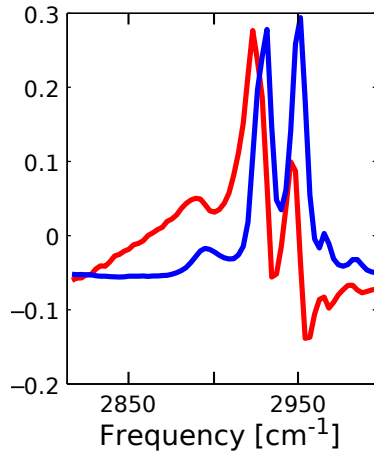

Endmember 4

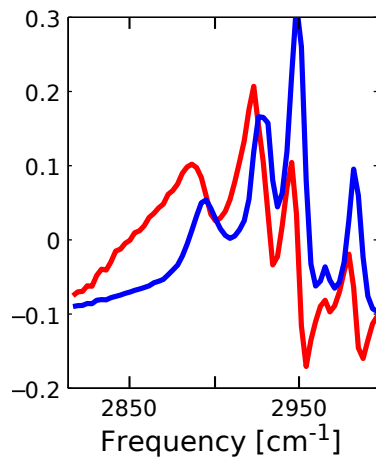

Endmember 2

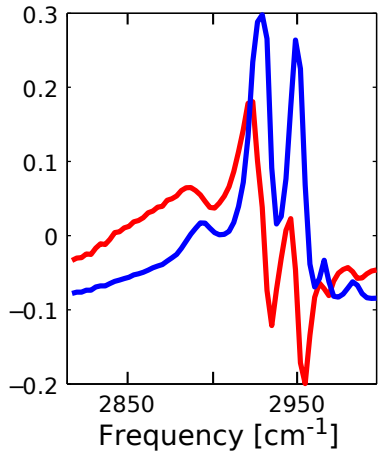

Endmember 5

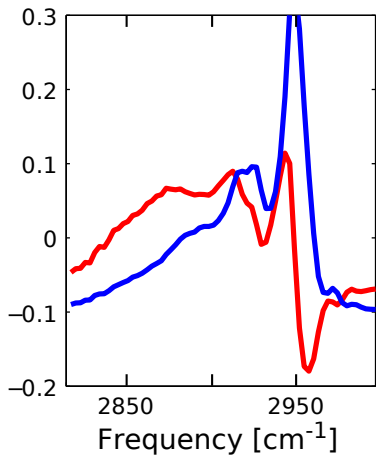

Endmember 3

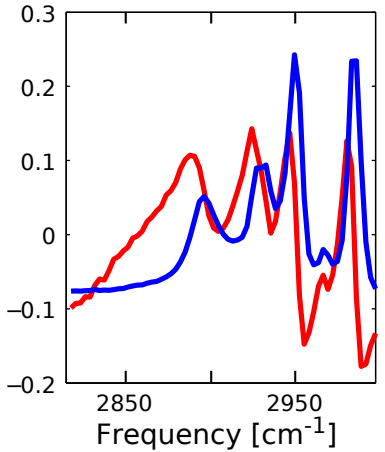

Endmember 6

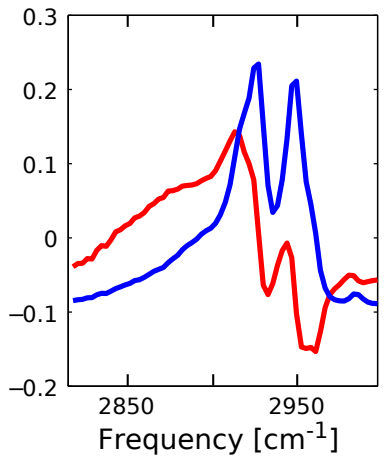

Figure 3.13. Endmembers of a run with $\Gamma=100, \mu=0.1$. The real (red) and imaginary (blue) components of each endmember are plotted here. Orientations of the $\delta$-mannitol polymorph are observed in endmembers 5 and 6 , while $\alpha$-mannitol represents the rest. While the $\delta$ form is clearly isolated by SPICE, the $\alpha$ endmembers still have not fully converged onto the three orientational basis spectra. Note that SPICE operated on the mean-normalized, unity-variance data; in reality the imaginary values should be positive, and the real values should have positive off-resonance values at the edges of the spectrum corresponding to the magnitude of the non-resonant background.

orientations. Endmember 4 appears to be a combination of endmembers 1 and 3 ; with more stringent fitting parameters it may disappear entirely.

All of the data presented in this section was recorded at the relatively quick acquisition rate of $50 \mathrm{kpx} / \mathrm{s}$. We justify this high acquisition rate because mannitol is a very easy sample to work with: it has a high damage threshold, strong resonances, is effectively transparent with our sample preparation, and does not rapidly degrade in ambient conditions. As a first step toward further 
Proportions for Endmember 1

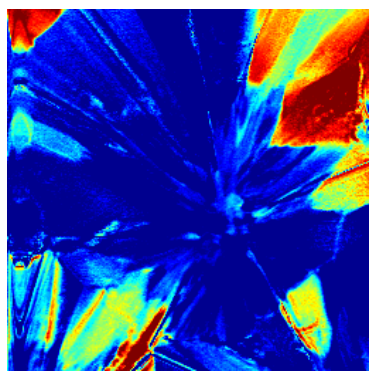

Proportions for Endmember 4

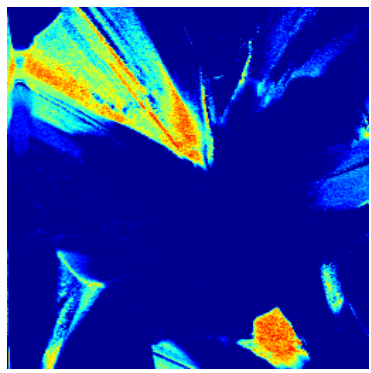

Proportions for Endmember 2

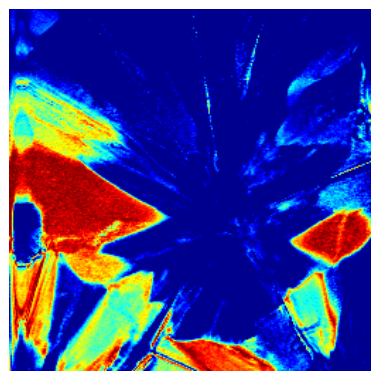

Proportions for Endmember 5

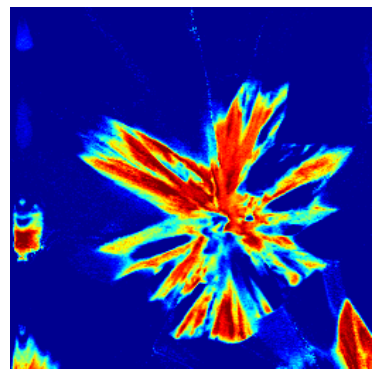

Proportions for Endmember 3

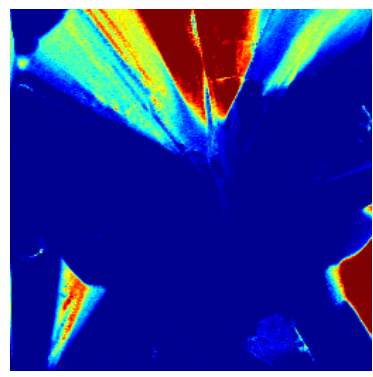

Proportions for Endmember 6

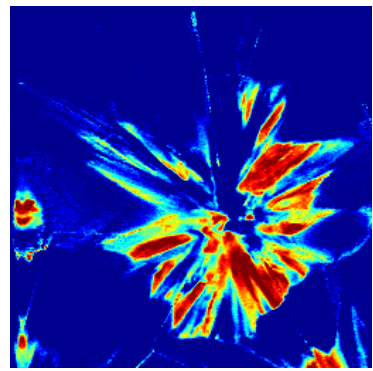

Figure 3.14. Proportion values of each endmember with $\Gamma=100$ and $\mu=0.1$. The material surrounding an apparent nucleation site in the center of the image all corresponds to the $\delta$-mannitol polymorph, in line with expectations, while the $\alpha$-mannitol form is seen further away from the nucleation site. No $\beta$-mannitol is observed.

applications involving hyperspectral VPC-CARS these results are promising. For example, experiments on tissue necessitate much lower optical powers and yield weaker signals. Longer integration times are therefore required to obtain comparable a signal-to-noise ratio as we have demonstrated. However, at this speed the data is not shot-noise-limited, and electronic noise is expected to play a large role in degrading the signal quality. With smaller acquisition bandwidths and appropriate electronics the detection could become shot-noiselimited. In both types of applications, SPICE (and all other endmember algorithms) must fulfill a tall order of determining endmembers through noise and other complicating factors. With the results presented above we believe that we have justified the use of SPICE and EMD for analyzing the complex hyperspectral data that we obtain with VPC-CARS. 


\subsubsection{High-speed phase-sensitive imaging}

With the current implementation of the VPC-CARS setup acquisition rates up to $200 \mathrm{kpx} / \mathrm{s}$ are theoretically possible. In practice the limit for reliable imaging is about $50 \mathrm{kpx} / \mathrm{s}$. Beyond this speed it is challenging to synchronize the commercial microscope control software with custom LabVIEW analysis software. Both programs require a stable pixel clock, as well as digital signals indicating the beginning and end of each line and each frame. The microscope control software can be driven with either an internal pixel clock that is inaccessible to external devices, or with an external TTL-level pixel clock source. The former leads to unpredictable jitter, so time-lapse and hyperspectral imaging are not feasible. We therefore use an external clock.

For the option of both systems running off of the same external clock the problem lies in how the control software internally processes an external clock signal. While we do not fully understand the internal workings - the control software is proprietary and the source code is not available-high pixel clock rates result in an offset between the TTL Line Active signal and the acquisition of the first pixel of that line. The offset appears to be proportional to the pixel clock rate, and is non-trivial for clock rates above $20 \mathrm{kpx} / \mathrm{s}$. Images containing this offset at different pixel rates are shown in Figs. 3.15. Note that the "extra" pixels on the left side of the image contain real data from the return stroke of the galvano mirrors. While the synchronization between the TTL Line Active signal and image acquisition is poor, the timing jitter between the control and LabVIEW software is minimal in this configuration. This synchronization method is therefore valid for hyperspectral and time-lapse imaging.

\subsubsection{Dual-frequency VPC-CARS}

Analysis of VPC-CARS in the fingerprint region is significantly complicated by the presence of highly resonant alkyl modes in the $3000 \mathrm{~cm}^{-1}$ region. Acquisition of the data is not significantly more challenging than for high-frequency VPC-CARS. The difficulty in analysis comes from fitting resonance parameters to the resulting data. Because of the strong optical coupling between the lowand high-frequency vibrational modes, both the resonant Lorentzian terms and the absolute magnitude of the non-resonant background are critical.

We choose DMSO for a prototypical sample to demonstrate these coupling effects and the difficulty in fitting the model to experimental data. The vibrational spectrum of DMSO is uncomplicated, containing only a few $\mathrm{CH}$ modes 


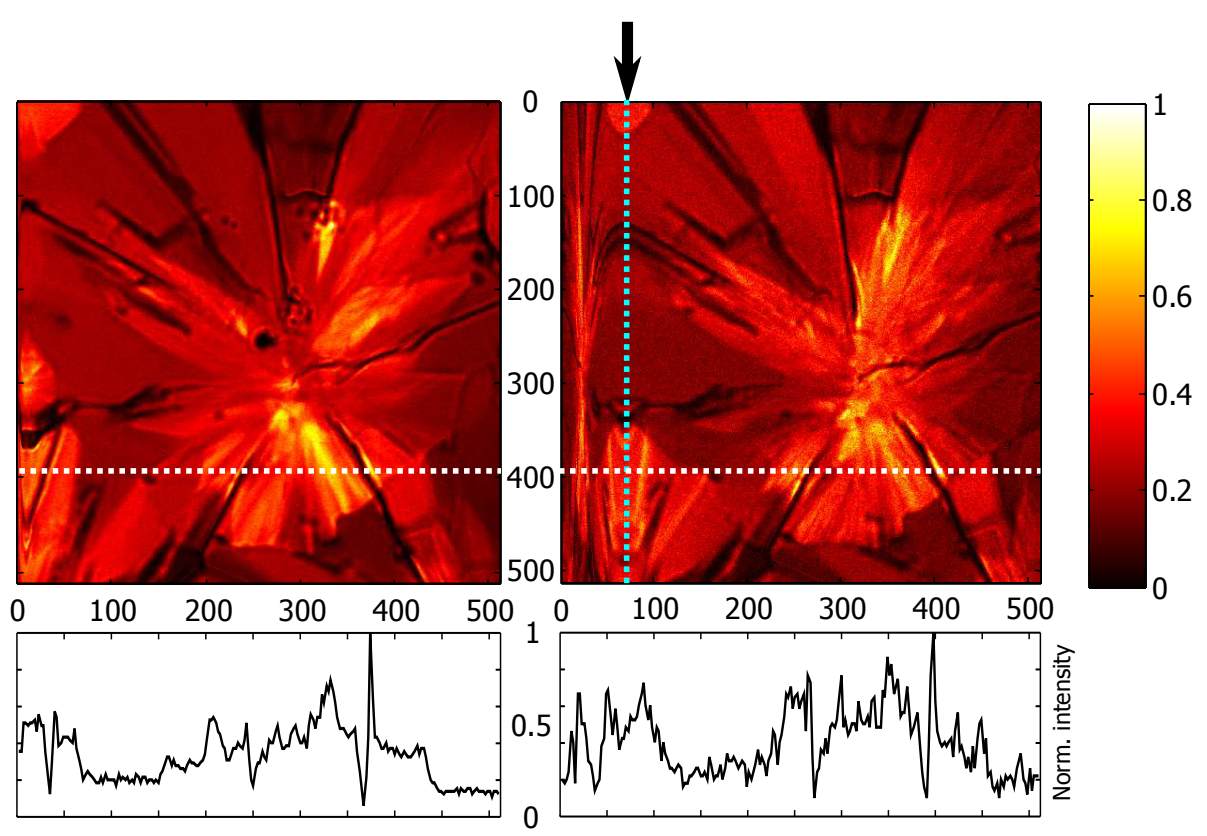

Figure 3.15. Comparison of (left) low- and (right) high-speed VPC-CARS microscopy, at acquisition rates of $10 \mathrm{kpx} / \mathrm{s}$ and $200 \mathrm{kpx} / \mathrm{s}$, respectively. The two images show the CARS amplitudes of the same region of a quench-cooled mannitol sample, both taken with an external pixel clock at $2890 \mathrm{~cm}^{-1}$. Horizontal and vertical scales are in pixels. The image is $120 \mu \mathrm{m} \times 120 \mu \mathrm{m}$. Note the compressed mirroring of the data on the left side of the high-speed image, indicate by an arrow. Small differences between the two images are a result of focus drift; these images are individual frames within hyperspectral stacks. The intensity cross-section at the indicated line is shown below the images. The deviation between spatial features in the two images is greater on the left side than on the right, indicating that the galvano mirrors begin moving non-linearly at high external pixel clock rates.

and a relatively empty fingerprint. The most prominent peaks in these two regions are listed below in Table 3.2, reproduced from Martens et al[126]. We use these empirical values as a starting point to fit our model to the VPC-CARS data. Unfortunately, using these exact values with a reasonable non-resonant background ( $2 \%$ of maximum resonance amplitude) generates a spectrum that does not agree with data at all (Figs. 3.16). Using a manual method we find a set of amplitude and linewidths for each vibrational frequency that fits quite closely with the data (Figs. 3.17). There are six individual plots in each Figure. 

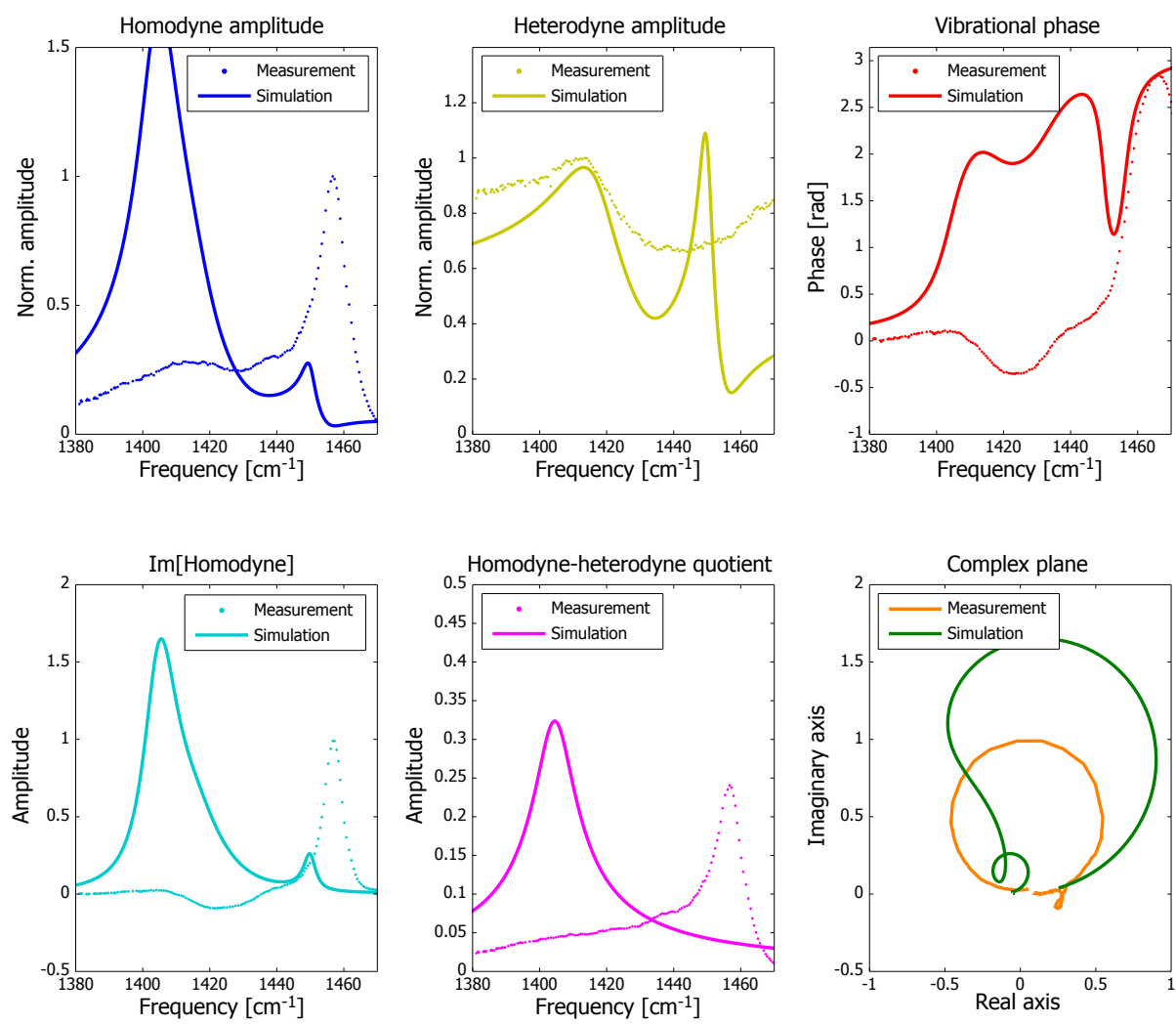

Figure 3.16. Measurements and simulations of a dual-frequency VPC-CARS spectrum of neat DMSO in the fingerprint region using published vibrational mode parameters. Agreement between theory and experiment is poor.

They correspond to: the modulated intensity detected on the homodyne channel, which is equivalent to Eq. 3.11; the heterodyne amplitude from Eq. 3.13; the vibrational phase, which is the difference of the phases detected on the heterodyne and homodyne channels; the imaginary component of the homodyne channel calculated as the product of the homodyne channel amplitude and the sine of the vibrational phase; the quotient of the homodyne intensity and the heterodyne amplitude; and the complex representation of the homodyne amplitude and phase.

Good agreement between the model and experimental results is not unexpected, but it is interesting that high-resolution spontaneous Raman spectroscopy data does not provide valid amplitude and linewidth parameters for the model. Further evidence of strange behavior is seen in multiple places across 

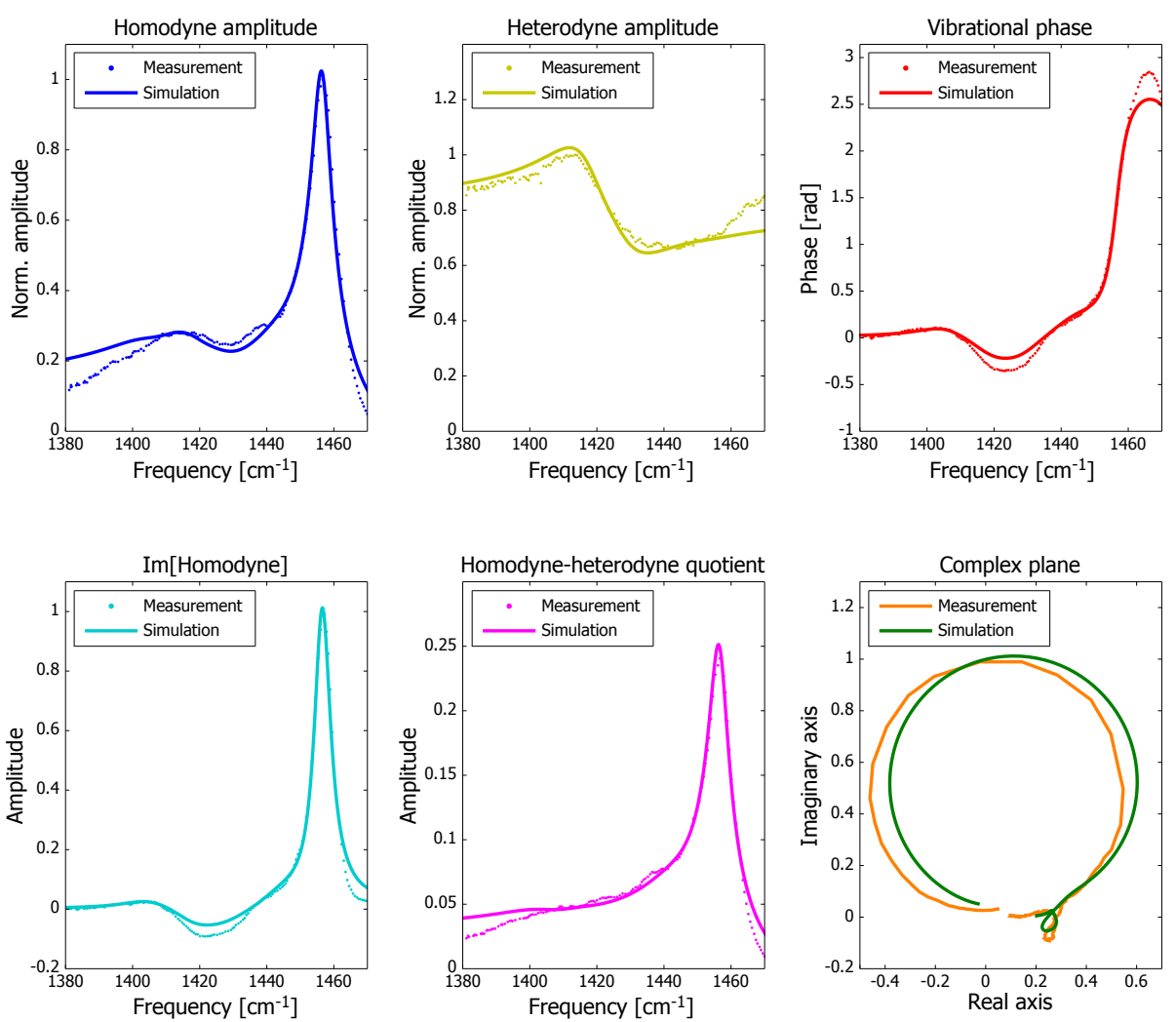

Figure 3.17. Measurements and simulations of a dual-frequency VPC-CARS spectrum of neat DMSO in the fingerprint region. A pair of closely-spaced resonances at $1417 \mathrm{~cm}^{-1}$ and $1426 \mathrm{~cm}^{-1}$ generate the heterodyne peak with the negative phase, while a single prominent resonance at $2915 \mathrm{~cm}^{-1}$, slightly modified by secondary resonances at $2876 \mathrm{~cm}^{-1}$ and $3004 \mathrm{~cm}^{-1}$, produces the large positive phase curve. Fits are made according to the model in the text, using published resonance data as initial fit parameters and optimizing.

the spectrum. The over-estimation of the amplitude on the low and high ends of the spectrum can be somewhat justified by imperfect correction of the data for changes in optical power on the sample as the OPO is tuned. The OPO output beams are observed to change spatial profile at different intracavity Lyot filter settings, an effect that cannot be fully mitigated with spatial filters on the optical table and is difficult to characterize at the sample; for some settings OPO settings the idler spatial mode even becomes a donut! However. even given that argument the heterodyne amplitude shows a marked shoulder that cannot be simulated using any of the peaks given in the literature and should 


\begin{tabular}{c|cc|cc|} 
& \multicolumn{2}{|c|}{ Data from Ref. $[126]$} & \multicolumn{2}{c|}{ Fit to data } \\
\hline Frequency $\left(\mathrm{cm}^{-1}\right)$ & Rel. int. & Width $\left(\mathrm{cm}^{-1}\right)$ & Rel. int. & Width $\left(\mathrm{cm}^{-1}\right)$ \\
\hline 3004 & 0.13 & 6.5 & 0.18 & 10 \\
2913 & 1.00 & 5.4 & 1.00 & 6 \\
2876 & 0.05 & 21.3 & 0.21 & 20 \\
2830 & 0.02 & 14.0 & 0.004 & 20 \\
2809 & 0.03 & 12.3 & 0.08 & 20 \\
1450 & 0.08 & 4.5 & 0.002 & 20 \\
1426 & 0.06 & 21.6 & 0.03 & 20 \\
1417 & 0.19 & 18.7 & 0.04 & 18 \\
1307 & 0.03 & 15.3 & 0.002 & 20 \\
\hline
\end{tabular}

Table 3.2. Comparison of the amplitudes and linewidths of DMSO from spontaneous Raman spectroscopy and from a fit to a dual-frequency VPC-CARS spectrum. The frequencies and non-resonant backgrounds are identical between the two parameter sets.

not be assumed to be a result of mischaracterization of the optical setup.

Further deviations from theory appear around the high-frequency resonance, which has a much larger experimental phase than can be achieved in simulations. We determined that this deviation is not caused by a systematic phase offset by measuring the signal from a piece of fully non-resonant glass. A relatively strong resonance in the low-frequency manifold near $1475 \mathrm{~cm}^{-1}$ may explain both the steep phase drop on the blue side of the high-frequency resonance as well as the extra amplitude in the heterodyne channel. The extra minor peak in both the homodyne and heterodyne channels at $1435 \mathrm{~cm}^{-1}$ likewise cannot be explained by changes in the pump field amplitudes. There are however no peaks observed to be at these frequency in spontaneous Raman scattering experiments, their appearance in VPC-CARS spectra is reproducible and consistent, and increasing the amplitude of the resonance at $1450 \mathrm{~cm}^{-1}$ in simulations does not improve the fit at either frequency. 



\section{CHAPTER 4}

\section{Vibrational molecular interferometry}

Heterodyne CARS has been described in semi-classical terms since its introduction. As a wave-mixing process where the molecule is merely an intermediate is has tremendous power, enabling the determination of the full complex vibrational response of a diffraction-limited region in a fraction of a second. However, it is a process that requires interferometric stability, even when the local phase is measured in real time with the heterodyne phase, and the alignment is tricky. A new paradigm for heterodyne CARS was presented by Rahav and Mukamel[17] in 2010. They proposed a fully quantum mechanical description of heterodyne CARS that focused on the molecule as the star of the show. This theoretical framework re-casts the interactions between the fields and the molecule in terms of dissipative and parametric processes. Dissipative processes are those in which the final state of the molecule is different than the initial state, and are directly related to the imaginary component of $\chi^{(3)}$. Parametric processes are those in which energy is simply re-arranged among the optical fields and the molecule is returned to its initial state at the end of the interaction. These interactions are correlated with the real component of $\chi^{(3)}$. This description of the optical interactions as dissipative and parametric processes is more transparent than the semi-classical field-mixing picture. A full picture of the nonlinear interactions can be gained by simply monitoring the amplitudes of all fields, and interferometric stability is not necessary. In this chapter we outline the theory behind this new description of heterodyne CARS and demonstrate fully background-free measurements of vibrational resonances.

\subsection{Theory}

The theory is quite involved, and interested readers are directed to a variety of publications that go into the details[17, 127-130]. The crux of the theory is that the vibrational resonances of a sample can be described as dissipative contributions to the total signal, which lie on top of a persistent and undesireable 


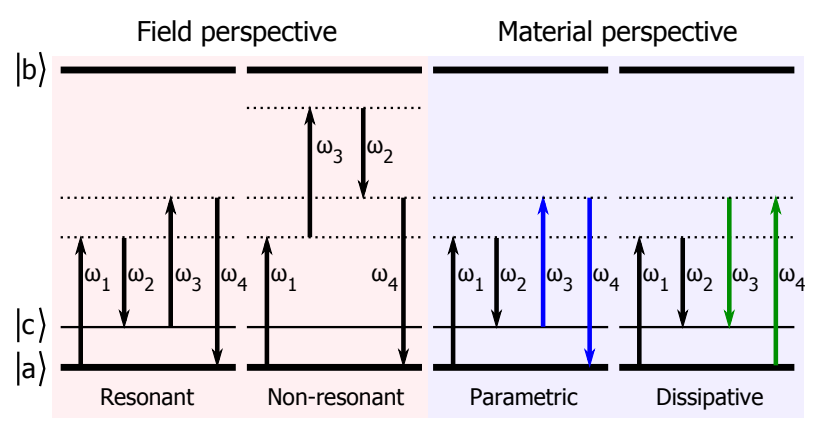

Figure 4.1. Energy level diagrams showing the field-mixing and material perspectives of heterodyne CARS. The field-mixing description of CARS includes resonant and non-resonant terms. In both cases the process ends with the molecule in the ground state, and the detected signal results from an emission at the anti-Stokes frequency. From the material perspective CARS is described in terms of parametric and dissipative processes, the latter of which leaves the molecule in a vibrational state and is the interference of two Stokes Raman transitions. The blue and green arrows emphasize the difference between the parametric and dissipative processes.

parametric background. To arrive at this result, we begin with a three-level material - consisting of ground state $|a\rangle$, lowest excited electronic state $|b\rangle$, and vibrational state $|c\rangle$-interacting with four incoming optical fields. The full Hamiltonian of this system is written as

$$
\mathscr{H}_{\text {tot }}=\mathscr{H}_{m}+\mathscr{H}_{f}+\mathscr{H}_{\text {int }}
$$

where the material Hamiltonian is

$$
\mathscr{H}_{m}=\hbar \omega_{a}|a\rangle\left\langle a\left|+\hbar \omega_{b}\right| b\right\rangle\left\langle b\left|+\hbar \omega_{c}\right| c\right\rangle\langle c|
$$

for all three states, the field Hamiltonian is

$$
\mathscr{H}_{f}=\sum_{i=1}^{4} \hbar \omega_{i} \hat{a}_{i}^{\dagger} a_{i}
$$


and the dipole coupling is given by

$$
\begin{aligned}
\mathscr{H}_{\text {int }} & =\left(\frac{2 \pi \omega_{1}}{\Omega}\right)^{1 / 2} \hat{a}_{1} e^{-i \omega_{1} t} \mu_{b a}|b\rangle\left\langle a\left|+\left(\frac{2 \pi \omega_{2}}{\Omega}\right)^{1 / 2} \hat{a}_{2} e^{-i \omega_{2} t} \mu_{b a}\right| b\right\rangle\langle c| \\
& +\left(\frac{2 \pi \omega_{3}}{\Omega}\right)^{1 / 2} \hat{a}_{3} e^{-i \omega_{3} t} \mu_{b a}|b\rangle\left\langle c\left|+\left(\frac{2 \pi \omega_{4}}{\Omega}\right)^{1 / 2} \hat{a}_{4} e^{-i \omega_{4} t} \mu_{b a}\right| b\right\rangle\langle a|+\text { h.c. }
\end{aligned}
$$

with the dipole moment of the transition from state $|a\rangle$ to state $|b\rangle$ given by $\mu_{b a}$, in quantization volume $\Omega$, driven by optical frequencies $\omega_{i}$. The energy difference between any two states $|m\rangle$ and $|n\rangle$ is $\hbar \omega_{m n}$. For a field in an initial coherent state $\left|\alpha_{s}\right\rangle$, the photon annihilation (creation) operator $\hat{a}_{i}\left(\hat{a}_{i}^{\dagger}\right)$ has an eigenvalue $\alpha_{s}\left(\alpha_{s}^{*}\right)$. The individual optical field operators can be written as

$$
\hat{\mathscr{E}}_{m}(t)=\left(\frac{2 \pi \omega_{m}}{\Omega}\right)^{1 / 2} \hat{a}_{m} e^{-i \omega_{m} t}
$$

so that the total electric field operator is

$$
\hat{E}(t)=\sum_{m=1}^{4} \hat{\mathscr{E}}_{m}(t)+\hat{\mathscr{E}}_{m}^{\dagger}(t) .
$$

For a field in an initially coherent state $|\Psi\rangle$ the expectation value of the electric field in Eq. 4.4 is

$$
E(t)=\langle\Psi|\hat{E}(t)| \Psi\rangle=\mathscr{E}(t)+c . c .
$$

where the absence of a hat indicates that $\mathscr{E}$ contains the eigenvalue $\alpha_{s}$ in place of the annihilation operator $\hat{a}$ in Eq. 4.3. This field can equivalently be written in the frequency domain as

$$
\begin{aligned}
E(\omega) & =\int E(t) e^{i \omega t} d t \\
& =2 \pi \sum_{m=1}^{4}\left[\mathscr{E}_{m} \delta_{\Delta}\left(\omega-\omega_{m}\right)+\mathscr{E}_{m}^{*} \delta_{\Delta}\left(\omega+\omega_{m}\right)\right]
\end{aligned}
$$

with the unusual term $\delta_{\Delta}$ representing a very narrow, Dirac delta-like function with non-zero width. This term is necessary to allow the duration of the electric field to be finite, i.e. to represent a laser pulse.

Given this field and the Hamiltonian in Eq. 4.1, the probability of a Ramanactive transition from the ground state $|a\rangle$ to the vibrational state $|c\rangle$, without 
striking electronic state $|b\rangle$ via a one-photon transition, is[17]

$$
\begin{aligned}
P_{a \rightarrow c} & =\left|\left\langle c\left|\mathscr{H}_{\text {tot }}\right| a\right\rangle\right|^{2} \\
& =\frac{1}{4 \pi^{2} \hbar^{4}}\left|\mu_{c b}\right|^{2}\left|\mu_{b a}\right|^{2}\left|\int \frac{E(\omega) E\left(\omega_{c a}-\omega\right)}{\omega-\omega_{b a}+i \eta} d \omega\right|^{2}
\end{aligned}
$$

Substituting Eq. 4.6 into Eq. 4.7 gives

$$
\begin{aligned}
P_{a \rightarrow c} \simeq & \frac{4 \pi^{2}}{\hbar^{4}\left|\mu_{c b}\right|^{2}\left|\mu_{b a}\right|^{2}} \\
\times & \mid \frac{\mathscr{E}_{1} \mathscr{E}_{2}^{*}}{\omega_{1}-\omega_{b a}+i \eta} \delta_{\Delta^{\prime}}\left(\omega_{1}-\omega_{2}-\omega_{c a}\right) \\
& \quad+\left.\frac{\mathscr{E}_{4} \mathscr{E}_{3}^{*}}{\omega_{4}-\omega_{b a}+i \eta} \delta_{\Delta^{\prime}}\left(\omega_{4}-\omega_{3}-\omega_{c a}\right)\right|^{2}
\end{aligned}
$$

which contains a narrow Dirac delta-like term $\delta_{\Delta^{\prime}}$ that is slightly different than that found in Eq. 4.6 due to the integral of Eq. 4.7. Expanding the right hand side of this equation via its square modulus,

$$
\begin{aligned}
P_{a \rightarrow c} & \simeq\left(\left|\frac{\mathscr{E}_{1} \mathscr{E}_{2}^{*}}{\omega_{1}-\omega_{b a}+i \eta}\right|^{2} \delta_{\Delta^{\prime}}^{2}\left(\omega_{1}-\omega_{2}-\omega_{c a}\right)\right. \\
& +\left|\frac{\mathscr{E}_{4} \mathscr{E}_{3}^{*}}{\omega_{4}-\omega_{b a}+i \eta}\right|^{2} \delta_{\Delta^{\prime}}^{2}\left(\omega_{4}-\omega_{3}-\omega_{c a}\right) \\
& +2 \Re\left[\frac{\mathscr{E}_{1} \mathscr{E}_{2}^{*} \mathscr{E}_{3} \mathscr{E}_{4}^{*}}{\left(\omega_{1}-\omega_{b a}+i \eta\right)\left(\omega_{4}-\omega_{b a}-i \eta\right)}\right] \\
& \left.\times \delta_{\Delta^{\prime}}\left(\omega_{1}-\omega_{2}-\omega_{c a}\right) \delta_{\Delta^{\prime}}\left(\omega_{4}-\omega_{3}-\omega_{c a}\right)\right) \frac{4 \pi^{2}}{\hbar^{4}}\left|\mu_{c b}\right|^{2}\left|\mu_{b a}\right|^{2} \\
& =P_{a \rightarrow c}^{12}+P_{a \rightarrow c}^{34}+P_{a \rightarrow c}^{1234}
\end{aligned}
$$

we arrive at an expression that has three distinct terms. The first two terms are pump-probe transitions into the vibrational state $|c\rangle$. The third term is an interference of these two pathways when $\omega_{1}-\omega_{2}=\omega_{4}-\omega_{3} \approx \omega_{c a}$, and is the only one that we will detect in our experimental configuration ${ }^{1}$. We define the

\footnotetext{
${ }^{1}$ The terms $\mathscr{E}_{1} \mathscr{E}_{2}^{*}$ and $\mathscr{E}_{4} \mathscr{E}_{3}^{*}$ oscillate at the vibrational frequency $\omega_{c a}$, which is much faster than we can detect, while $\mathscr{E}_{1} \mathscr{E}_{2}^{*} \mathscr{E}_{3} \mathscr{E}_{4}^{*}$ oscillates at megahertz rates; we use a similar modulation scheme here to the one that was described in the previous chapter.
} 
nonlinear susceptibility to be

$$
\chi^{(3)}=-\hbar^{-3} \frac{\left|\mu_{b a}\right|^{2}\left|\mu_{c b}\right|^{2}}{\left(\omega_{1}-\omega_{2}+\omega_{3}-\omega_{b a}+i \eta\right)\left(\omega_{1}-\omega_{2}-\omega_{c a}+i \eta\right)\left(\omega_{1}-\omega_{b a}+i \eta\right)}
$$

so that the amplitude of the transmitted field mode $S_{4}$ can be written as

$$
S_{4}=-\frac{4 \pi}{\hbar} \Im\left[\mathscr{E}_{1} \mathscr{E}_{2}^{*} \mathscr{E}_{3} \mathscr{E}_{4}^{*} \chi^{(3)}\left(-\omega_{4} ; \omega_{3},-\omega_{2}, \omega_{1}\right)\right] \delta\left(\omega_{1}-\omega_{2}+\omega_{3}-\omega_{4}\right)
$$

When all of these transitions are far from the nearest electronic resonance, the terms containing $\omega_{b a}$ are effectively real, while the term involving $\omega_{c a}$ is imaginary. Equation 4.11 can therefore be separated as

$$
S_{4}=-\frac{1}{2} P_{a \rightarrow c}^{1234}+S_{4}^{\text {par }}
$$

where the first term is associated with $\Im\left[\chi^{(3)}\right]$ and the second part is related to $\Re\left[\chi^{(3)}\right]$. In fact, all four field amplitudes can be written in this form, which then look like

$$
\begin{aligned}
S_{1} & =-\frac{1}{2} P_{a \rightarrow c}^{1234}-S_{1}^{\mathrm{par}} \\
S_{2} & =+\frac{1}{2} P_{a \rightarrow c}^{1234}+S_{2}^{\mathrm{par}} \\
S_{3} & =+\frac{1}{2} P_{a \rightarrow c}^{1234}-S_{3}^{\mathrm{par}} \\
S_{4} & =-\frac{1}{2} P_{a \rightarrow c}^{1234}+S_{4}^{\mathrm{par}} .
\end{aligned}
$$

Schematically, the physical processes that generate these terms are shown in Fig. 4.2(a) and (b). The prefactor of $1 / 2$ on the first terms in each line of Eqs. 4.13 results from the fact that only one of the two branches of the $P_{a \rightarrow c}^{1234}$ term affect the number of photons in each mode. The signs represent photon creation (+) and annhilation (-) processes. The detection of multiple field modes can allow the full rejection of all parametric processes. For example,

$$
\begin{aligned}
S_{4}-S_{2} & =\left[-\frac{1}{2} P_{a \rightarrow c}^{1234}+S_{4}^{\mathrm{par}}\right]-\left[+\frac{1}{2} P_{a \rightarrow c}^{1234}+S_{2}^{\mathrm{par}}\right] \\
& =-P_{a \rightarrow c}^{1234}
\end{aligned}
$$

which is the purely dissipative transition between the ground state $|a\rangle$ and 

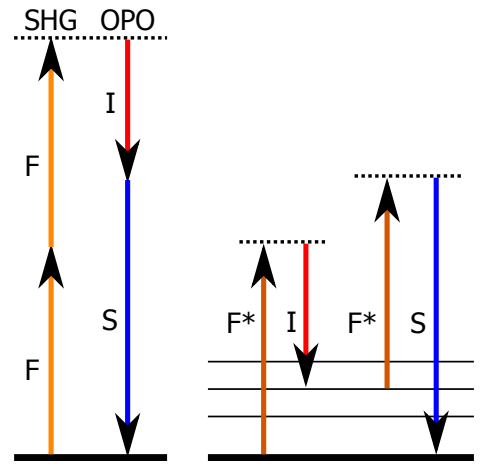

(a)

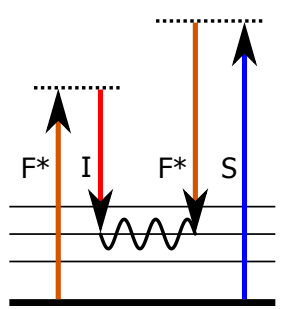

(b)

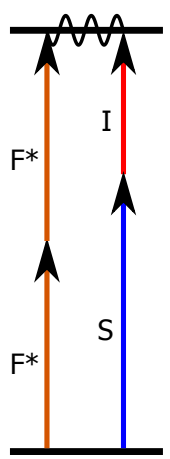

(c)

Figure 4.2. Energy level diagrams of the cascaded phase-preserving chain (left), parametric (a), vibrational dissipative (b), and electronic dissipative (c) energy transfer processes in the molecule. $F=$ laser fundamental at $1064 \mathrm{~nm}$, $F^{*}=$ frequency modulated laser fundamental, $S=$ OPO signal, and $I=$ OPO idler. The thick horizontal lines are electronic states, the thin horizontal lines are vibrational levels within an electronic state, and the dotted horizontal lines are virtual levels.

vibrational state $|c\rangle$.

An extension of this theory comes when two-photon electronic excitations are allowed. Up until this point it has been assumed that $\omega_{b a}$ is far higher than any individual optical field; that assumption will not change. However, we assume that, in addition to $\omega_{1}-\omega_{2}=\omega_{4}-\omega_{3} \approx \omega_{c a}$ there is another resonance such that $\omega_{1}+\omega_{3}=\omega_{2}+\omega_{4} \approx \omega_{b a}$. In this case, the terms in Eq. 4.13 can be re-written to include the electronic dissipative process

$$
\begin{aligned}
S_{1} & =-\frac{1}{2} P_{a \rightarrow c}^{1234}-S_{1}^{\mathrm{par}}-\frac{1}{2} P_{a \rightarrow b}^{1234} \\
S_{2} & =+\frac{1}{2} P_{a \rightarrow c}^{1234}+S_{2}^{\mathrm{par}}-\frac{1}{2} P_{a \rightarrow b}^{1234} \\
S_{3} & =+\frac{1}{2} P_{a \rightarrow c}^{1234}-S_{3}^{\mathrm{par}}-\frac{1}{2} P_{a \rightarrow b}^{1234} \\
S_{4} & =-\frac{1}{2} P_{a \rightarrow c}^{1234}+S_{4}^{\mathrm{par}}-\frac{1}{2} P_{a \rightarrow b}^{1234} .
\end{aligned}
$$

From this set of equations it can be seen that the measurement and addition of the field modes $S_{1}$ and $S_{3}$ yields information about both the dissipative component of the $a \rightarrow b$ transition and information about the parametric components. Therefore, the magnitudes of all of the optical processes can be determined by 
measuring all four fields and taking the sums and differences

$$
\begin{aligned}
S_{4}-S_{2} & =\left[-\frac{1}{2} P_{a \rightarrow c}^{1234}+2 S_{4}^{\mathrm{par}}-\frac{1}{2} P_{a \rightarrow b}^{1234}\right]-\left[+\frac{1}{2} P_{a \rightarrow c}^{1234}+2 S_{2}^{\mathrm{par}}-\frac{1}{2} P_{a \rightarrow b}^{1234}\right] \\
& =-P_{a \rightarrow c}^{1234} \\
S_{1}+S_{3} & =\left[-\frac{1}{2} P_{a \rightarrow c}^{1234}-S_{1}^{\mathrm{par}}-\frac{1}{2} P_{a \rightarrow b}^{1234}\right]+\left[+\frac{1}{2} P_{a \rightarrow c}^{1234}-S_{3}^{\mathrm{par}}-\frac{1}{2} P_{a \rightarrow b}^{1234}\right] \\
& =-S_{1}^{\mathrm{par}}-S_{3}^{\mathrm{par}}-P_{a \rightarrow b}^{1234}
\end{aligned}
$$

The sum $S_{3}+S_{1}$ contains no information on the dissipative vibrational processes, but contains full information about the electronic states and the parametric terms. On the other hand, the difference between $S_{4}$ and $S_{2}$ is always zero on electronic resonance. Therefore, by carefully determining the amplitude gain and loss in each field we can paint a full picture of the parametric and dissipative terms for both electronic and vibrational levels. We refer to this process, in which both electronic and vibrational states can be simultaneously measured, as vibrational molecular interferometry (VMI).

\subsection{Experimental setup}

We use a very similar setup as that described in the previous chapter, with a few modifications, and due to the setup we cannot assume that $\omega_{1}$ and $\omega_{3}$ are independent fields. In our case they are identical, and are provided by the laser fundamental beam at $1064 \mathrm{~nm}$. The field $\omega_{2}<\omega_{1}$ is provided by the OPO idler beam, while $\omega_{4}>\omega_{1}$ is the OPO signal. Frequency-conservation is automatically maintained with our cascaded phase-preserving chain. In the early iteration of the setup, as described in Ref. [23], the laser fundamental was modulated at $500 \mathrm{kHz}$ above the laser repetition rate with a phase-locked loop; the current iteration applies an 85.65 $\mathrm{MHz}$ modulation to the $\mathrm{AOM}$ and automatically detects the difference between this modulation and the laser repetition rate.

The most significant experimental change is the addition of optics and detectors for the laser fundamental and idler beams. A short-pass dichroic beamsplitter (ThorLabs FES1000) reflects the laser fundamental and idler beams and transmits the signal and anti-Stokes beams. To separate the idler and laser fundamental beams we installed a long-pass dichroic mirror (ThorLabs 
FEL1180). Further spectral cleaning is accomplished with dielectric filters. The idler is detected on reverse-biased InGaAs photodiode (ThorLabs FGA21, $-15 \mathrm{~V})$, while the laser fundamental is detected on a Si photodiode identical to that used for the signal beam.

Electrical signals from each photodiode are cleaned with electronic bandpass filters (Minicircuits BBP-10.7+), amplified (Minicircuits ZFL500LN+), and sent to high frequency lock-in amplifiers, with each photodiode getting its own channel. A fourth lock-in channel is reserved for a photomultiplier tube that measures the local phase. The in-phase and quadrature components of the signal photodiode and photomultiplier tube are individually sent to a data acquisition board, while only the amplitudes of the laser fundamental and idler channels are recorded. Custom LabVIEW software processes these inputs, along with calibrations and corrections for the different throughputs, sensitivities, and characteristics of the optics and detectors. The LabVIEW program displays by default the vibrational VMI (signal minus idler, $S_{4}-S_{2}$ ), electronic VMI (laser fundamental $\left(S_{1}+S_{3}\right)$, VPC-CARS amplitude, and VPCCARS phase simultaneously and in real time. If one of these modalities is not needed, it can be replaced with an amplitude-modulated detection technique (e.g. stimulated Raman loss (SRL) or gain (SRG), or modulated CARS as described in Chapter 2). If desired, the microscope control software can record an additional three channels: we typically look at the transmitted DC CARS signal, the epi-detected CARS or fluorescence signal, and the linear transmission of the signal beam.

Spectroscopy and microscopy are independently possible, or can be combined with hyperspectral scanning in an identical fashion to what was described in Chapter 2. Scan rates are limited by the analog input rate of the data acquisition board, which is currently capped at $1 \mathrm{Mpx} / \mathrm{s}$; for a six-channel experiment, the maximum attainable pixel update rate is about $170 \mathrm{kpx} / \mathrm{s}$. In practice, it is worthwhile to proceed at slower speeds to reduce electronic noise.

\subsection{Results}

\subsubsection{Resonant vibrational spectroscopy}

To demonstrate the full rejection of the parametric term we performed VMI, VPC-CARS, and traditional CARS spectroscopy on a sample of neat dimethyl sulfoxide (DMSO). A pair of symmetric methyl groups on DMSO allows two Raman-active vibrational modes in the high-frequency alkyl region: a dominant 


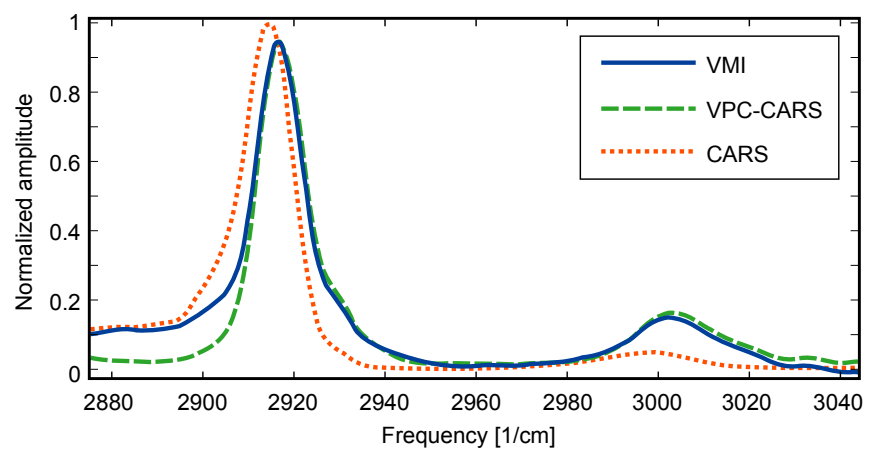

Figure 4.3. Vibrational specturm of neat DMSO measured with CARS, VPCCARS, and VMI. All spectra have been corrected for changes in the powers of the signal and idler beams. Note that the magnitude of the CARS spectrum represents a modulus-squared intensity rather than an amplitude.

feature at $2915 \mathrm{~cm}^{-1}$, and a weaker mode at $3005 \mathrm{~cm}^{-1}$. The ratio of the intensities of these two peaks in spontaneous Raman scattering has been measured in ambient conditions to be about 6:1. They are separated far enough that the wings of their resonant Lorentzian lineshapes do not overlap appreciably.

From Fig. 4.3 it is clear that there is a significant difference between the CARS spectrum and the VPC-CARS (here only the projection $\Im\left[\chi^{(3)}\right]$ is shown) and VMI spectra; the peaks of the former form approximate Fano profiles, the center frequencies of the vibrational modes are shifted lower, and the intensity ratio $I_{2915} / I_{3005}$ has increased to more than 30 . In contrast, the VMI and VPC-CARS spectra agree very well over most of the spectrum. Low optical power on the red side of the spectrum created significant noise and mode-hopping during the VMI scan, which was conducted independently of the VPC-CARS and CARS scans.

\subsubsection{Resonant vibrational microscopy}

For microscopy on a vibrationally resonant sample without electronic background we turn to mayonnaise, which is an emulsion of lipids in water, with a minority component of phospholipid and/or protein emulsifier. The two dominant components are the lipid, which has numerous resonances between $2820 \mathrm{~cm}^{-1}$ and $3030 \mathrm{~cm}^{-1}$, and water, which is almost completely non-resonant below $3000 \mathrm{~cm}^{-1}$. As a test of the rejection of the parametric component in a single-frequency microscopy measurement, this sample is nearly ideal. 

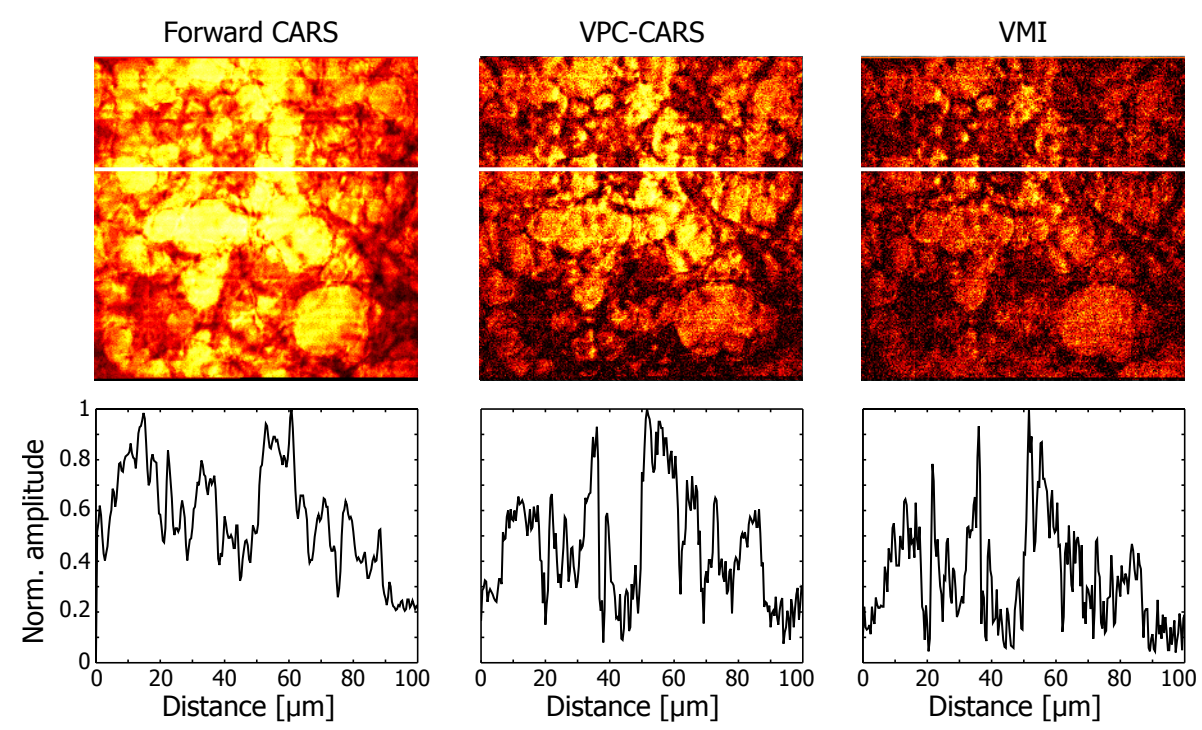

Figure 4.4. Images of mayonnaise recorded at $2845 \mathrm{~cm}^{-1}$. Transmission CARS (left) contains a significant background signal from water, which both VPC-CARS (center) and VMI (right) successfully suppress. The VPC-CARS image is the projection of the imaginary component. Intensity plots across the indicated line in the images are shown below their respective images.

Coherent Raman microscopy in transmission can generate large amounts of non-resonant background, as seen in Fig. 4.4(a). Although the lipids are still fairly easy to distinguish amongst the water background, the signal-tobackground contrast is only about 2.5. On the other hand, both VPC-CARSagain, only the imaginary component of $\chi^{(3)}$ is shown - and VMI show almost complete rejection of the water signal, so that the contrast-limiting factor is the noise of the measurement. This set of images was recorded simultaneously, covering $256 \times 256$ pixels in 4 seconds with a $25 \mu$ s lock-in time constant. The excess noise present in the VMI channel compared to the VPC-CARS channel is due to optical and electrical characteristics of the InGaAs photodiode: it has a higher capacitance, higher noise-equivalent-power, higher dark current, and receives less optical power than the signal and laser fundamental diodes. Even with the extra noise, the signal-to-noise ratio in the VMI image is estimated to be about three-fold better than in the CARS image. 


\subsubsection{Electrovibrational spectroscopy}

As a final test of VMI, we use a model example of a sample that is problematic for CARS. The laser dye DCM-pyran, whose absorption and emission spectra are shown in Fig. 4.5(a)[131], has two-photon-excited resonances for all three combinations of the signal and laser fundamental beams, and has a broad emission profile that covers the anti-Stokes wavelength range that we detect in alkyl CARS. The emission spectrum from a saturated solution of DCM-pyran in DMSO is shown in Fig. 4.5(b), and is dominated by fluorescence, masking even the strong $2915 \mathrm{~cm}^{-1}$ peak of DMSO. However, the vibrational VMI spectrum $\left(S_{4}-S_{2}\right)$ rejects this electronic background and the parametric vibrational process, revealing both the $2915 \mathrm{~cm}^{-1}$ peak and the weaker $3005 \mathrm{~cm}^{-1}$ peak while displaying only a small amount of residual electronic background signal from the DCM-pyran.

The amplitude of the $S_{1}+S_{3}$ signal (labeled "1064 nm" in Fig. 4.5(b)) shows net loss from the electronic resonance when detuned from the vibrational resonance, and suddenly gains a dispersive shape typical of the real component of $\chi^{(3)}$ when tuned onto the strong $2915 \mathrm{~cm}^{-1}$ resonance. Because the $1064 \mathrm{~nm}$ beam is degenerately used in both Stokes Raman processes in opposite roles - once as the pump field, and once as the Stokes field - the dissipative vibrational contribution to the amplitude of the field cancels out, leaving only $S_{3}+S_{1}=-2 S_{p a r}-P_{a \rightarrow b}^{1234}$, which are the purely real component of $\chi^{(3)}$ and the two-photon dissipative electronic transition. Incidentally, fluorescence resulting from a two-photon absorption is also proportional to the absorption cross-section. Therefore, the normalized magnitude of a resonant electronic two-photon absorption should be equal to the normalized fluorescent emission. We find that the relative magnitudes of the loss on the $1064 \mathrm{~nm}$ field and the fluorescence from the DCM-pyran agree very well over the entire spectra range, with the exception of the aforementioned DMSO vibrational resonance. Note that both of these processes are mediated by a two-photon absorption of the signal and idler fields, which results in the decreasing amplitude toward the low- and high-frequency edges of the window. 

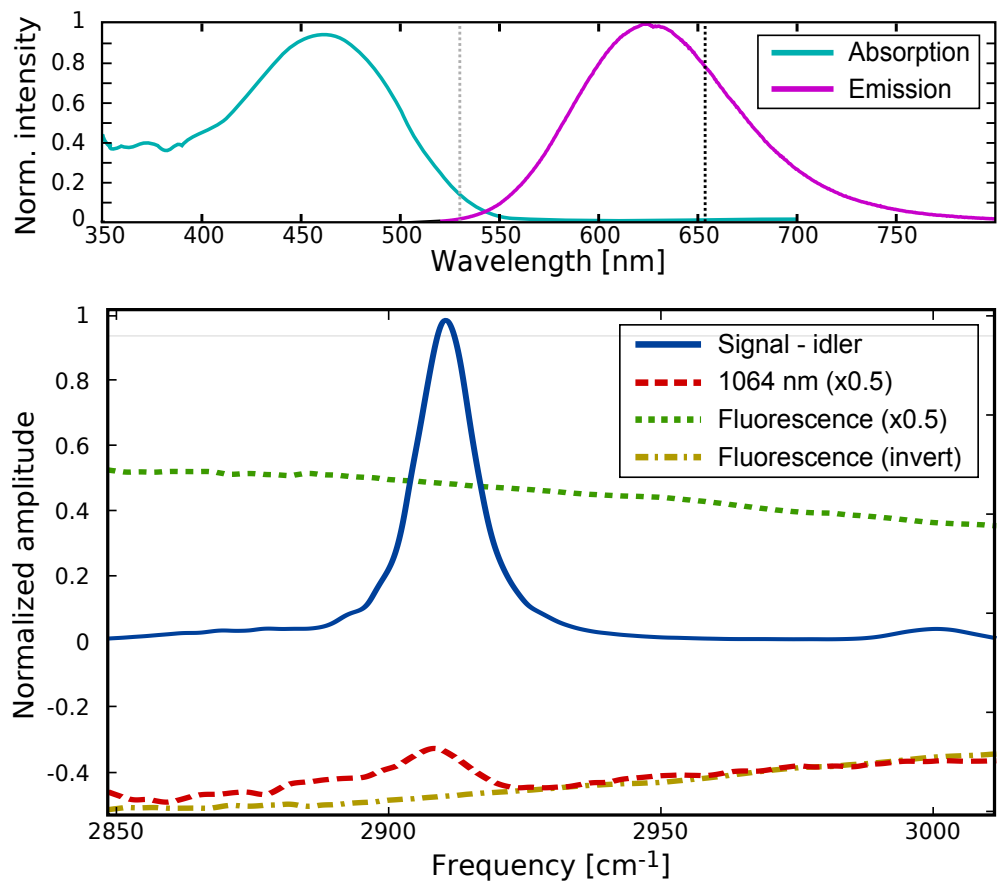

Figure 4.5. (a) Electronic absorption and emission spectra of DCM-pyran in DMSO. The left vertical line is the wavelength of the doubled laser fundamental (and the sum frequency of the signal and idler beams); the right vertical line is the approximate wavelength of the anti-Stokes emission at $2845 \mathrm{~cm}^{-1}$. (b) Simultaneous background-free vibrational and electronic measurements of DMSO saturated with DCM-pyran. Inverting the fluorescence measurement associates it with an absorption (loss) process, corresponding to the physical mechanism affecting the $1064 \mathrm{~nm}$ curve. None of the spectra have been corrected for optical power to illustrate the agreement between the fluorescence and $1064 \mathrm{~nm}$ profiles. 


\section{CHAPTER 5}

\section{Conclusions and outlook}

Throughout this thesis we have demonstrated methods for increasing the contrast of coherent Raman scattering experiments. In the most basic terms, CRS contrast is derived from the resonant motions of atoms relative to each other within molecules, but is fundamentally limited by a persistent non-resonant background. Removing, suppressing, or mitigating this background is the most straightforward path toward enhanced contrast. There are many techniques that have been developed to this end, and we have added three more to the CRS toolbox. The immediate application of two of these techniques has been the microscopic analysis and characterization of polymorphic materials, and we are confidant that others will soon follow.

Hyperspectral CARS microscopy combines the speed of narrowband imaging with the tunability of an optical parametric oscillator to rapidly acquire threedimensional spectral datacubes on a frame-by-frame basis, with each frame consisting of data at a single vibrational frequency, at rates exceeding two frames per second. Having spectral information at each pixel can remove the ambiguity that occurs during multi-spectral imaging, particularly when samples contain unexpected components. The technology is straightforward, mature, and robust, requiring minimal re-alignment and affording good reproducibility of measurements. The real power of the hyperspectral CARS system is in the data analysis. Realizing that CARS is an inherently nonlinear process, we forewent attempts at designing a quantitative analysis routine and instead designed a method for high-throughput qualitative interpretation of results. This method translates vibrational spectra into the visible by assigning unique colors to sequential vibrational frequencies based on color look-up tables. We have demonstrated that identifying multiple compounds in a heterogeneous mixture is straightforward even when those compounds are polymorphic and sensitive to orientation.

The main problem with any intensity-based CARS measurement is that the non-resonant background nonlinearly mixes with the resonant signal of inter- 
est, and disentangling these two components is difficult. While hyperspectral CARS somewhat alleviates this problem by allowing discrimination of the resonant and non-resonant components based on their spectral profiles, it is not a total solution. Instead we turn to phase-sensitive vibrational phase contrast CARS. A heterodyne measurement of the anti-Stokes field yields infomation about $\chi^{(3)}$ itself, rather than $\left|\chi^{(3)}\right|^{2}$ as in CARS, and is linear with the number of oscillators. However, the heterodyne signal contains a variable local background phase that is non-uniform across the field of view and fluctuates in time. Removing this local phase was the initial design of VPC-CARS, but was described only in phenomological terms. Measurements in the fingerprint region of the vibrational spectrum revealed that additional vibrational modes from a different manifold of the spectrum were being detected. We developed a mathematical treatment to describe these extra signals, and discovered that they originate from two vibrational manifolds actively coupling to each other via a homodyne interference process. When one of the manifolds is far from resonance its contribution to the total homodyne signal effectively disappears; it was serendipitous that the original VPC-CARS measurements succeeded at all!

With the new theory for the homodyne signal of VPC-CARS we have opened up a number of new experimental topics. Spectroscopy and microscopy in the fingerprint region can now be performed, albeit with more effort than required for high-frequency VPC-CARS. More intriguingly, we find that vibrational modes that are at nearly exact harmonic natural frequencies of each other can interfere constructively and generate a much stronger signal than either mode by itself. This enhancement cannot be generated by overlapping resonances within a single vibrational manifold, and is likely a path toward the sensitive detection of low concentrations of resonant molecules. However, this approach should be eyed warily; the vibrational modes in the two manifolds can destructively interfere for certain combinations of vibrational frequencies, reducing the overall sensitivity of the experiment. Proper selection of the minority and majority molecules is necessary to obtain a benefit from this interference. For example, for fingerprint bands that correspond to alkyl or hydroxyl groups in the high-frequency manifold there are many options available for the majority component.

Advances in electronics and software opened the way to high-speed VPCCARS microscopy. We detect the heterodyne channel signal on a simple $50-\Omega$ terminated photodiode with a high-power local oscillator, and are able to image at up to $200 \mathrm{kpx} / \mathrm{s}$, an improvement of over two orders of magnitude from 
the previous setup. The new VPC-CARS system is fully compatible with the hyperspectral software, enabling hyperspectral VPC-CARS microscopy within reasonable times. The transition to a large-area detector and high local oscillator power has also enabled VPC-CARS in a reflection configuration, opening up possibilities for imaging thick or strongly scattering samples.

The field-mixing picture that we typically use to describe VPC-CARS has a fault in that a local oscillator is designated from the beginning, and not all fields in the experiment are treated on the same footing. A new paradigm introduced theoretically by Rahav and Mukamel[17] demonstrated that analyzing the field-matter interaction from the perspective of the molecule simplifies the experimental configuration and the analysis of the data. We constructed an experiment to verify this theory, in which the process of interest-normally thought of as a resonant anti-Stokes emission - is in fact a pair of dissipative Stokes Raman pathways interfering at the molecule. Measuring the rates of photon annihilation and creation on only two of the incident fields provides us with enough information to exactly determine the dissipative contribution to the total signal, confirming that fully background-free vibrational microscopy and spectroscopy are possible without invoking arguments about heterodyne and homodyne phases and interferometric stability requirements. Surprisingly, this capability exists even in the presence of strong electronic resonances, a fact we confirmed by easily measuring the vibrational resonances of a liquid saturated with a laser dye. The fluorescence from the dye overpowered the anti-Stokes signal, but was nullified in the dual-field detection scheme. Furthermore, though not directly predicted by theory, we found that observing all of the fields in the process provided us with full information about both the vibrational and electronic resonances. We demonstrated simultaneous acquisition of the dissipative and parametric vibrational processes along with the electronic dissipative process and found good agreement with theory.

While the experimental configuration of VMI is in principle more straightforward than that of VPC-CARS, the requirement for detecting optical fields spanning nearly an octave presents difficulties. The most significant are in the collection and relay optics - which are optimized for visible transmission and have poor performance at idler wavelengths - and in the mid-infrared detectors, which have higher intrinsic noise and lower bandwidths than the silicon photodiodes used for the signal and laser fundamental beams. This combination of optical characteristics results in extra noise being added to VMI signals that is not present in either VPC-CARS or stimulated Raman scattering signals. New optics are being commercialized that include extended transmission in the mid- 
infrared, and detectors are steadily improving, so while VMI is currently not feasible for many applications it may yet find a niche role.

The field of coherent Raman scattering microscopy has progressed rapidly over the past decade and a half, and the future looks bright. Challenges naturally still remain, in particular when it comes to interpreting CARS data. The non-resonant background intrinsically reduces contrast and generates artifacts that appear inexplicable to non-experts. While removing this background is ideal, the equipment required to do so is not trivial. The alternative that we propose is the qualitative representation of the spectral information as colors. We have found that training a user to interpret and understand the results of the spectral projection method takes less than an hour, and skilled users can parse data sets quicker than they can be acquired. Adding hyperspectral capabilities to more advanced techniques like VPC-CARS rapidly increases their utility across a broad field of applications, and entirely new paradigms allow the use of CRS methods on samples that have so far been considered inaccessible due to electronic backgrounds. 


\section{CHAPTER 6}

\section{Acknowledgments}

The book in your hands is a product of hard work put forth not only by the author but by a tremendous network of friendly, intelligent, and motivated people. Giving all of them the proper thanks for their respective contributions would require another book in and of itself.

First I want to thank my promoter and professor. Jennifer, I am grateful that you accepted me into the Optical Sciences group when I was an undergraduate, and that you let me continue here as a graduate student. You have been a constant source of inspiration and encouragement for me, and your support for both my scientific and personal endeavors has been immense. From you I have learned what it means to be a good scientist, a strong leader, and an excellent teacher. You also taught me that scientists, leaders, and teachers should love making and eating pancakes, and should not be afraid to sing every now and then.

Herman, looking back, I am truly amazed at how much freedom you gave me to pursue my own research directions. At times it was a bit chaotic, but in the end it all worked out pretty well. The open door to your office was always inviting, and even given how busy you are you have always had time to talk with me. Your wealth of knowledge is really mind-boggling. Whenever I had a problem, you had at least one answer. Whenever I was stuck, you had an idea. Perhaps most important, whenever I had a success, you had a critical temperance. One day you will see that single molecule.

This thesis probably would not have made it very far without the amazing technical skills of Jeroen Korterik. I probably won't ever have the grasp on noise that you do, letalone be able to understand the intricacies of high-frequency electronic theory or hardware-timed data acquisition. Time and again you helped me out by taking my ideas, some of which were borderline crazy, turning them into working solutions in record time, and explaining - often more than once - exactly how the new device worked. I'm glad that we also had a friendship beyond the technical stuff; there are few people with whom I would 
rather discuss the finer points of whiskey, bacon, and guitar amplifiers.

While my mailing address has changed quite a few times since I moved to Enschede, I have always felt at home in OS. No small part of that is due to the positive social dynamic that exists in the group. Having attended over a halfdozen group retreats in places as far-flung as Erlangen, Lund, and Giethoorn, not to mention countless barbecues, outings, and beer+1-'o-clocks, OS feels a bit more like a family than a research group to me. Frans, Jorick, Florian, Sven, Jesse, Dirk-Jan, Simon, Sean, Lourens, Stefan, you guys have been great fun to spend time with and talk to, no matter the topic, the hour, the volume of the music, or the par of the golf course. Thanks also to Roza, Dan, Qing, Ron, Meindert, Jinfeng, Sonia, Annemarie, Mustafa, Sergio, Yeng-Sheng, Gerwin, Bente, Rick, Divya, David, and Jeffrey for making OS such an interesting group. Andrew, you clued me in to magic that is pharmacy and provided invaluable input with the hyperspectral system. Frater, your green thumb paid off in a really big way. Doing that experiment was a lot of fun and turned out to be really scientifically interesting too! Martin, your patience while training me on the CARS setup paid off, and I am grateful for the time you spent during my master's project showing me the ropes.

Swing dancing has become a way of life for me whenever I can find time away from the lab, and I have had a fantastic time with everyone in Swing Out Loud. Teaching and dancing with all of you for the past three-odd years has been wonderful. Irene, I am glad that you started SOL and invited me to be a part of it. Jolet, you have been a great teaching partner, and you are one of the most dependable people I have ever known! Linnartz and Riëlle, thank you for your enthusiasm with all of the routines.

I want to thank all of the members of my committee who put in the time and effort to critically read through my thesis and offer suggestions for improvements. Cees, your input throughout my doctorate has been very helpful, and I particularly appreciate the effort that you put into carefully proofreading my papers. I especially want to extend my gratitute to Prof. John Brandenberger, for whom this is the second time serving on a thesis committee of mine. The lessons that you taught me as an undergraduate still guide my path to this day, and I find myself channeling your enthusiasm and passion to my own students.

Finally, to my family, who have been unceasingly supportive and encouraging. You have never been far from my thoughts. 


\section{Bibliography}

[1] E. Hecht, Optics. Addison-Wesley, third ed., 1998.

[2] J. Kepler, "Demonstratio eorum quae visui et visibilibus propter conspicilla non ita pridem inventa accidunt," Dioptrica, pp. 519-567, 1611.

[3] F. H. Wenham, "On the illumination of transparent microscopic objects on a new principle," Transactions of the Microscopy Society of London, vol. 3, pp. 83-90, 1850.

[4] F. Zernike, "Phase-contrast, a new method for microscopic observation of transparent objects.," Physica, vol. 9, pp. 974-986, 1942.

[5] A. Smekal, "Zur quantentheorie der dispersion," Naturwissenschaften, vol. 11, pp. 873875, 1923.

[6] V. V. Raman and K. S. Krishnan, "A new type of secondary radiation," Nature, vol. 121, pp. 501-502, 1928.

[7] E. Smith and G. Dent, Modern Raman spectroscopy: a practical approach. John Wiley \& Sons, 2005.

[8] J. Hilderink, C. Otto, C. Slump, A. Lenferink, M. Engelse, C. van Blitterswijk, E. de Koning, M. Karperien, and A. van Apeldoorn, "Label-free detection of insulin and glucagon within human Islets of Langerhans using Raman spectroscopy," PLoS ONE, vol. 8, pp. e78148-1-11, October 2013.

[9] M. D. Levenson and J. J. Song, Coherent Raman spectroscopy, vol. 21 of Topics in Current Physics, ch. 7, pp. 293-371. Springer Berlin Heidelberg, 1980.

[10] P. D. Maker and R. W. Terhune, "Study of optical effects due to an induced polarization third order in the electric field strength," Physical Review, vol. 137, pp. A801-818, February 1965.

[11] M. Cui, B. R. Bachler, and J. P. Ogilvie, "Comparing coherent and spontaneous Raman scattering under biological imaging conditions," Optics Letters, vol. 34, pp. 773-775, March 2009.

[12] P. F. Chimento, M. Jurna, H. S. P. Bouwmans, E. T. Garbacik, L. Hartsuiker, C. Otto, J. L. Herek, and H. L. Offerhaus, "High-resolution narrowband CARS spectroscopy in the spectral fingerprint region," J. Raman Spectrosc., 2009.

[13] E. O. Potma, C. L. Evans, and X. S. Xie, "Heterodyne coherent anti-Stokes Raman scattering (CARS) imaging," Opt. Lett., vol. 31, no. 2, pp. 241-243, 2006.

[14] M. Jurna, J. P. Korterik, C. Otto, J. L. Herek, and H. L. Offerhaus, "Vibrational phase contrast microscopy by use of coherent anti-Stokes Raman scattering," Phys. 
Rev. Lett., vol. 103, pp. 043905-(1-3), 2009.

[15] G. Lucassen, Polarization sensitive coherent Raman spectroscopy on (bio)molecules in solutions. PhD thesis, University of Twente, 1992.

[16] M. Jurna, Vibrational phase contrast CARS microscopy. PhD thesis, University of Twente, 2010.

[17] S. Rahav and S. Mukamel, "Stimulated coherent anti-Stokes Raman spectroscopy (CARS) resonances originate from double-slit interference of two-photon Stokes pathways," Proc. Natl. Acad. Sci. U. S. A., vol. 107, pp. 4825-4829, January 2010.

[18] E. T. Garbacik, J. L. Herek, C. Otto, and H. L. Offerhaus, "Rapid identification of heterogeneous mixture components with hyperspectral coherent anti-Stokes Raman scattering imaging," Journal of Raman Spectroscopy, vol. 43, pp. 651-655, 2012.

[19] E. T. Garbacik, A. L. Fussell, S. Gres, J. P. Korterik, C. Otto, J. L. Herek, and H. L. Offerhaus, "Hyperspectral coherent anti-Stokes Raman scattering microscopy for in situ analysis of solid-state crystal polymorphs," Proc. SPIE, vol. 8588, pp. 85880O-1$11,2013$.

[20] E. T. Garbacik, R. P. Korai, E. H. Frater, J. P. Korterik, C. Otto, and H. L. Offerhaus, "In planta imaging of $\delta^{9}$-tetrahydrocannabinolic acid in cannabis sativa $l$. with hyperspectral coherent anti-Stokes Raman scattering microscopy," Journal of Biomedical Optics, vol. 18, pp. 046009-1-5, 2013.

[21] M. Jurna, E. T. Garbacik, J. P. Korterik, J. L. Herek, C. Otto, and H. L. Offerhaus, "Visualizing resonances in the complex plane with vibrational phase contrast CARS," Anal. Chem., vol. 82, no. 16, pp. 7656-7659, 2010.

[22] A. Zare and P. Gader, "Sparsity promoting iterated constrained endmember detection with integrated band selection," IEEE Geoscience and Remote Sensing Letters, vol. 4, pp. 1-4, July 2007.

[23] E. T. Garbacik, J. P. Korterik, C. Otto, S. Mukamel, J. L. Herek, and H. L. Offerhaus, "Background-free nonlinear microspectroscopy with vibrational molecular interferometry," Physical Review Letters, vol. 107, pp. 253902-1-4, December 2011.

[24] E. T. Garbacik, J. P. Korterik, C. Otto, S. Mukamel, J. L. Herek, and H. L. Offerhaus, "Background-free nonlinear microspectroscopy with vibrational molecular interferometry," Proc. SPIE, vol. 8226, pp. 822605-1-7, 2012.

[25] M. D. Duncan, J. Reintjes, and T. J. Manuccia, "Scanning coherent anti-stokes raman microscope," Optics Letters, vol. 7, pp. 350-352, August 1982.

[26] A. Zumbusch, G. R. Holtom, and X. S. Xie, "Three-dimensional vibrational imaging by coherent anti-Stokes Raman scattering," Phys. Rev. Lett., vol. 82, no. 20, pp. 41424145, 1999.

[27] E. O. Potma, W. P. de Boeij, P. J. M. van Haastert, and D. A. Wiersma, "Real-time visualization of intracellular hydrodynamics in single living cells," Proceedings of the 
National Academy of Sciences, vol. 98, pp. 1577-1582, February 2001.

[28] J.-X. Cheng, Y. K. Jia, G. Zheng, and X. S. Xie, "Laser-scanning coherent anti-Stokes Raman scattering microscopy and applications to cell biology," Biophysical Journal, vol. 83, pp. 502-209, July 2002.

[29] C. L. Evans, E. O. Potma, M. Puoris'haag, D. Côté, C. P. Lin, and X. S. Xie, "Chemical imaging of tissue in vivo with video-rate coherent anti-stokes raman scattering microscopy," Proc. Natl. Acad. Sci. U. S. A., vol. 102, no. 46, pp. 16807-16812, 2005.

[30] S. A. Akhmanov, A. F. Bunkin, S. G. Ivanov, and N. I. Koroteev, "Coherent ellipsometry of Raman scattering of light," JETP Lett., vol. 25, p. 416, 1977.

[31] S. A. Akhmanov, A. F. Bunkin, S. G. Ivanov, and N. I. Koroteev, "Polarization active Raman spectroscopy and coherent Raman ellipsometry," Soviet Physics JETP, vol. 47, p. $667,1978$.

[32] A. Chikishev, G. Lucassen, N. Koroteev, C. Otto, and J. Greve, "Polarization sensitive coherent anti-Stokes Raman scattering spectroscopy of the amide I band of proteins in solutions," Biophys. J., vol. 63, pp. 976-985, October 1992.

[33] A. Voroshilov, C. Otto, and J. Greve, "On the coherent vibrational phase in polarization sensitive resonance CARS spectroscopy of copper tetraphenylporphyrin," Journal of Chemical Physics, vol. 106, pp. 2589-2598, February 1997.

[34] J.-X. Cheng, L. D. Book, and X. S. Xie, "Polarization coherent anti-Stokes Raman scattering microscopy," Opt. Lett., vol. 26, no. 17, pp. 1341-1343, 2001.

[35] K. Orsel, E. T. Garbacik, M. Jurna, J. P. Korterik, C. Otto, J. L. Herek, and H. L. Offerhaus, "Heterodyne interferometric polarization coherent anti-Stokes Raman scattering (HIP-CARS) spectroscopy," J. Raman Spectrosc., vol. 41, no. 12, pp. 1678-1681, 2010.

[36] F. Munhoz, Polarization resolved four-wave mixing microscopy: structural and vibrational read-out in molecular media. PhD thesis, Universite Paul Cezanne - Aix Marseille, 2010.

[37] F. Munhoz, H. Rigneault, and S. Brasselet, "Polarization-resolved four-wave mixing microscopy for structural imaging in thick tissues," Journal of the Optical Society of America B, vol. 29, pp. 1541-1550, June 2012.

[38] A. Laubereau and W. Kaiser, "Vibrational dynamics of liquids and solids investigated by picosecond light pulses," Reviews of Modern Physics, vol. 50, pp. 607-665, July 1978.

[39] A. Volkmer, L. D. Book, and X. S. Xie, "Time-resolved coherent anti-Stokes Raman scattering microscopy: Imaging based on Raman free induction decay," Appl. Phys. Lett., vol. 80, pp. 1505-1507, 2002.

[40] D. A. Sidorov-Biryukov, E. E. Serebryannikov, and A. M. Zheltikov, "Time-resolved coherent anti-Stokes Raman scattering with a femtosecond soliton output of a photonic- 
crystal fiber," Optics Letters, vol. 31, pp. 2323-2325, August 2006.

[41] B. von Vacano and M. Motzkus, "Time-resolving molecular vibration for microanalytics: single laser beam nonlinear raman spectroscopy in simulation and experiment," Phys. Chem. Chem. Phys., vol. 10, pp. 681-691, 2008.

[42] R. Selm, M. Winterhalder, A. Zumbusch, G. Krauss, T. Hanke, A. Sell, and A. Leitenstorfer, "Ultrabroadband background-free coherent anti-Stokes Raman scattering microscopy based on a compact Er:fiber laser system," Optics Letters, vol. 35, pp. 32823284 , Oct 2010.

[43] J.-X. Cheng, A. Volkmer, and X. S. Xie, "Theoretical and experimental characterization of coherent anti-Stokes Raman scattering microscopy," Journal of the Optical Society of America B, vol. 19, no. 6, pp. 1363-1375, 2002.

[44] C. Heinrich, S. Bernet, and M. Ritsch-Marte, "Wide-field coherent anti-Stokes Raman scattering microscopy," Applied Physics Letters, vol. 84, pp. 816-818, February 2004.

[45] M. Lei, M. Winterhalder, R. Selm, and A. Zumbusch, "Video-rate wide-field coherent anti-Stokes Raman scattering microscopy with collinear nonphase-matching illumination," Journal of Biomedical Optics, vol. 16, pp. 021102-1-5, February 2011.

[46] M. Jurna, J. P. Korterik, C. Otto, J. L. Herek, and H. L. Offerhaus, "Background free CARS imaging by phase sensitive heterodyne CARS," Opt. Express, vol. 16, no. 20, pp. 15863-15869, 2008.

[47] X. Wang, A. Zhang, M. Zhi, A. V. Sokolov, G. R. Welch, and M. O. Scully, "Heterodyne coherent anti-Stokes Raman scattering for spectral phase retrieval and signal amplification," Optics Letters, vol. 35, pp. 721-723, March 2010.

[48] B. Li, W. S. Warren, and M. C. Fischer, "Phase-cycling coherent anti-Stokes Raman scattering using shaped femtosecond laser pulses," Optics Express, vol. 18, pp. 2582525832, December 2010.

[49] D. Oron, N. Dudovich, D. Yelin, and Y. Silberberg, "Quantum control of coherent anti-Stokes Raman processes," Physical Review A, vol. 65, p. 043408, 2002.

[50] T. Hellerer, A. M. K. Enejder, and A. Zumbusch, "Spectral focusing: High spectral resolution spectroscopy with broad-bandwidth laser pulses," Appl. Phys. Lett., vol. 85, pp. 25-27, 2004.

[51] I. Rocha-Mendoza, W. Langbein, and P. Borri, "Coherent anti-Stokes Raman microspectroscopy using spectral focusing with glass dispersion," Applied Physics Letters, vol. 93, pp. 201103-1-3, November 2008.

[52] A. C. W. van Rhijn, H. L. Offerhaus, P. van der Walle, J. L. Herek, and A. Jafarpour, "Exploring, tailoring, and traversing the solution landscape of a phase-shaped CARS process," Opt. Express, vol. 18, no. 3, pp. 2695-2709, 2010.

[53] F. Ganikhanov, C. L. Evans, B. G. Saar, and X. S. Xie, "High-sensitivity vibrational imaging with frequency modulation coherent anti-Stokes Raman scattering (FM 
CARS) microscopy," Optics Letters, vol. 31, pp. 1872-1874, June 2006.

[54] B.-C. Chen, J. Sung, and S.-H. Lim, "Chemical imaging with frequency modulation coherent anti-Stokes Raman scattering microscopy at the vibrational fingerprint region," Journal of Physical Chemistry B, vol. 114, pp. 16871-16880, 2010.

[55] M. Windbergs, M. Jurna, H. L. Offerhaus, J. L. Herek, P. Kleinebudde, and C. J. Strachan, "Chemical imaging of oral solid dosage forms and changes upon dissolution using coherent anti-Stokes Raman scattering microscopy," Anal. Chem., vol. 81, no. 6, pp. 2085-2091, 2009.

[56] R. Leonhardt, W. Holzapfel, W. Zinth, and W. Raiser, "Terahertz quantum beats in molecular liquids," Chemical Physics Letters, vol. 133, pp. 373-377, January 1987.

[57] M. O. Scully, G. W. Kattawar, R. P. Lucht, T. Opatrny, H. Pilloff, A. Rebane, A. V. Sokolov, and M. S. Zubairy, "FAST CARS: Engineering a laser spectroscopic technique for rapid identification of bacterial spores," Proceedings of the National Academy of Sciences, vol. 99, pp. 10994-11001, 2002.

[58] J.-x. Cheng, A. Volkmer, L. D. Book, and X. S. Xie, "Multiplex coherent anti-Stokes Raman scattering microspectroscopy and study of lipid vesicles," J. Phys. Chem. B, vol. 106, no. 34, pp. 8493-8498, 2002.

[59] A. Rebane, M. Drobizhev, M. Kruk, A. Karotki, I. Tehver, and M. Scully, "Femtosecond resonance enhanced CARS for background-free detection of organic molecules," Journal of Modern Optics, vol. 52, no. 9, pp. 1243-1253, 2004.

[60] S. Postma, A. C. W. van Rhijn, J. P. Korterik, P. Gross, J. L. Herek, and H. L. Offerhaus, "Application of spectral phase shaping to high resolution CARS spectroscopy," Opt. Express, vol. 16, pp. 7985-7996, May 2008.

[61] D. Pestov, X. Wang, G. O. Ariunbold, R. K. Murawski, V. A. Sautenkov, A. Dogariu, A. V. Sokolov, , and M. O. Scully, "Single-shot detection of bacterial endospores via coherent Raman spectroscopy," Proceedings of the National Academy of Sciences, vol. 105, no. 2, pp. 422-427, 2008.

[62] M. Bonn, M. Mueller, H. A. Rinia, and K. N. Burger, "Imaging of chemical and physical state of individual cellular lipid droplets using multiplex CARS microscopy," Journal of Raman Spectroscopy, vol. 40, no. 7, pp. 763-769, 2009.

[63] A. C. W. van Rhijn, S. Postma, J. P. Korterik, J. L. Herek, and H. L. Offerhaus, "Chemically selective imaging by spectral phase shaping for broadband CARS around $3000 \mathrm{~cm}^{-1}$," Journal of the Optical Society of America B, vol. 26, pp. 559-563, March 2009.

[64] J. Rehbinder, C. Pohling, T. Buckup, and M. Motzkus, "Multiplex coherent anti-Stokes Raman microspectroscopy with tailored Stokes spectrum," Opt. Lett., vol. 35, no. 22, pp. 3721-3723, 2010.

[65] S. H. Parekh, Y. J. Lee, K. A. Aamer, and M. T. Cicerone, "Label-free cellular imaging 
by broadband coherent anti-Stokes Raman scattering microscopy," Biophysical Journal, vol. 99, pp. 2695-2704, 2010.

[66] C. M. Hartshorn, Y. J. Lee, J. Charles H. Camp, Z. Liu, J. Heddleston, N. Canfield, T. A. Rhodes, A. R. H. Walker, P. J. Marsac, and M. T. Cicerone, "Multicomponent chemical imaging of pharmaceutical solid dosage forms with broadband CARS microscopy," Analytical Chemistry, vol. 85, pp. 8102-8111, 2013.

[67] C.-Y. Chung, J. Hsu, S. Mukamel, and E. O. Potma, "Controlling stimulated coherent spectroscopy and microscopy by a position-dependent phase," Physical Review A, vol. 87, pp. 033833-1-6, 2013.

[68] G. W. H. Wurpel, H. A. Rinia, and M. Mueller, "Imaging orientational order and lipid density in multilamellar vesicles with multiplex CARS microscopy," Journal of Microscopy, vol. 218, pp. 37-45, April 2005.

[69] C. Brackmann, J. Norbeck, M. Akeson, D. Bosch, C. Larsson, L. Gustafsson, and A. Enejder, "CARS microscopy of lipid stores in yeast: the impact of nutritional state and genetic background," J. Raman Spectrosc., vol. 40, no. 7, pp. 748-756, 2009.

[70] A. Enejder, C. Brackmann, and F. Svedberg, "Coherent anti-Stokes Raman scattering microscopy of cellular lipid storage," IEEE Journal of Selected Topics in Quantum Electronics, vol. 16, pp. 506-515, June 2010.

[71] P. Chumnanpuen, C. Brackmann, S. K. Nandy, S. Chatzipapadopoulos, J. Nielsen, and A. Enejder, "Lipid biosynthesis monitored at the single-cell level in saccharomyces cerevisiae," Biotechnology Journal, vol. 6, pp. 1-8, 2011.

[72] W. Dou, D. Zhang, Y. Jung, J.-X. Cheng, and D. M. Umulis, "Label-free imaging of lipid-droplet intracellular motion in early drosophila embryos using femtosecondstimulated Raman loss microscopy," Biophysical Journal, vol. 102, pp. 1666-1675, April 2012.

[73] S. Yue, J. M. Crdenas-Mora, L. S. Chaboub, S. A. Lelivre, and J.-X. Cheng, "Labelfree analysis of breast tissue polarity by Raman imaging of lipid phase," Biophysical Journal, vol. 102, pp. 1215-1223, March 2012.

[74] Y. Fu, H. Wang, T. B. Huff, R. Shi, and J.-X. Cheng, "Coherent anti-Stokes Raman scattering imaging of myelin degradation reveals a calcium-dependent pathway in LysoPtdCho-induced demyelination," Journal of Neuroscience Research, vol. 85, pp. 28702881, June 2007.

[75] E. Belanger, S. Begin, S. Laffray, Y. D. Koninck, R. Vallee, and D. Cote, "Quantitative myelin imaging with coherent anti-Stokes Raman scattering microscopy: alleviating the excitation polarization dependence with circularly polarized laser beams," Opt. Express, vol. 17, no. 21, pp. 18419-18432, 2009.

[76] J. Imitola, D. Côté, S. Rasmussen, X. S. Xie, Y. Liu, T. Chitnis, R. L. Sidman, C. P. Lin, and S. J. Khourya, "Multimodal coherent anti-Stokes Raman scattering 
microscopy reveals microglia-associated myelin and axonal dysfunction in multiple sclerosis-like lesions in mice," Journal of Biomedical Optics, vol. 16, pp. 021109-111, February 2011.

[77] T. B. Huff, Y. Shi, W. Sun, W. Wu, R. Shi, and J.-X. Cheng, "Real-time CARS imaging reveals a calpain-dependent pathway for paranodal myelin retraction during high-frequency stimulation," PLoS ONE, vol. 6, pp. e17176-1-11, March 2011.

[78] N. M. Perney, L. Braddick, M. Jurna, E. T. Garbacik, H. L. Offerhaus, L. C. Serpell, E. Blanch, L. Holden-Dye, W. S. Brocklesby, and T. Melvin, "Polyglutamine aggregate structure in vitro and in vivo; new avenues for coherent anti-Stokes Raman scattering microscopy," PLoS ONE, vol. 7, pp. e40536-1-9, July 2012.

[79] N. L. Garrett, A. Lalatsa, I. Uchegbu, A. Schaetzlein, and J. Moger, "Exploring uptake mechanisms of oral nanomedicines using multimodal nonlinear optical microscopy," Journal of Biophotonics, vol. 5, pp. 458-468, March 2012.

[80] A. Fussell, E. Garbacik, H. Offerhaus, P. Kleinebudde, and C. Strachan, "In situ dissolution analysis using coherent anti-Stokes Raman scattering (CARS) and hyperspectral CARS microscopy," European Journal of Pharmaceutics and Biopharmaceutics, vol. 85, pp. 1141-1147, 2013.

[81] J. Sung, B.-C. Chen, and S.-H. Lim, "Fast three-dimensional chemical imaging by interferometric multiplex coherent anti-stokes raman scattering microscopy," J. Raman Spectrosc., vol. 42, no. 2, pp. 130-136, 2011.

[82] S. Bégin, B. Burgoyne, V. Mercier, A. Villeneuve, R. Vallée, and D. Côté, "Coherent anti-stokes raman scattering hyperspectral tissue imaging with a wavelength-swept system," Biomed. Opt. Express, vol. 2, pp. 1296-1306, May 2011.

[83] R. S. Lim, J. L. Suhalim, S. Miyazaki-Anzai, M. Miyazaki, M. Levi, E. O. Potma, and B. J. Tromberg, "Identification of cholesterol crystals in plaques of atherosclerotic mice using hyperspectral CARS imaging," Journal of Lipid Research, vol. 52, pp. 2177-2186, 2011.

[84] R. Boyd, Nonlinear Optics. Academic Press, 3rd ed., 2008.

[85] N. Bloembergen, Nonlinear Optics. World Scientific, 4 ed., 1964.

[86] C. L. Evans, E. O. Potma, , and X. S. Xie, "Coherent anti-Stokes Raman scattering spectral interferometry: determination of the real and imaginary components of nonlinear susceptibility $\chi^{(3)}$ for vibrational microscopy," Optics Letters, vol. 29, pp. 29232925, December 2004.

[87] J.-X. Cheng and X. S. Xie, "Coherent anti-Stokes Raman scattering microscopy: Instrumentation, theory, and applications," J. Phys. Chem. B, vol. 108, pp. 827-840, 2004 .

[88] O. Burkacky, A. Zumbusch, C. Brackmann, and A. Enejder, "Dual-pump coherent antistokes raman scattering microscopy," Optics Letters, vol. 31, pp. 3656-3658, December 
2006.

[89] A. Downes, R. Mouras, and A. Elfick, "A versatile cars microscope for biological imaging," J. Raman Spectrosc., vol. 40, no. 7, pp. 757-762, 2009.

[90] S. Brustlein, P. Ferrand, N. Walter, S. Brasselet, C. Billaudeau, D. Marguet, and H. Rigneault, "Optical parametric oscillator-based light source for coherent raman scattering microscopy: practical overview," Journal of Biomedical Optics, vol. 16, no. 2, pp. 021106-1-10, 2011.

[91] Y. Fu, H. Wang, R. Shi, and J.-X. Cheng, "Characterization of photodamage in coherent anti-stokes raman scattering microscopy," Optics Express, vol. 14, pp. 3942-3951, 2006.

[92] M. Balu, T. Baldacchini, J. Carter, T. Krasieva, R. Zadoyan, and B. Tromberg, "Effect of excitation wavelength on penetration depth in nonlinear optical microscopy of turbid media," Journal of Biomedical Optics, vol. 14, p. 010508, 2009.

[93] E. M. Vartiainen, H. A. Rinia, M. Müller, and M. Bonn, "Direct extraction of raman line-shapes from congested cars spectra," Opt. Express, vol. 14, pp. 3622-3630, Apr 2006.

[94] H. A. Rinia, M. Bonn, M. Mller, and E. M. Vartiainen, "Quantitative CARS spectroscopy using the maximum entropy method: The main lipid phase transition," Chem. Phys. Chem, vol. 8, no. 2, pp. 279-287, 2007.

[95] Y. Liu, Y. J. Lee, and M. T. Cicerone, "Broadband CARS spectral phase retrieval using a time-domain kramers-kronig transform," Opt. Lett., vol. 34, pp. 1363-1365, May 2009.

[96] C. Brandel, Y. Amharar, J. M. Rollinger, U. J. Griesser, Y. Cartigny, S. Petit, and G. Coquerel, "Impact of molecular flexibility on double polymorphism, solid solutions and chiral discrimination during crystallization of diprophylline enantiomers," Molecular Pharmaceutics, vol. 10, pp. 3850-3861, 2013.

[97] D. A. McQuarrie and J. D. Simon, Physical Chemistry: A Molecular Approach. University Science Books, 1997.

[98] R. W. Hellwarth, "Third-order optical susceptibilities of liquids and solids," Journal of Progress in Quantum Electronics, vol. 5, pp. 1-68, 1977.

[99] C. C. Shang and H. Hsu, "The spatial symmetric forms of third-order nonlinear susceptibility," IEEE Journal of Quantum Electronics, vol. 2, pp. 177-179, 1987.

[100] E. Da Silva, S. Bresson, and D. Rousseau, "Characterization of the three major polymorphic forms and liquid state of tristearin by Raman spectroscopy," Chem. Phys. Lipids, vol. 157, no. 2, pp. 113-119, 2009.

[101] L. Yu, N. Milton, E. G. Groleau, D. S. Mishra, and R. E. Vansickle, "Existence of a mannitol hydrate during freeze-drying and practical implications," Journal of Pharmaceutical Sciences, vol. 88, pp. 196-198, February 1999. 
[102] A. Burger, J. O. Henck, J. M. Hetz, S.and Rollinger, W. A. A., and H. Stttner, "Energy/temperature diagram and compression behavior of the polymorphs of Dmannitol.," Journal of Pharmaceutical Sciences, vol. 89, no. 4, pp. 457-468, 2000.

[103] L. Yu, "Amorphous pharmaceutical solids: preparation, characterization, and stabilization," Advanced Drug Delivery Reviews, vol. 48, pp. 27-42, 2001.

[104] Y. Xie, W. Cao, S. Krishnan, H. Lin, and N. Cauchon, "Characterization of mannitol polymorphic forms in lyophilized protein formulations using a multivariate curve resolution (MCR)-based Raman spectroscopic method," Pharmaceutical Research, vol. 25, pp. 2292-2301, October 2008.

[105] J. R. Beattie, L. J. Barrett, J. F. Malone, J. J. McGarvey, M. Nieuwenhuyzen, and V. L. Kett, "Investigation into the subambient behavior of aqueous mannitol solutions using temperature-controlled Raman microscopy," European Journal of Pharmaceutics and Biopharmaceutics, vol. 67, pp. 569-578, March 2007.

[106] L. Yu, "Nucleation of one polymorph by another," JACS Communications, vol. 125, no. 21, pp. 6380-6381, 2003.

[107] S. Ward, M. Perkins, J. Zhang, C. J. Roberts, C. E. Madden, S. Y. Luk, N. Patel, and S. J. Ebbens, "Identifying and mapping surface amorphous domains," Pharmaceutical Research, vol. 22, no. 7, pp. 1195-1202, 2005.

[108] D. J. Newman, G. M. Cragg, and K. M. Snader, "Natural products as sources of new drugs over the period 19812002," Journal of Natural Products, vol. 66, no. 7, pp. 1022$1037,2003$.

[109] M. S. Butler, "The role of natural product chemistry in drug discovery," Journal of Natural Products, vol. 67, no. 12, pp. 2141-2153, 2004.

[110] I. Paterson and E. A. Anderson, "The renaissance of natural products as drug candidates," Science, vol. 310, no. 5747, pp. 451-453, 2005.

[111] J. M. McPartland and E. B. Russo, "Cannabis and cannabis extracts: Greater than the sum of their parts?," Journal of Cannabis Therapeutics, vol. 1, no. 3/4, pp. 103-132, 2001.

[112] D. Baker, G. Pryce, G. Giovannoni, and A. J. Thompson, "The therapeutic potential of cannabis," The Lancet Neurology, vol. 2, no. 5, pp. 291-298, 2003.

[113] B. D. Backer, B. Debrus, P. Lebrun, L. Theunis, N. Dubois, L. Decock, A. Verstraete, P. Hubert, and C. Charlier, "Innovative development and validation of an HPLC/DAD method for the qualitative and quantitative determination of major cannabinoids in cannabis plant material," Journal of Chromatography B, vol. 877, pp. 4115-4124, 2009.

[114] M. A. ElSohly and D. Slade, "Chemical constituents of marijuana: The complex mixture of natural cannabinoids," Life Sciences, vol. 78, pp. 539-548, 2005.

[115] C. Brackmann, A. Bengtsson, M. L. Alminger, U. Svanberg, and A. Enejder, "Visualization of beta-carotene and starch granules in plant cells using CARS and SHG 
microscopy," Journal of Raman Spectroscopy, vol. 42, no. 4, pp. 586-592, 2011.

[116] J. C. Mansfield, G. R. Littlejohn, M. P. Seymour, R. J. Lind, S. Perfect, and J. Moger, "Label-free chemically specific imaging in planta with stimulated Raman scattering microscopy," Analytical Chemistry, vol. 85, pp. 5055-5063, April 2013.

[117] M. Malceva, M. Vikmane, and V. Stramkale, "Changes of photosynthesis-related parameters and productivity of cannabis sativa under different nitrogen supply," Environmental and Experimental Biology, vol. 9, pp. 61-69, 2011.

[118] Y. Zeng, Y. Wu, D. Li, W. Zheng, W.-X. Wang, and J. Y. Qu, "Two-photon excitation chlorophyll fluorescence lifetime imaging: a rapid and noninvasive method for in vivo assessment of cadmium toxicity in a marine diatom thalassiosira weissflogii," Planta, vol. 236, pp. 1653-1663, July 2012.

[119] S. Sirikantaramas, F. Taura, Y. Tanaka, Y. Ishikawa, S. Morimoto, and Y. Shoyama, "Tetrahydrocannabinolic acid synthase, the enzyme controlling marijuana psychoactivity, is secreted into the storage cavity of the glandular trichomes," Plant Cell Physiology, vol. 46, no. 9, pp. 1578-1582, 2005.

[120] Y. Liu, Y. J. Lee, and M. T. Cicerone, "Fast extraction of resonant vibrational response from CARS spectra with arbitrary nonresonant background," J. Raman Spectrosc., vol. 40, no. 7, pp. 726-731, 2009.

[121] A. Volkmer, J.-X. Cheng, and X. S. Xie, "Vibrational imaging with high sensitivity via epidetected coherent anti-Stokes Raman scattering microscopy," Phys. Rev. Lett., vol. 87, no. 2, pp. 023901-(1-4), 2001.

[122] A. Zare, Hyperspectral endmember detection and band selection using Bayesian methods. Phd thesis, University of Florida, 2008.

[123] A. Zare and P. Gader, "Spice: A sparsity promoting iterated constrained endmember extraction algorithm with applications to landmine detection from hyperspectral imagery," Proc. SPIE, vol. 6553, pp. 655319-1-9, 2007.

[124] Y. Rubner, C. Tomasi, and L. J. Guibas, "The Earth Movers Distance as a metric for image retrieval," International Journal of Computer Vision, vol. 40, no. 2, pp. 99-121, 2000.

[125] A. Zare and D. T. Anderson, "Earth Movers Distance-based simultaneous comparison of hyperspectral endmembers and proportions," IEEE Journal Of Selected Topics In Applied Earth Observations And Remote Sensing, vol. In press, pp. 1-12, 2014.

[126] W. N. Martens, R. L. Frost, J. Kristof, and J. T. Kloprogge, "Raman spectroscopy of dimethyl sulphoxide and deuterated dimethyl sulphoxide at 298 and $77 \mathrm{k}$," Journal of Raman Spectroscopy, vol. 33, pp. 84-91, 2002.

[127] S. Mukamel, Principles of Nonlinear Optical Spectroscopy. Oxford Universtiy Press, 1995.

[128] M. O. Scully and M. S. Zubairy, Quantum Optics. Cambridge University Press, 1997. 
[129] Y. R. Shen, The Principles of Nonlinear Optics. Wiley, 2002.

[130] S. Rahav, O. Roslyak, and S. Mukamel, "Manipulating stimulated coherent antiStokes Raman spectroscopy signals by broad-band and narrow-band pulses," Journal of Chemical Physics, vol. 131, pp. 194510-1-11, 2009.

[131] H. Du, R. A. Fuh, J. Li, A. Corkan, and J. S. Lindsey, "Photochemcad: A computeraided design and research tool in photochemistry," Photochemistry and Photobiology, vol. 68, pp. 141-142, 1998. 



\section{APPENDIX A}

\section{Noise in VPC-CARS measurements}

There are three primary sources of noise in a VPC-CARS setup: detector dark noise, electronic thermal noise, and optical shot noise. To readily compare these three noise sources we use a value called the noise equivalent power (NEP), which is a measure of the optical power incident on a detector system needed to generate a certain electrical signal. The NEP of a photodiode detector is normally given in terms of watts per root hertz; for the silicon photodiode we use the NEP is specified at $2 \times 10^{-13} \mathrm{~W} / \sqrt{\mathrm{Hz}}$ at $970 \mathrm{~nm}$ and $-5 \mathrm{~V}$ bias. For our experimental conditions, with $\lambda_{A S} \approx 810 \mathrm{~nm}$ and a $-60 \mathrm{~V}$ bias, the detector NEP is calculated to be

$$
\begin{aligned}
\mathrm{NEP} & =\sqrt{\left(2 q I_{D} \mathrm{BW}\right)+\left(\frac{4 k_{b} T}{R_{S H}}\right)} \\
& =7.3 \times 10^{-13} \mathrm{~W} / \sqrt{\mathrm{Hz}} .
\end{aligned}
$$

In this calculation, $q=1.6 \times 10^{-19} \mathrm{C}$ is the charge on the electron, $I_{D}$ is the detector dark current (in Amps) due to the bias voltage through the detector shunt resistance $R_{S H} \approx 5 \mathrm{G} \Omega, k_{b}=1.38 \times 10^{-23} \mathrm{~J} / \mathrm{K}$ is the Boltzmann constant, and $T$ is the temperature in Kelvin.

Shot noise is a statistical spread in the number of quanta measured from a source per unit time, and is given as the square root of the number of quanta detected. These quanta can be photons or electronics; the statistics are identical and given by a Poissonian distribution. The formula for electronic shot noise is simply the first term on the right-hand side of Eq. A.1, and forms the majority of the resulting NEP of the detector.

The other term in Eq. A.1 is the thermal Johnson-Nyquist current noise, which is a measure of the ability of electrons to move within a material, and is inversely proportional to the square root of the resistance $R_{S H}$ of the material and directly proportional to the temperature $T$ of the material and the measurement bandwidth BW. Photodiodes naturally have very high shunt re- 
sistances, and so generally have small thermal noise values.

Optical shot noise is proportional to the square root of the number of detected photons, $\sigma_{\text {phot }}=\sqrt{\left\langle N_{\text {phot }}\right\rangle}$. For a reasonable local oscillator power of $30 \mathrm{~mW}$ $\left(N_{\text {phot }}=1.2 \times 10^{17}\right.$ at $\left.810 \mathrm{~nm}\right)$, the shot noise power is

$$
\begin{aligned}
P_{\sigma} & =\sqrt{\left\langle N_{\text {phot }}\right\rangle} \cdots E_{\text {phot }} \\
& =8.5 \times 10^{-11} \mathrm{~W} .
\end{aligned}
$$

Optical shot noise is clearly dominant over intrinsic detector noise. The final source of noise is electronic noise, which is provided almost entirely by the initial coupling resistor after the photodiode and is purely thermal, following the same form

$$
\sigma_{V}=\sqrt{4 k_{B} T R}
$$

as given above for the photodiode thermal noise. Our system has a $50-\Omega$ termination resistor, which has a thermal voltage noise of $1.05 \mathrm{nV} / \sqrt{\mathrm{Hz}}$ that translates to a NEP of $3.3 \times 10^{-11} \mathrm{~W} / \sqrt{\mathrm{Hz}}$. This value is not very far off from the optical shot NEP.

To guarantee that low-bandwidth detection is shot-noise limited we perform noise measurements using the 50 - $\Omega$-terminated photodiode at a range of incident power levels. The theoretical NEP curves for five termination resistor values are shown as solid curves in Fig. A.1. The shot noise (pink dashed line) and individual thermal noise values (horizontal dotted lines) are also plotted. Noise measurements performed on the setup are compared with theory in Fig. A.1 for these five resistor values. Good agreement is found with the theory for a $50-\Omega$ termination resistor, but theory and experiment show that this setting is not ideal. Measurements conducted at this bandwidth are just barely in the shot-noise-limited window: at high optical powers the photodiode saturates, causing the drop in the NEP above $60 \mathrm{~mW}$, while on the low end the NEP is only a factor of two above the resistor noise level. While this power regime is technically shot-noise limited, the difference between the shot noise and the thermal noise is minimal. Switching to a $30-\mathrm{k} \Omega$ transimpedance resistor would be a marked improvement, offering at least one and a half orders of magnitude of increased optical dynamic range and placing measurements squarely in the shot-noise limited window. The $50-\Omega$ resistor value was chosen because it automatically forms an impedance-matched network with other RF electronic 


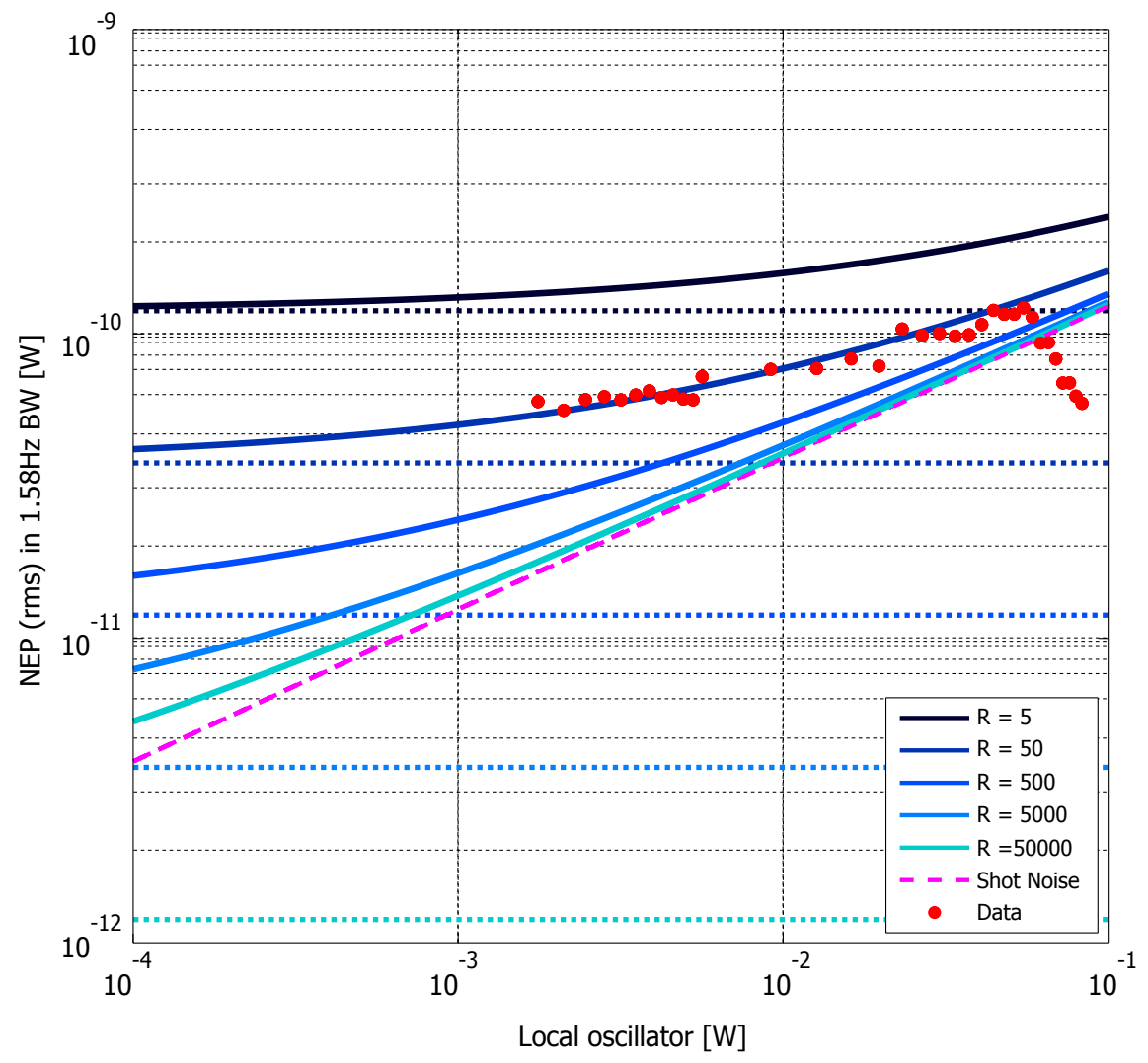

Figure A.1. Noise equivalent power curves for a range of termination resistor values as a function of incident optical power. The horizontal lines are the thermal NEP values for each resistor value, and the magenta diagonal line with slope $1 / 2$ is the shot noise. The red dots are measurements with a $50-\Omega$ termination resistor, which sit on the cusp between the shot-noise and thermal-noise regimes. The roll-off of the curve for values higher than $60 \mathrm{~mW}$ is due to optical saturation of the photodiode.

components that are further downrange in the signal chain. 



\section{Summary}

Throughout this thesis we have demonstrated methods for increasing the contrast of coherent Raman scattering experiments. In the most basic terms, CRS contrast is derived from the resonant motions of atoms relative to each other within molecules, but is fundamentally limited by a persistent non-resonant background. Removing, suppressing, or mitigating this background is the most straightforward path toward enhanced contrast. There are many techniques that have been developed to this end, and we have added three more to the CRS toolbox. The immediate application of two of these techniques has been the microscopic analysis and characterization of polymorphic materials, and we are confident that others will soon follow.

Chapter 2 is about the basic theoretical and experimental implementations of hyperspectral coherent anti-Stokes Raman scattering (CARS) in a spectrally narrowband setup. The thrust of this research was the creation of hyperspectral acquisition routines and analysis tools that enable the rapid, qualitative characterizations of samples in-line. We built a system that is capable of acquiring hyperspectral CARS data stacks in tens of seconds, then quickly processing the complicated three-dimensional data to yield a set of intuitive, two-dimensional images with high visual contrast. This system has become the workhorse of the Optical Sciences CARS lab - it is routinely used as a first-stage diagnostic tool for new samples - and has found use in a number of applications, including the in situ analysis of the constituent components of pharmaceutical oral dosage forms. The introduction of a modulation technique further enables the suppression of certain electronic background contributions, which allowed us to image the in planta distributions of the active compounds in Cannabis sativa $L$.

Chapter 3 covers vibrational phase contrast (VPC)-CARS. The basic theory and initial experimental implementations of VPC-CARS were carried out by Jurna et al., and this work expands on both. On the theory side, we have expanded the mathematical framework of coherent Raman scattering to describe 
molecular systems where multiple resonances in different regions of the vibrational spectrum are probed simultaneously. Computational work based on this new model showed that two resonances in different vibrational manifolds can either mutually enhance or suppress the total vibrational signal. Experimentally, we have implemented an entirely new hardware architecture to increase the imaging speed of the system by over two orders of magnitude compared to its predecessor, as well as added capabilities for imaging in epi-detection when detection in transmission is not possible. Further, the hyperspectral scanning capabilities of the previous section are applied to VPC-CARS. Complex vibrational spectra are collected at every point within a sample, enabling analysis beyond what is possible with data obtained from other methods. This analysis includes the first application of a quantitative endmember extraction algorithm called SPICE (Sparsity Promoting Iterative Contrained Endmember detection) on complex hyperspectral data. This algorithm attempts to determine the precise number of pure compounds that are present in a given sample based on a set of mixed data, as well as the spectra and relative proportions of those compounds within each pixel of the image. An initial analysis of the VPC-CARS data measured on quench-cooled mannitol, which is a highly complicated sample, has been very promising.

Chapter 4 introduces a new paradigm regarding CRS measurements. Building on the theoretical groundwork laid down by Rahav and Mukamel we have developed an experimental technique that casts heterodyne CARS in a fully quantum mechanical framework. The primary advantages of this new vibrational molecular interferometric (VMI) approach are twofold. First, by not singling out one beam in a heterodyne CARS experiment as the "local oscillator" from the beginning, all fields are treated equally and hence the analysis of the interaction is much simpler. Casting the optical interactions in terms of parametric and dissipative components further simplifies the interpretation of the data, and facilitates the direct extraction of the purely resonant component of the nonlinear susceptibility $\chi^{(3)}$. Second, although not explicitly predicted by Rahav and Mukamel, two-photon resonant electronic transitions can be measured simultaneously. With our setup we have measured the purely resonant component of a molecular vibration in the presence of a large electronic two-photon fluorescent background that overwhelms a standard CARS measurement.

The field of coherent Raman scattering microscopy has progressed rapidly over the past decade and a half, and the future looks bright. Challenges naturally still remain, in particular when it comes to interpreting CARS data. The 
non-resonant background intrinsically reduces contrast and generates artifacts that appear inexplicable to non-experts. While removing this background is ideal, the equipment required to do so is not trivial. The alternative that we propose is the qualitative representation of the spectral information as colors. We have found that training a user to interpret and understand the results of the spectral projection method takes less than an hour, and skilled users can parse data sets quicker than they can be acquired. Adding hyperspectral capabilities to more advanced techniques like VPC-CARS rapidly increases their utility across a broad field of applications, and entirely new paradigms allow the use of CRS methods on samples that have so far been considered inaccessible due to electronic backgrounds. 



\section{Samenvatting}

In dit proefschrift worden methodes gepresenteerd die het contrast van coherente Raman verstrooing (CRS) experimenten verbeteren. Eenvoudig gezegd komt CRS contrast voort uit resonante trillingen van atomen binnen moleculen. Dit contrast is echter fundamenteel gelimiteerd door een niet-resonant achtergrondsignaal. Het verwijderen, onderdrukken of verzachten van dit achtergrondsignaal is de meest directe weg naar een verbeterd contrast. Voor dit doel zijn al verschillende technieken ontwikkeld en wij hebben drie technieken toegevoegd. De directe toepassing van twee van deze technieken is te vinden in microscopische analyse en karakterisatie van polymorfe materialen. We verwachten dat andere toepassingen snel zullen volgen.

Hoofdstuk 2 behandelt de algemene theoretische en experimentele implementatie van hyperspectrale coherente anti-Stokes Raman verstrooing (CARS) in een smalbandige setup. De motivatie achter dit onderzoek was het creëren van hyperspectrale verzamelroutines en analysemethodes die snelle en kwalitatief hoogwaardige karakterisaties van samples in-line mogelijk maakt. Wij bouwden een systeem dat binnen tientallen seconden hyperspectrale CARS datastacks kan verzamelen en deze complexe driedimensionale data snel verwerkt tot een set van intuitieve, tweedimensionale figuren met hoog visueel contrast. Dit systeem is inmiddels het werkpaard van het Optical Sciences CARS lab - het wordt vaak als first-stage diagnostische methode voor nieuwe samples gebruikt - en wordt voor verschillende doeleinden ingezet, waaronder het in situ analyseren van de ingredienten van farmaceutische orale tabletten. Het toepassen van een modulatietechniek maakt verdere onderdrukking van elektronische achtergrondruis mogelijk, waardoor wij ook in planta verspreiding van de actieve ingredienten van Cannabis sativa L. konden visualiseren.

In hoofdstuk 3 wordt vibrationeel fase-contrast (VPC)-CARS besproken. De algemene theorie en oorspronkelijke experimentele implementaties zijn uitgevoerd door Jurna et al., en dit werk borduurt voort op beide aspecten. In het theoretische gebied hebben we het wiskundig model van coherente Raman 
verstrooing uitgebreid waardoor het nu ook moleculaire systemen beschrijft waar meerdere resonanties in verschillende regios van het vibrationele spectrum tegelijk gemeten worden. Rekenkundig werk, gebaseerd op dit nieuwe model, toonde dat twee resonanties in verschillende vibrationele regios het totale vibrationele signaal kunnen versterken of verzwakken. Experimenteel gezien hebben we een nieuwe hardware-architectuur geimplementeerd, waardoor de snelheid van het gehele systeem twee ordes van grootte verbeterde ten opzichte van zijn voorganger. Daarnaast kan het huidige systeem ook in de epi-richting meten wanneer transmissiemetingen niet mogelijk zijn. Ook is de mogelijkheid om hyperspectrale scans te maken toegepast op VPC-CARS. Complexe vibrationele spectra worden voor elk punt in een sample gemeten, waardoor analyse mogelijk wordt die met data verkregen uit andere methodes nog onmogelijk was. Hieronder valt de eerste toepassing van een kwantitatief extractiealgoritme genaamd SPICE (Sparsity Promoting Iterative Constrained Endmember detection) op complexe hyperspectrala datastacks. Dit algoritme tracht-gebaseerd op een set van gemixte data - het precieze aantal pure componenten in een gegeven sample te bepalen. Daarnaast zoekt het ook spectra en relatieve verhoudingen van deze componenten binnen elk pixel van de afbeelding. Een eerste analyse van VPC-CARS data van quench-cooled mannitol, wat een zeer complex sample is, was veelbelovend.

In hoofdstuk 4 wordt een nieuw paradigma met betrekking tot CRS-metingen gentroduceerd. Voortbordurend op de theoretische basis van Rahav en Mukamel, ontwikkelden wij een experimentele techniek die heterodyne CARS vanuit een volledig kwantummechanisch kader benadert. De voordelen van deze nieuwe vibrationele moleculaire interferometrische (VMI) aanpak zijn in tweevoud. Ten eerste, omdat er in het heterodyne CARS experiment geen beam al van begin af aan als "local oscillator" wordt gedefinieerd, worden alle velden gelijk behandeld waardoor de analyse van de interactie veel eenvoudiger wordt. Het beschouwen van de optische interacties in termen van parametrische en dissipatieve componenten vereenvoudigt het interpreteren van de data nog verder en maakt directe extractie van het puur resonante component van de niet-lineaire susceptibiliteit, $\chi^{(3)}$, mogelijk. Ten tweede, twee-foton resonante electronische transities kunnen tegelijk met de CARS-signalen gemeten worden, hoewel dit niet expliciet voorspeld was door Rahav en Mukamel. Met onze setup konden we het puur resonante component van een moleculaire vibratie meten in aanwezigheid van een sterke elektronische twee-foton fluorescente achtergrond die een gewone CARS-meting zou overstemmen.

Het onderzoeksveld van coherente Raman verstrooing microscopie heeft de 
afgelopen anderhalve decennia grote stappen vooruit gezet en gaat een mooie toekomst tegemoet. Desondanks blijven er uitdagingen, met name bij het interpreteren van CARS-data. De niet-resonante achtergrond vermindert het contrast en veroorzaakt artefacten die voor veel niet-experts onverklaarbaar lijken. Hoewel het verwijderen van deze achtergrond ideaal zou zijn, is de benodigde apparatuur niet triviaal. Het alternatief dat wij voorstellen is het kwalitatief representeren van spectrale informatie in kleuren. Wij ontdekten dat het instrueren van een nieuwe gebruiker om de resultaten van deze spectrale projectiemethode te begrijpen niet meer dan een uur kost. Ervaren gebruikers bleken datasets zelfs sneller te kunnen verwerken dan dat ze verkregen kunnen worden. Het toevoegen van hyperspectrale technieken aan verder gevorderde methodes als VPC-CARS verbetert hun bruikbaarheid over een groot gebied van toepassingen, en volledig nieuwe paradigmas laten het gebruik van CRS methodes toe op samples die tot nu toe als ontoegankelijk werden beschouwd door de elektronische achtergronden. 



\section{APPENDIX D}

\section{List of publications}

\section{Articles covered in this thesis}

- E. T. Garbacik, R. P. Korai, E. H. Frater, J. P. Korterik, C. Otto, H. L. Offerhaus. "In planta imaging of $\Delta^{9}$-tetrahydrocannabinolic acid in Cannabis sativa L. with hyperspectral coherent anti-Stokes Raman scattering microscopy". Journal of Biomedical Optics 18 (4), pp. 046009 (2013).

- E. T. Garbacik, J. L. Herek, C. Otto, H. L. Offerhaus. "Rapid identification of heterogeneous mixture components with hyperspectral coherent anti- Stokes Raman scattering imaging". Journal of Raman Spectroscopy 43 (5), pp. 651-655 (2012).

- E. T. Garbacik, J. P. Korterik, C. Otto, S. Mukamel, J. L. Herek, H. L. Offerhaus. "Background-Free Nonlinear Microspectroscopy with Vibrational Molecular Interferometry" . Physical Review Letters 107, pp. 253902 (2011).

\section{Other peer-reviewed publications}

- A. L. Fussell, E. T. Garbacik, P. Kleinebudde, C. H. Strachan, H. L. Offerhaus. "In situ dissolution analysis using coherent anti-Stokes Raman scattering CARS and hyperspectral CARS microscopy". European Journal of Pharmaceutics and Biopharmaceutics 85 (3B), pp. 1141-1147 (2013).

- M. Arjaans, Th. H. O. Munnink, S. F. Oosting, A. G. T. Terwisscha van Scheltinga, J. A. Gietema, E. T. Garbacik, H. Timmer-Bosscha, M. N. Lub-de Hooge, C. P. Schroder, E. G. E. de Vries. "Bevacizumabinduced normalization of blood vessels in tumors hampers antibody uptake". Cancer Research 73 (11), pp. 3347-3355 (2013).

- N. M. Perney, L. Braddick, M. Jurna, E. T. Garbacik, H. L. Offerhaus, L. C. Serpell, E. Blanch, L. Holden-Dye, W. S. Brocklesby, T. Melvin. 
"Polyglutamine Aggregate Structure In Vitro and In Vivo; New Avenues for Coherent Anti-Stokes Raman Scattering Microscopy". PLoS ONE 7 (7), pp. e40536 (2012).

- H. L. Offerhaus, E. T. Garbacik, A. C. W. van Rhijn, A. L. Fussell, J. L. Herek. "Phase aspects of (broadband) stimulated Raman scattering". Reviews in Analytical Chemistry 31 (1), pp. 1-6 (2012).

- M. Jurna, E .T. Garbacik, J. P. Korterik, J .L. Herek, C. Otto, H. L. Offerhaus. "Visualizing Resonances in the Complex Plane with Vibrational Phase Contrast Coherent Anti-Stokes Raman Scattering". Analytical Chemistry 82 (16), pp. 7656-7659 (2010).

- K. Orsel, E. T. Garbacik, M. Jurna, J. P. Korterik, C. Otto, J. L. Herek and H. L. Offerhaus. "Heterodyne interferometric polarization coherent anti-Stokes Raman scattering (HIP-CARS) spectroscopy". Journal of Raman Spectroscopy 41 (12), pp. 1678-1681 (2010).

- P. F. Chimento, M. Jurna, H. S. P. Bouwmans, E. T. Garbacik, L. Hartsuiker, C. Otto, J. L. Herek, H. L. Offerhaus. "High-resolution narrowband CARS spectroscopy in the spectral fingerprint region". Journal of Raman Spectroscopy 40 (9), pp. 1229-1233 (2009).

\section{Conference proceedings}

- A. L. Fussell, E. T. Garbacik, K. Löbmann, H. L. Offerhaus, P. Kleinebudde, C. J. Strachan. "In situ dissolution analysis of pharmaceutical dosage forms using coherent anti-Stokes Raman scattering (CARS) microscopy". SPIE Proceedings 8948, pp. 8948J2 (2014).

- E. T. Garbacik, A. L. Fussell, S. Gres, J. P. Korterik, C. Otto, J. L. Herek, H. L. Offerhaus. "Hyperspectral coherent anti-Stokes Raman scattering microscopy for in situ analysis of solid-state crystal polymorph". SPIE Proceedings 8588, pp. 85880-1 (2013).

- Erik T. Garbacik, Jeroen P. Korterik, Cees Otto, Shaul Mukamel, Jennifer L. Herek, and Herman L. Offerhaus. "Background-free nonlinear microspectroscopy with vibrational molecular interferometry". SPIE Proceedings 8226, pp. 822605 (2012). 
- M. Jurna, E.T. Garbacik, J.P. Korterik, C. Otto, J.L. Herek, H.L. Offerhaus. "Vibrational phase contrast CARS microscopy for quantitative analysis". SPIE Proceedings 7569, pp. 75690F (2010).

\section{Articles in preparation}

- E. T. Garbacik, J. P. Korterik, J. L. Herek, H. L. Offerhaus. "Epidetection of vibrational phase contrast coherent anti-Stokes Raman scattering".

- E. T. Garbacik, J. P. Korterik, J. L. Herek, H. L. Offerhaus. "Selfhomodyne enhancement of vibrational resonances in multiple interfering manifolds".

- E. T. Garbacik, J. P. Korterik, A. Zare, H. L. Offerhaus. "Spectral unmixing of heterodyne CARS microscopy in the complex plane".

- A. Zare, E. T. Garbacik, H. L. Offerhaus. "Sparsity-promiting iterative constrained endmember identification using complex hyperspectral data."

\section{Invited oral presentations}

- Hyperspectral coherent anti-Stokes Raman scattering microscopy for in situ analysis of solid-state crystal polymorphs, presented at Chemistry department colloquium, University of California, Irvine, CA, USA; 8 February 2013.

- Supercharging nonlinear microscopy: enhancement of CARS via surface plasmon resonances, presented at Physics department colloquium, University of Exeter, Exeter, United Kingdom; 18 May 2012.

- Quantum CARS: beating (all) the background, presented at Quantum Optics group colloquium, University of Leiden, Leiden, the Netherlands; 13 January 2012.

- Quantum CARS: beating (all) the background, presented at MESA+ colloquium, University of Twente, Enschede, the Netherlands; 10 January 2012.

- Vibrational molecular interferometry, presented at ICAVS6, Rohnert Park, CA, USA; 15 June 2011. 


\section{Submitted oral presentations}

- "Spectral unmixing of heterodyne CARS microscopy in the complex plane", to be presented at Focus on Microscopy 2014, Sydney, Australia; 15 April 2014.

- Hyperspectral coherent anti-Stokes Raman scattering microscopy for in situ analysis of solid-state crystal polymorphs, presented at ECONOS 2013, Exeter, United Kingdom; 24 April 2013.

- Hyperspectral coherent anti-Stokes Raman scattering microscopy for in situ analysis of solid-state crystal polymorphs, presented at SPIE Photonics West, BiOS section, San Francisco, CA, USA; 2 February 2013.

- Epi-detection of vibrational phase contrast CARS, presented at Applied Nanophotonics colloquium, University of Twente, Enschede, the Netherlands; 10 January 2013.

- Epi-detection of vibrational phase contrast CARS, presented at microCARS2012, Naurod, Germany; 16 October 2012.

- Rapid identification of heterogeneous mixture components with hyperspectral CARS microscopy, presented at the workshop on Advanced Coherent Raman Scattering (ACRS), University of Exeter, Exeter, United Kingdom; 24 April 2012.

- Phase aspects of coherent Raman interactions, presented at a joint colloquium with the University of Erlangen, Germany; 28 October 2011.

- Background-free nonlinear microspectroscopy with vibrational molecular interferometry, presented at the 35th annual NNV-AMO meeting, Lunteren, the Netherlands; 12 October 2011.

- Vibrational molecular interferometry, presented at ECONOS 2011, Enschede, the Netherlands; 25 May 2011.

- The molecular rollercoaster: its not just a phase, presented at the University of Twente annual MESA+ day, Enschede, the Netherlands; 14 September 2010.

- The molecular rollercoaster through complex space, presented at ECONOS 2010 conference, Bremen, Germany; 21 June 2010. 
- Riding the molecular rollercoaster through complex space, presented at Applied Nanophotonics colloquium, University of Twente, Enschede, the Netherlands; 1 April 2010.

- Heterodyne Interferometric Polarization Coherent Anti-Stokes Raman Scattering, presented at MicroCARS workshop in Cargse, Corsica, France; 8 October 2009 .

- Heterodyne Interferometric Polarization-Sensitive Coherent Anti-Stokes Raman Scattering, presented at 26th International School of Atomic and Molecular Spectroscopy in Erice, Sicily, Italy; 11 July 2009.

\section{Prizes and awards}

- EU/ESF COST grant for a Short-term Scientific Mission (STSM) to Exeter University, Exeter, UK (MP1102, coherent Raman scattering/MicroCoR); April/May 2012.

- Poster prize, awarded for "Vibrational molecular interferometry" at ICAVS6, Rohnert Park, CA, USA; 12 June 2011.

- Poster prize, awarded for "Monitoring dynamic processes in the complex plane with vibrational phase contrast CARS (with integrated video)" at the NWO Chemistry meeting, Veldhoven, The Netherlands; 18 January 2011.

- Het Beste Idee design contest winner, University of Twente; March 2010.

- Poster prize, awarded for "Vibrational phase contrast CARS" at the Workshop for Advanced Spectroscopy on Complex Systems, Ameland, The Netherlands; 8 June 2010. 
